Aus dem Institut für Allgemeinmedizin

(Prof. Dr. med. E. Hummers-Pradier)

der Medizinischen Fakultät der Universität Göttingen

\title{
Die medizinische Betreuung von Pflegeheimbewohnern
}

Perspektiven und Bedürfnisse von Angehörigen

\author{
INAUGURAL - DISSERTATION \\ zur Erlangung des Doktorgrades \\ der Medizinischen Fakultät der \\ Georg-August-Universität zu Göttingen
}

\author{
vorgelegt von \\ Juliane Langen \\ aus \\ Hennigsdorf
}

Göttingen 2016 
Dekan:

Referentin:

Ko-Referent:

Promotor-Vertretung:

Tag der mündlichen Prüfung: $\quad$ 30. November 2016
Prof. Dr. rer. nat. H. K. Kroemer

Prof. Dr. med. E. Hummers-Pradier

PD Dr. phil. M. Schweda

Prof. Dr. hum. biol. M. Schön 


\section{Inhaltsverzeichnis}

Inhaltsverzeichnis

Abbildungsverzeichnis ......................................................................II

Tabellenverzeichnis .....................................................................................II

$1 \quad$ Einleitung.........................................................................................

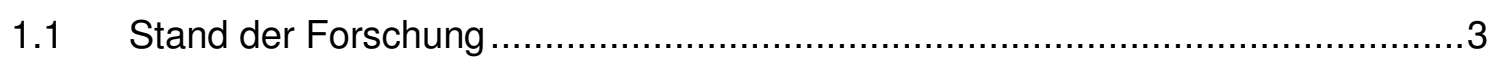

1.1.1 Forschungsstand im deutschsprachigen Raum ..........................................

1.1.2 Medizinische Versorgungstrukturen in deutschen Pflegeheimen .........................3

1.1.3 Perspektiven und Bedürfnisse der Angehörigen hinsichtlich der medizinischen

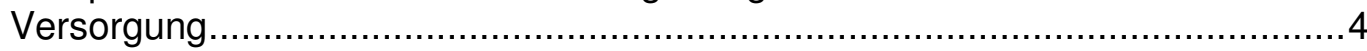

1.1.4 Perspektiven der Angehörigen hinsichtlich ihrer Aufgaben .............................6

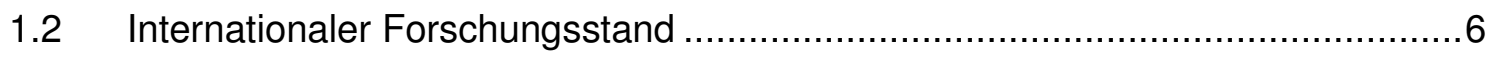

1.2.1 Perspektiven und Bedürfnisse der Angehörigen hinsichtlich der medizinischen Versorgung...........................................................................................

1.2.2 Perspektiven der Angehörigen hinsichtlich ihrer Aufgaben .............................. 10

1.2.3 Stellvertreterfähigkeit der Bezugspersonen für Bewohnerperspektiven ............11

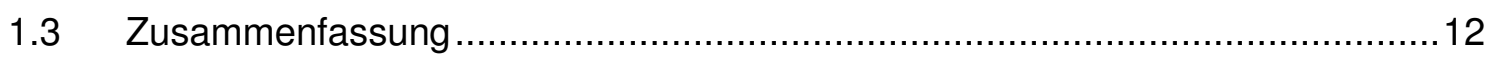

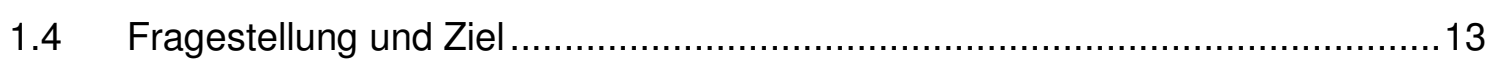

2 Material und Methoden .................................................................15

2.1 Methodische Anlage der Untersuchung und Einbettung in die Studie

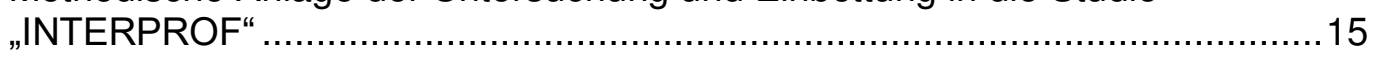

2.1.1 Begründung des qualitativen Vorgehens .................................................16

2.2 Stichprobe, Auswahlverfahren und Rekrutierung der Teilnehmer .....................17

2.3 Die Erhebungsmethode ..................................................................... 18

2.3.1 Das offene Leitfadeninterview ............................................................. 18

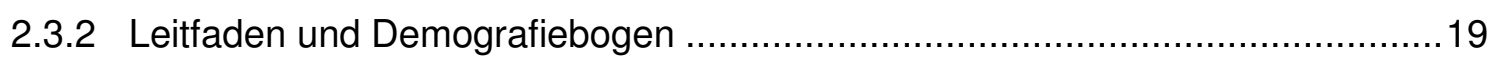

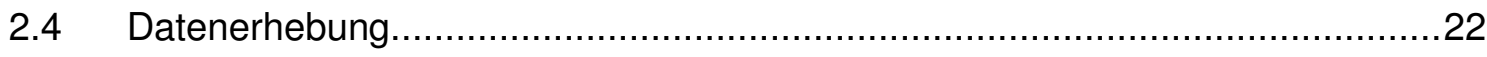

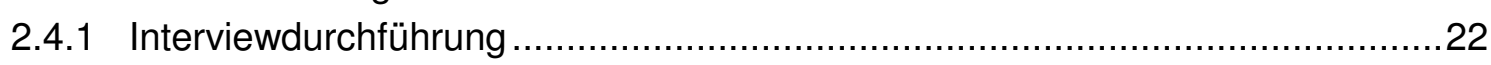

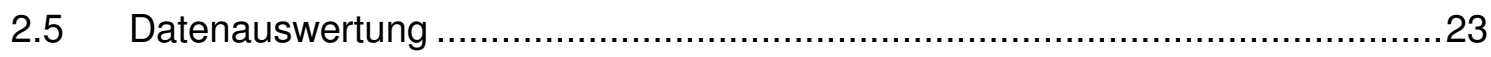

2.5.1 Die Grounded Theory Methode ...............................................................23

2.5.2 Anwendung der Grounded Theory Methode ............................................24

2.6 Computergestützte qualitative Datenanalyse...............................................28

2.7 Datenschutz und Genehmigung durch die Ethik-Kommission........................29

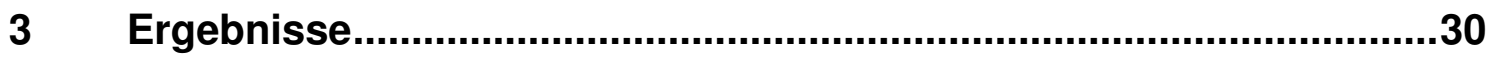

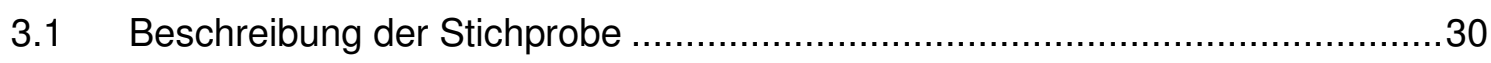

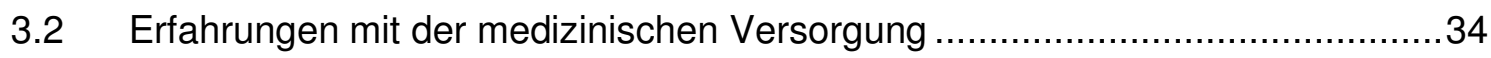

3.2.1 Arztbesuch im Pflegeheim ................................................................ 34 
3.2.2 Sichtweisen und Erfahrungen bezüglich der Ärzte.......................................40

3.2.3 Kontinuität in der medizinischen Versorgung ............................................ 52

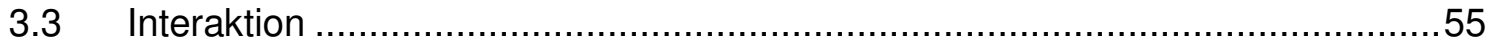

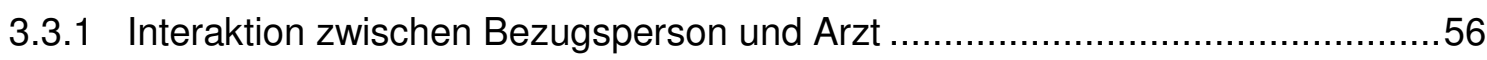

3.3.2 Interaktion zwischen Bezugsperson und Pflegekraft.....................................62

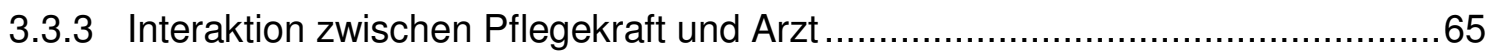

3.4 Aufgaben der Angehörigen/ Einbezogensein in die medizinische Versorgung..71

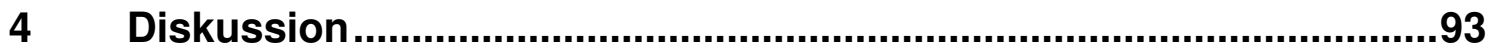

$4.1 \quad$ Stärken und Schwächen................................................................. 93

4.2 Einordnung in die themenbezogene Literatur ............................................ 95

4.3 Schlussfolgerungen und Ausblick ...................................................... 101

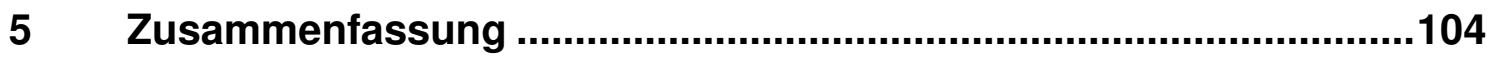

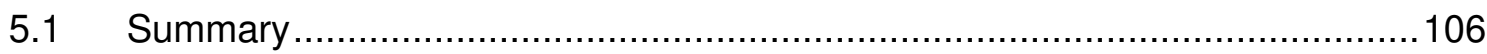

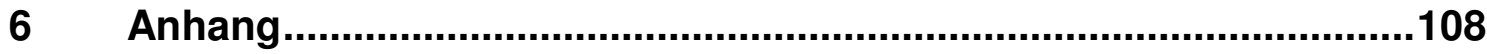

6.1 Anlage 1: Information für Bezugspersonen zur Teilnahme an der Studie.........108

6.2 Anlage 2: Einverständniserklärung zur Teilnahme an der Studie....................109

6.3 Anlage 3: Demografiebogen .......................................................... 110

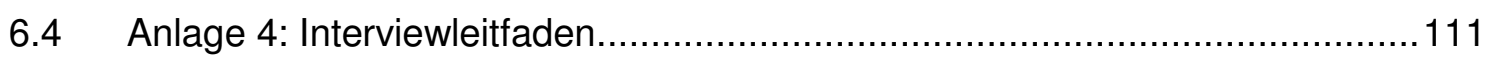

$7 \quad$ Literaturverzeichnis ..........................................................................113

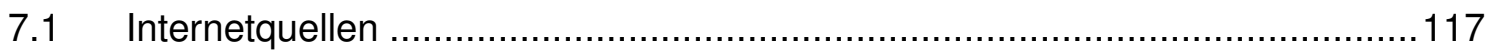




\section{Abbildungsverzeichnis}

Abb. 1 Codierparadigma nach Strauss und Corbin (Böhm 2012, S.479), die Verwendung der Abbildung erfolgt mit freundlicher Genehmigung des

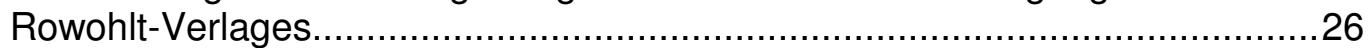

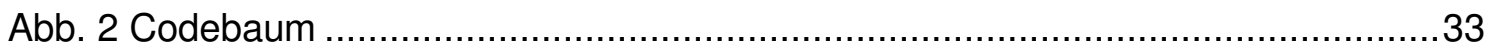

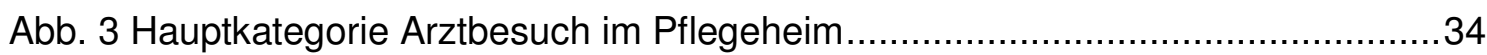

Abb. 4 Hauptkategorie Sichtweisen und Erfahrungen bezüglich der Ärzte .................41

Abb. 5 Hauptkategorie Kontinuität in der medizinischen Versorgung..........................52

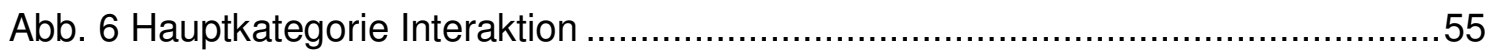

Abb. 7 Kategorie Interaktion zwischen Bezugsperson und Arzt ..................................56

Abb. 8 Kategorie Interaktion zwischen Bezugsperson und Pflegekraft .......................63

Abb. 9 Kategorie Interaktion zwischen Pflegekraft und Arzt...................................66

Abb. 10 Codierparadigma nach Strauss und Corbin (Böhm 2012, S.479), die

Verwendung der Abbildung erfolgt mit freundlicher Genehmigung des

Rowohlt-Verlages, Einbezogensein in die medizinische Versorgung................72

Abb. 11 Kontext, Einbezogensein in die medizinische Versorgung.............................73

Abb. 12 ursächliche Bedingungen, Einbezogensein in die medizinische Versorgung ..76

Abb. 13 intervenierende Bedingungen, Einbezogensein in die medizinische

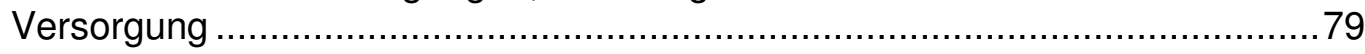

Abb. 14 Handlungsstrategien, Einbezogensein in die medizinische Versorgung .........83

Abb. 15 Konsequenzen, Einbezogensein in die medizinische Versorgung ..................89

\section{Tabellenverzeichnis}

Tabelle 1 Soziodemografische Angaben der befragten Angehörigen 


\section{Einleitung}

Die Vorhersagen sind deutlich die deutsche Bevölkerung schrumpft und die Gesellschaft vergreist. Dieses Älterwerden der Allgemeinheit bedingt, dass es immer mehr Menschen gibt, die im Alter versorgungsbedürftig werden. So waren 2,6 Millionen Menschen in Deutschland laut Statistischem Bundesamt zum Jahresende 2013 pflegebedürftig. Davon wurden gut zwei Drittel zu Hause und etwa 764.000 Menschen in vollstationärer Pflege versorgt. Wenn man ältere Versorgungszahlen mit denen von heute vergleicht, wird sowohl ein demografischer als auch ein gesellschaftlicher Wandel offenkundig. So hat sich seit 1999 die Zahl der in Pflegeheimen vollstationär Betreuten um rund 36 \% erhöht (Statistisches Bundesamt 2015). Zudem wird beobachtet, dass Pflegebedürftige zunehmend im Pflegeheim oder durch ambulante Pflegedienste und nicht durch ihre Familien versorgt werden. Möglicherweise ist dies durch den Rückgang der Möglichkeiten der familiären Pflege bedingt (statistische Ämter des Bundes und der Länder 2010). Mit der immer älter werdenden Bevölkerung in Deutschland wird geschätzt, dass die Zahl der Pflegebedürftigen bis 2030 auf circa 3,37 Millionen steigt (statistische Ämter des Bundes und der Länder 2010). Da aktuell bereits zahlreiche Menschen in der stationären Altenpflege leben und die Anzahl der Betroffenen weiter steigen wird, erlangt die Versorgung unserer alternden Bevölkerung in diesem Setting zunehmende Wichtigkeit. Um eine gute Betreuung sicherzustellen, ist es von großer Bedeutung, die Perspektiven und Bedürfnisse der betroffenen Akteure im Pflegeheim zu erfassen und etwaige Mängel in der Versorgung in Erfahrung zu bringen. Die vorliegende Arbeit wird dabei vor allem die medizinische Versorgung in den Fokus stellen, da durch die steigende Zahl der Pflegeheimbewohner, die in der Regel multimorbide mit hohem Behandlungsbedarf sind (van den Busche et al. 2009a), hier neue Herausforderungen entstehen.

Damit die Versorgung im Pflegeheim von allen Seiten beleuchtet werden kann, erscheint es neben der Untersuchung der Bedürfnisse der Bewohner ${ }^{1}$ selbst sinnvoll, die Perspektiven und Wünsche der Angehörigen bzw. privaten

\footnotetext{
${ }^{1}$ In der vorliegenden Arbeit wird durchgehend aus Gründen der besseren Lesbarkeit nur die maskuline Form verwendet, auch wenn sich auf beide Geschlechter bezogen wird.
} 
Bezugspersonen der Bewohner zu kennen. Befragungen zur Versorgung bei den Bewohnern, beispielsweise durch den Medizinischen Dienst des Spitzenverbandes Bund der Krankenkassen ergaben, dass die Pflegeheimbewohner stets sehr gute Bewertungen vergaben und kaum Kritik äußerten (Medizinischen Dienst des Spitzenverbandes Bund der Krankenkassen 2012). Als Grund wurde hier aus der Literatur vor allem das Abhängigkeitsverhältnis der Bewohner von der zu bewertenden Einrichtung angeführt (Hasseler und Wolf-Ostermann 2010; Wingenfeld 2003). Differenziertere und individuellere Meinungen lassen jedoch offene Befragungen von Bewohnern und auch Angehörigen erwarten. Zwar wird häufig behauptet, dass den Bezugspersonen die nötige Fachkenntnis für Bewertungen von Leistungen im Pflegeheim fehle (Nübling et al. 2004b). Nichtsdestotrotz haben Angehörige "durch ihre häufigen Besuche meist einen differenzierten Gesamteindruck von einer Einrichtung und der dort umgesetzten Versorgungsqualität" (Nübling et al. 2004b, S.138).

Ziel dieser Arbeit ist es, die Ansichten, Vorstellungen und Wünsche der privaten Bezugspersonen darzustellen. Die Ergebnisse ergänzen andere Studieninhalte (siehe Kap. 4.1), die sich mit den Standpunkten von Bewohnern, Ärzten und Pflegekräften auseinandersetzen, um so zu einem Gesamtbild der Sichtweisen der Akteure im Pflegeheim zu gelangen. 


\subsection{Stand der Forschung}

\subsubsection{Forschungsstand im deutschsprachigen Raum}

In Deutschland gibt es wenig Literatur zu Befragungen von privaten Bezugspersonen in der stationären Altenpflege hinsichtlich der medizinischen Versorgung der Bewohner. Im Verlauf der Literaturrecherche konnten zwar einige in Deutschland durchgeführte Angehörigenbefragungen im Pflegeheim ermittelt werden, jedoch ohne Informationsgewinn speziell zur medizinischen Versorgung (Born und Weigel 2000, Busch et al. 2006, Engels und Pfeuffer 2009, Mutscher et al. 2000, Kriz et al. 2006). Insgesamt ist festzustellen, dass den recherchierten Studien mehrheitlich ein quantitatives Design zu Grunde liegt.

\subsubsection{Medizinische Versorgungstrukturen in deutschen Pflegeheimen}

Multimorbiditätsbedingt bereitet es vielen Pflegeheimbewohnern Schwierigkeit ambulante Versorgungsmöglichkeiten in Anspruch zu nehmen. So wird häufig anstatt des Praxisbesuchs eher der Kontakt mit dem Arzt im Pflegeheim zur Regel (Balzer et al. 2013). In diesem Zusammenhang reduziert sich auch die selbstständige Initialisierung der Arztkontakte stark. So gaben in einer Fragebogenerhebung in 782 deutschen Heimen Pflegedienstleitungen etwa an, dass nahezu alle Arztkontakte meistens (62\%) oder sogar immer (37\%) vom Pflegepersonal veranlasst werden. Wiederum würden rund $80 \%$ der Bewohner und auch der Angehörigen dies selten selbst übernehmen (Hallauer et al. 2005). Mit zunehmender Pflegebedürftigkeit steigt auch der Anteil derjenigen, die eine gesetzliche Betreuung erhalten. Demnach leben mehr als die Hälfte aller gesetzlich betreuten Personen in stationären Einrichtungen (Internetredaktion des Bundesministeriums für Familie, Senioren, Frauen und Jugend 2006). Aus einer Aktenstichprobe $(n=201)$ in verschiedenen Standorten entnehmen Hoffmann und Korte zudem, dass in 62-74 \% der Fälle die Betreuung von Familienmitgliedern übernommen wird und dies bei rund einem Drittel auch den Aufgabenkreis der Gesundheitsfürsorge einschließt (Hoffmann und Korte 2002).

Bezüglich medizinischer Versorgungstrukturen in Pflegeheimen spielt der Hausarzt eine zentrale Rolle. Aus einer systematischen Literaturübersicht von 
Balzer et al. 2013 geht hervor, dass die ärztliche Versorgung in den Pflegeheimen vor allem von Allgemeinmedizinern geleistet wird. So haben die Heimbewohner laut einem GEK-Report annähernd einen Behandlungsfall beim Hausarzt pro Quartal (Rothgang et al. 2008). In einer anderen Erhebung (4.481 Bewohner in 58 Pflegeheimen) hatten circa $90 \%$ der stationär versorgten Pflegebedürttigen in den letzten vier Wochen einen persönlichen Hausarztkontakt (Schäufele et al. 2009). Angaben zur Organisation der medizinischen Versorgung wurden in einer MuG-IV Teilstudie erhoben. In Deutschland herrscht das Prinzip der freien Arztwahl. Dennoch verfügte etwa ein Fünftel der Heime $(n=609)$ über feste Vereinbarungen mit niedergelassenen Ärzten. Fest angestellte Ärzte (1\%) und Arztpraxen innerhalb einer Einrichtung waren eher selten (3\%) (Schneekloth und von Törne 2009).

Auch mögliche Problembereiche innerhalb der medizinischen Versorgung in deutschen Pflegeeinrichtungen führen Balzer et al. 2013 in ihrer Literaturübersicht auf. Die Autoren weisen jedoch darauf hin, dass hierbei ebenso kleinere Studien Berücksichtigung fanden und diese Zusammenstellung nur als Hinweis auf mögliche Probleme zu betrachten ist. Dabei werden folgende Bereiche beschrieben:

- Unzureichende Kommunikation zwischen Haus- und Fachärzten

- Nicht vorhandene, missverständliche oder fachlich unangemessene ärztliche Anordnungen

- Nicht angemessene Umsetzung ärztlicher Verordnungen durch Pflegende

- Ungenügend genaue ärztliche Untersuchung und Dokumentation diagnostischer Befunde (insbesondere im neuropsychiatrischen Bereich)

- Mangelhafte Dokumentation gesundheitlicher Beschwerden und medizinischpflegerischer Handlungen durch Pflegende

\subsubsection{Perspektiven und Bedürfnisse der Angehörigen hinsichtlich der medizinischen Versorgung}

In den letzten Jahren wurden verschiedene Studien durchgeführt, die sich unter anderem mit den Perspektiven von Angehörigen, deren Zufriedenheit und 
Wünschen hinsichtlich der medizinischen Versorgung ihrer im Pflegeheim wohnenden Angehörigen beschäftigten.

So zeigte sich in einer Fragebogenerhebung mit offenem Frageteil, dass von den 68 Angehörigen von Bewohnern eines deutschen Altenheims nicht einmal ein Drittel zufrieden mit der ärztlichen Versorgung der Bewohner war. Die Autoren führen die schlechte Bewertung der medizinischen Versorgung in diesem speziellen Fall auch auf die Vielzahl der dort tätigen Ärzte zurück, da diese aufgrund geringer Patientenzahlen im Heim nur selten Besuche machen würden. Im offenen Fragenteil wünschten sich die Befragten, unter anderem besser über Änderungen der Medikation oder geplante Visiten informiert zu werden (Bemmann und Klewer 2012).

In einer qualitativen Studie stellte sich jedoch heraus, dass private Bezugspersonen kognitiv nicht beeinträchtigter Bewohner nur einen groben Überblick über die Medikation der Bewohner hatten und sich selten bemühten, darüber mit deren Hausarzt zu sprechen. Es wurde festgestellt, dass die persönlichen Bezugspersonen den Hausarzt ihres Angehörigen in den meisten Fällen höchstens namentlich kannten und keine Übersicht über andere behandelnden Fachärzte hatten. Die interviewten Angehörigen hatten generell selten und dann eher zu Beginn des Heimaufenthaltes des Bewohners Kontakt mit den Ärzten. Eine Zusammenarbeit unter den verschiedenen Ärzten wurde oftmals fälschlicher Weise vorausgesetzt (Struppek 2010). Die Kommunikation zwischen Haus- und Fachärzten ist jedoch, wie bereits in Kapitel 2.1.1 beschrieben, gemäß einer Literaturübersicht von Balzer et al. 2013, einer der aufgeführten Problembereiche in der medizinischen Versorgung in deutschen Pflegeheimen. Zudem gingen die privaten Bezugspersonen häufig davon aus, dass Bewohner Haus- und Facharztbesuche initialisieren können (Struppek 2010). Aus einer anderen quantitativen Studie geht allerdings eher, wie bereits in Kapitel 2.1.1 beschrieben, hervor, dass die Initialisierung in der Regel von den Pflegekräften im Heim vorgenommen wird (Hallauer et al. 2005).

Über die Möglichkeit, jeder Zeit einen Arzt zu rufen, wurden 1489 Angehörige von Heimbewohnern auch in einer quantitativen Studie von Nübling et al. befragt. Außerdem sollten die privaten Bezugspersonen die Qualität, die Erreichbarkeit sowie die Möglichkeit der freien Arztwahl auf einer Skala von $1=$ sehr gut bis $5=$ 
sehr schlecht einschätzen. Die ärztliche Versorgung wurde insgesamt hinsichtlich der vier Einzelaspekte als gut $(2,3)$ beurteilt, wobei die Bewertungen der einzelnen Items nicht veröffentlich wurden (Nübling et al. 2004a).

\subsubsection{Perspektiven der Angehörigen hinsichtlich ihrer Aufgaben}

Der überwiegende Teil der Besuche von Angehörigen im Pflegeheim wird durch die Kinder der Bewohner durchgeführt. Von diesen kommen etwa zwei Drittel einoder mehrmals pro Woche ins Heim. Dies geht aus Angehörigenbefragungen einer Studie im Auftrag des Bundesministeriums für Familie, Senioren, Frauen und Jugend hervor (Engels und Pfeuffer 2009). Was die Formen der Mitwirkung betrifft, so beteiligen sich fast alle befragten privaten Bezugspersonen an der psychosozialen Betreuung (Unterhalten mit dem Bewohner, Vorlesen). Ein Großteil ist auch in die Regelung finanzieller Angelegenheiten einbezogen und erledigt kleinere Besorgungen $a b$ und $z u$ bis regelmäßig. Aktiv an der hauswirtschaftlichen Hilfe oder Pflege beteiligen sich hingegen nur $14 \%$ der Angehörigen. Mehr als die Hälfte der privaten Bezugspersonen vertraut auf eine gute Versorgungsqualität im Pflegeheim, diese wird aber auch von $21 \%$ selbst kontrolliert. Insgesamt ist festzustellen, dass die Angehörigen ein bedeutendes Bindeglied zwischen den früheren und den augenblicklichen Lebensbereichen der Bewohner sind (Engels und Pfeuffer 2009), weshalb es wichtig erscheint, deren Erfahrungen und Perspektiven im Hinblick auf die pflegerische, aber auch medizinische Versorgung im Pflegeheim zu erheben.

\subsection{Internationaler Forschungsstand}

International findet sich mehr Literatur zum Thema Angehörigenbefragungen im Pflegeheim. Diese sind aufgrund der sehr unterschiedlichen Versorgungssysteme jedoch nur eingeschränkt vergleichbar. Ein Großteil der Studien stammt aus den USA. Hier übernimmt ein Arzt als medizinischer Direktor, häufig in Teilzeit, die Kontrolle und Organisation der medizinischen Versorgung im Pflegeheim. So ist er verantwortlich für andere Ärzte, die Bewohner in der Einrichtung betreuen, aber zusätzlich oft auch selbst in der medizinischen Grundversorgung der Bewohner tätig (Eskildsen und Price 2009). Demgegenüber stehen andere Versorgungsformen, die hier kurz erläutert werden, weil im Folgenden Studien aus diesen Ländern beschrieben werden. So existiert in den Niederlanden eine spezialisierte 
Form des Facharztes für Allgemeinmedizin (niederländisch: „Verpleeghuisarts“) der Heimhausarzt, welcher die hausärztliche Versorgung im Pflegeheim sicherstellt. In Frankreich hingegen übernimmt in der Regel ein Arzt mit geriatrischen Qualifikationen im Pflegeheim die Aufgabe des "Médecin Coordinateur" (MC), der die Koordination und Organisation rund um die medizinische Versorgung sicherstellen soll. Für gewöhnlich versorgt dieser dort keine eigenen Patienten; es gibt jedoch auch Ausnahmen, bei denen Hausärzte die Funktion des MC nebenberuflich ausüben (van den Bussche et al. 2009b).

Relativ häufig beforscht wurden die Themen der Zufriedenheit mit der Kommunikation und Kontakten zum Arzt im Pflegeheim sowie der medizinischen Versorgung am Lebensende.

\subsubsection{Perspektiven und Bedürfnisse der Angehörigen hinsichtlich der medizinischen Versorgung}

International ergeben sich in verschiedenen Studien unterschiedliche Ansichten und Erwartungen an die medizinische Versorgung im Pflegeheim.

In einer norwegischen Studie äußerten Angehörige von Pflegeheimbewohnern in Fokusgruppen generell ihr Vertrauen darüber, dass die medizinischen Bedürfnisse der Bewohner erfüllt würden (Vinsnes 2012). Indessen war in einer quantitativen amerikanischen Erhebung immerhin ein Fünftel der befragten Bezugspersonen zuvor im Pflegeheim Verstorbener der Meinung, dass das Personal nicht genug über die medizinische Vorgeschichte wusste, um die beste Versorgung am Lebensende aufzubieten (Teno et al. 2004).

In einer amerikanischen Studie wurde der Zusammenhang zwischen der Anwesenheit von Angehörigen im Pflegeheim und der Qualität der medizinischen Versorgung der Bewohner untersucht. Hierbei wurde festgestellt, dass Bewohner, die zwischen 1-7 h pro Woche Besuch erhielten, im Vergleich zu solchen, die keine Besuche oder mehr als 7 Stunden in der Woche besucht wurden, weniger an Dekubitus, Schmerzen und Dyspnoe litten. Zudem wurden diese Bewohner weniger häufig ins Krankenhaus eingewiesen. Die Autoren schlussfolgerten, dass möglicherweise eine schlechte Versorgung zu vermehrter Anwesenheit von Angehörigen führen würde (Grabowski und Mitchell 2009).

Inwiefern Wünsche für die medizinische Behandlung am Lebensende im Vorfeld diskutiert wurden, erhoben amerikanische Forscher bei Bezugspersonen zuvor im 
Heim Verstorbener. Gefragt wurde auch, ob die Angehörigen das Gefühl hatten, der Arzt höre innen zu, ob der Inhalt des vom Arzt Gesagten verständlich war und ob die Bezugspersonen die Möglichkeit erhielten, Fragen zu stellen und sich verstanden fühlten. Durchschnittlich ergab sich ein Wert von 1,5 auf einer Skala von schlecht (0) bis ausgezeichnet (3) für die Kommunikation, wobei keine Einzelwerte für die jeweiligen Items veröffentlicht wurden (Sloane et al. 2008).

Mangelnder Kontakt zu den Ärzten ist eine Quelle für Unzufriedenheit seitens der privaten Bezugspersonen. So gaben $31 \%$ der 487 Befragten in einer quantitativen Studie an, dass sie in der Sterbesituation des Bewohners keinen Kontakt zum Arzt hatten, obwohl der Wunsch bestand (Teno et al. 2004). Auch in der angeschlossenen qualitativen Follow-Up-Studie stellte sich als ein Hauptthema heraus, dass Bezugspersonen die Ärzte im Pflegeheim zu selten oder sogar nie antrafen und einige Befragte die Kommunikation mit den Medizinern bemängelten. So blieb in einigen Fällen das Bedürfnis nach Informationen über den Gesundheitszustand des Bewohners nur deswegen unerfüllt, weil die Ärzte sehr schwer zu erreichen waren (Shield et al. 2005). Auch in einer anderen quantitativen Studie zeigten sich Bezugspersonen, die zu verschiedenen Punkten der Versorgung im Pflegeheim befragt wurden, am unzufriedensten mit dem Kontakt zum Arzt. Innerhalb einer vierteiligen Skala (1 = überhaupt nicht zufrieden, 4 = sehr zufrieden) gaben sie die Zufriedenheit diesbezüglich durchschnittlich mit 3 an. Die Qualität der ärztlichen Versorgung wurde im Durchschnitt mit 3,2 bewertet (Levy-Storms und Miller-Martinez 2005). In einer schwedischen Studie zeigten sich hingegen über $90 \%$ der 526 befragten Angehörigen zufrieden mit den Arztkontakten der Pflegeheimbewohner und den ihnen übermittelten Informationen hinsichtlich gesundheitlicher Veränderungen (Berglund 2007). Ebenso gaben über $80 \%$ der befragten Bezugspersonen in einer amerikanischen Studie an, mit dem Arzt im Pflegeheim vertraut zu sein, wobei weniger als zwei Drittel den Mediziner überhaupt einmal getroffen hatten (Sloane et al. 2008).

Im Hinblick auf Entscheidungen über die medizinische Versorgung von Pflegeheimbewohnern wurden in verschiedenen Studien Aussagen von privaten Bezugspersonen getroffen: So schrieben Angehörige in einer Fokusgruppenbefragung in den USA dem Arzt eine Schlüsselrolle in der Kommunikation von Informationen innen gegenüber zu. Die privaten Bezugspersonen von zuvor in Pflegeheimen, Krankenhäusern, Hospizen und häuslicher Versorgung verstorbe- 
nen Personen, wären rückblickend auch gerne hinsichtlich der medizinischen Versorgung in getroffene Entscheidungen involviert worden (Teno et al. 2001).

Hinsichtlich der Entscheidungsfindung bei der medizinischen Versorgung und der Situation am Lebensende der Heimbewohner wurden zudem relativ häufig speziell Angehörige von Demenzerkrankten befragt.

In einer quantitativen, amerikanischen Studie zeigten sich Angehörige dementer Pflegeheimbewohner bereits sehr zufrieden mit den erhaltenen Informationen und Hilfestellungen bei der Entscheidungsfindung in medizinischen Fragen. So bewerteten fast $90 \%$ das Ausmaß der Hilfe durch das Personal der Primärversorgung in der Entscheidungsfindung auf einer 5-stufigen Skala positiv (exzellent, sehr gut oder gut). Fast ebenso viele (85\%) äußerten sich positiv über die erhaltenen Informationen zu medizinischen Problemen und Behandlungsmethoden. Die niedrigsten Zufriedenheitswerte (angemessen oder schlecht, $19 \%$ ) wurden bei der Frage zur Unterstützung und Bestärkung durch das Personal der Primärversorgung im Entscheidungsprozess angegeben. Insgesamt war die Zufriedenheit mit dem Ablauf der Entscheidungsfindung signifikant niedriger als mit der Entscheidung selbst (Givens et al. 2009).

Private Bezugspersonen dementer Bewohner am Lebensende, die dem Personal von vorneherein aufgrund dessen Status als Professionelle vertrauten, verspürten in einer qualitativen Befragung in den USA nicht unbedingt das Bedürfnis, sich an medizinischen Entscheidungen zu beteiligen. Anderen erschien es erst nach aufgebautem Vertrauen zum Personal möglich, bestimmte medizinischen Entscheidungen abzugeben. Dieses Vertrauen begründete sich hier unter anderem auf dem regelmäßigen Kontakt zum Personal und der Informierung der Angehörigen über den Verlauf der Krankheit und deren Behandlung (Caron et al. 2005).

Demgegenüber erschien es Angehörigen von Pflegeheimbewohnern mit fortgeschrittener Demenz in einer anderen qualitativen, amerikanischen Studie teilweise aufgrund mangelnder Kommunikation mit den Ärzten nicht möglich, die Meinung der Pflegeheimbewohner bei medizinischen Ereignissen zu vertreten oder sich an Entscheidungen zu beteiligen. Einige private Bezugspersonen äußerten deshalb im Krankheitsfall der Pflegeheimbewohner den Wunsch nach besserer Information, auch über die vorhandenen Behandlungsoptionen, um stellvertretend besser Entscheidungen für diese treffen zu können. Sie zeigten 
sich zwar mehrheitlich zufrieden mit der Kommunikation zum Pflegepersonal, empfanden den Austausch mit den Ärzten jedoch fast einhellig als unbefriedigend (Givens et al. 2012).

Laut Angehörigen von Pflegeheimbewohnern mit Demenz sollten behandelnde Ärzte auch routinemäßig den Patienten oder stellvertretend die Familie zu Wünschen im Hinblick auf das Lebensende befragen. Dies befürworteten in einer niederländischen Fragebogenerhebung $88 \%$ der 136 befragten Bezugspersonen voll $(78 \%)$ oder mehrheitlich (10\%). Die eingeschlossenen Heimbewohnern hatten jedoch in den wenigsten Fällen eine Patientenverfügung verfasst $(7 \%)$, in der derartige Wünsche verbindlich festgehalten werden (Rurup et al. 2006).

So wünschten sich Angehörige von Demenzkranken am Lebensende in einer qualitativen Befragung häufigere offizielle Treffen mit den Professionellen, ohne sich dabei auf eine spezielle Berufsgruppe (Ärzte, Pflege ect.) festzulegen. Diese Treffen könnten aus Angehörigensicht dazu beitragen, den Krankheitsprozess ihrer Familienmitglieder besser zu verstehen, Fragen stellen zu können, Zweifel auszuräumen und das Gefühl von Einbeziehung zu erhalten (Caron et al. 2005).

\subsubsection{Perspektiven der Angehörigen hinsichtlich ihrer Aufgaben}

Private Bezugspersonen übernehmen im Pflegeheim einige Aufgaben. Eine Befragung zu den jeweiligen Ansichten über Zuständigkeiten von Angehörigen und Mitgliedern des Personals in Nordirland ergab, dass Familien die Verwirklichung einer guten Beziehung zu den Ärzten sowohl als eigene Aufgabe, aber auch als Aufgabe des Pflegeheimpersonals sahen. Das Personal hingegen betrachtete dies eher ausschließlich als eigene Aufgabe. Bei der Bereitstellung rezeptfreier Medikamente sahen die Familien sich auch in der Verantwortung, wohingegen das Personal dies eher als seinen alleinigen Zuständigkeitsbereich betrachtete (Ryan und Scullion 2000).

In einer ähnlichen Erhebung auf Grundlage desselben Fragebogens (Shuttlesworth et al. 1982) lag nach Meinung beider Gruppen die Bereitstellung nicht-rezeptpflichtiger Medikamente hingegen eher im Zuständigkeitsbereich der Familien. Sowohl Angehörige als auch Pflegeheimpersonal sahen allerdings die Entscheidung über eine Krankenhauseinweisung des Bewohners als alleinige Personalaufgabe an (Schwartz und Vogel 1990). 
In einer amerikanischen Erhebung von Port et al. 2005 gaben rund zwei Drittel der befragten Angehörigen von Pflegeheimbewohnern und betreut wohnenden Bewohnern an, bereits einmal in deren medizinische Versorgung involviert gewesen zu sein. Dies implizierte, dass Angehörige mit dem Personal oder dem zuständigen Arzt über die Medikation, die Ernährung oder gesundheitliche Probleme des Bewohners gesprochen hatten. Die Autoren fanden zudem heraus, dass Angehörige von betreut wohnenden Bewohnern, die entsprechend keine 24Stunden-Betreuung bekamen, häufiger in die medizinische Versorgung der Bewohner involviert waren, als Angehörige von Pflegeheimbewohnern.

In mehreren Studien wird deutlich, dass es Angehörige von Pflegeheimbewohnern mitunter als ihre Aufgabe verstehen, sich einer guten, vor allem pflegerischen Versorgung der Bewohner zu versichern (Keefe und Fancey 2000, Sandberg et al. 2001). Aus einer qualitativen, amerikanischen Erhebung geht zudem hervor, dass Angehörige immer dann zu „Anwälten“ (Wetle et al. 2005 S. 646) ihrer umsorgten Pflegeheimbewohner wurden, wenn die pflegerische, aber auch die medizinische Versorgung nicht ihren Vorstellungen entsprach. Dies beinhaltete etwa, dass sich Angehörige bei Änderungen des Gesundheitszustandes des Bewohners für eine Versorgung einsetzten (Wetle et al. 2005).

\subsubsection{Stellvertreterfähigkeit der Bezugspersonen für Bewohnerperspektiven}

In vielen Fällen können Bewohner im Pflegeheim aufgrund kognitiver Einschränkung beispielsweise durch Demenz, ihre Interessen nur noch eingeschränkt oder gar nicht mehr vertreten. Um herauszufinden, inwieweit private Bezugspersonen von Pflegeheimbewohnern deren Meinung generell widerspiegeln können, wurden beide Akteure in Studien zur Qualität der Versorgung im Pflegeheim befragt und die Antworten verglichen.

So untersuchten französische Forscher die Meinungen von 125 BewohnerBesucher-Paaren. Themen der Befragung waren dabei unter anderem die Zufriedenheit mit der medizinischen/ pflegerischen Versorgung und die bereitgestellten Informationen. Es sollte hinsichtlich der medizinischen Versorgung etwa angegeben werden, ob alles getan wurde, um den Bewohner von Schmerzen zu befreien und wie die Zufriedenheit mit der Erreichbarkeit von Personal zu verschiedenen Zeiten sei. Im Hinblick auf bereitgestellte Informationen wurde auch die Zufriedenheit mit Erklärungen zur Krankheit des 
Bewohners, dessen Behandlung und dem Zweck von Untersuchungen erfragt. Die Bezugspersonen sollten dabei so antworten, wie sie dachten, dass es der Bewohner tun würde. Um die Antworten der beiden Akteursgruppen besser vergleichen zu können wurde ein Intraklassen-Korrelationskoeffizient (ICC) errechnet, der Werte im Bereich von 0 bis 1 einnehmen konnte. Der Grenzwert für eine nicht zufriedenstellende Übereinstimmung der jeweiligen Antworten lag bei $\leq$ 0,4 und wurde sowohl hinsichtlich der medizinisch/pflegerischen Versorgung (ICC $=0,08)$ als auch bezüglich der bereitgestellten Informationen $(I C C=0,18)$ deutlich unterschritten. Hieraus schlossen die Autoren, dass die Bezugspersonen nicht in der Lage waren, verlässliche Informationen zur Bewohnerzufriedenheit anzugeben, da sie diese systematisch überschätzten (Gasquet et al. 2003).

Bereits 1992 hatten amerikanische Forscher Bewohner mit durchschnittlich milder bis mäßiger kognitiver Einschränkung (gemäß Mini-Mental-Status-Test) und deren Bezugspersonen mit derselben Intention wie Gasquet et al. befragt. Es wurde jedoch ein anderer Fragebogen angewandt, der unter anderem die von den Angehörigen vermutete Bewohnerzufriedenheit mit ärztlichen Diensten (Professionalität, medizinische Behandlung, mit dem Bewohner verbrachte Zeit, Erreichbarkeit, Beziehung des Befragten zum Arzt, Bereitschaft/ zufriedenstellende Fähigkeit des Arztes Fragen/ Anliegen zu beantworten, Vertrauen in ärztliche Qualifikation) untersuchte. Auf einer vierstufigen Skala, bei der 4 die höchste Zufriedenheit (sehr gut) darstellte, bewerteten die privaten Bezugspersonen die einzelnen Items nie schlechter als gut (3). Dies galt auch für andere Subskalen, bei der unter anderem die Zufriedenheit mit der medizinischen Behandlung durch das Pflegepersonal erfragt wurde. Die Forscher stellten eine durchgängig positivere Bewertung durch die Angehörigen, verglichen mit den Bewohnern, fest (Lavizzo-Mourey et al. 1992).

\subsection{Zusammenfassung}

Aus den vorliegenden deutschen Studien lassen sich lediglich punktuelle Erkenntnisse über selbst attribuierte Aufgaben der Angehörigen im Pflegeheim und deren Ansichten zur medizinischen Versorgung gewinnen, die Studien setzen jedoch nicht ihren Schwerpunkt darauf. Es ließ sich erkennen, dass Angehörige die ärztliche Versorgung und auch den Informationswunsch hinsichtlich der medizinischen Versorgung in verschiedenen Studien sehr unterschiedlich 
bewerteten. So wurden die Qualität der Versorgung und die Erreichbarkeit der Ärzte in einer Erhebung als gut eingestuft, in einer anderen Studie hingegen äußerte sich nicht einmal ein Drittel zufrieden mit der ärztlichen Versorgung. In den internationalen Erhebungen wurden zum Teil globale Zufriedenheitswerte ermittelt, ein besonderes Augenmerk lag insgesamt auf Kommunikation und Kontakten zum Arzt. So war mangelnder Kontakt zum Arzt eine Quelle für Unzufriedenheit. Darüber, inwieweit die Bezugspersonen in medizinische Entscheidungen insbesondere am Lebensende der Bewohner einbezogen werden wollten, ergaben sich unterschiedliche Ergebnisse. Dies reichte vom generellen Wunsch nach Einbeziehung bis zum Vertrauen in die Professionellen und einer damit verbundenen Verantwortungsabgabe in Bezug auf medizinische Entscheidungen. Teilweise war dies auch mit dem Wunsch nach besserer Kommunikation mit den Ärzten verknüpft. Im Hinblick darauf, ob Bezugspersonen stellvertretend die Meinung der Pflegeheimbewohner wiedergeben können, ergab sich, dass diese die medizinische Versorgung häufig positiver bewerteten als die Bewohner selbst.

Die Ergebnisse der internationalen Erhebungen lassen nicht auf die Situation in Deutschland schließen und hierzulande fehlen umfassende Untersuchungen zu Sichtweisen und Bedürfnissen der Angehörigen von Pflegeheimbewohnern bezüglich der medizinischen Versorgung. Es erschien für diese Dissertation deshalb sinnvoll, durch eine offene Herangehensweise einen möglichst hohen Erkenntnisgewinn über die Erfahrungen und Wünsche von Angehörigen im Hinblick auf die medizinische Versorgung in Pflegeheimen zu erzielen.

\subsection{Fragestellung und Ziel}

Ziel dieser Dissertation ist es, Perspektiven und Bedürfnisse aus Angehörigensicht hinsichtlich der medizinischen Versorgung von Bewohnern in Pflegeheimen zu ermitteln. Durch die Interviews sollen Erfahrungen, Vorstellungen und Wünsche der privaten Bezugspersonen herausgearbeitet werden, die dann im Ergebnis andere Studieninhalte (siehe Kap. 4.1) ergänzen, die die Sichtweisen der anderen Akteure im Pflegeheim beleuchten. Mit dem erhaltenen Gesamtbild soll ein Beitrag zur Verbesserung der medizinischen Versorgung geleistet werden. 
Der Fokus der Befragung lag hierbei auf der Wahrnehmung der medizinischen Versorgung und der Interaktion zwischen Hausarzt und Pflege. Um Auskunft über Verbesserungsmöglichkeiten zu erhalten, erscheint es zudem sinnvoll zu erfragen, was sich Angehörige hinsichtlich der medizinischen Versorgung wünschen. Untersucht wurden daher folgende Fragestellungen:

1. Welche Erfahrungen haben die Angehörigen bisher im Pflegeheim mit der medizinischen Versorgung, Ärzten und Pflegenden gemacht? Wie wird der Hausarztbesuch erlebt? Welche Vorstellungen/ Wünsche haben die Bezugspersonen hinsichtlich der medizinischen Versorgung im Pflegeheim? Welche Ansichten haben Angehörige zu einer Kontinuität in der hausärztlichen Versorgung?

2. Wie erleben die Angehörigen die Interaktion zwischen Ärzten und Pflegekräften? Welche Erfahrungen haben sie mit der Zusammenarbeit der beiden Berufsgruppen gemacht?

3. Welche Aufgaben übernehmen Angehörige bei der medizinischen Versorgung? 


\section{Material und Methoden}

\subsection{Methodische Anlage der Untersuchung und Einbettung in die Studie „INTERPROF“}

Die Dissertation beschäftigt sich mit den Perspektiven, Bedürfnissen und Ansprüchen von Bezugspersonen von Pflegeheimbewohnern an die medizinische Versorgung, mit einem Fokus auf der Zusammenarbeit zwischen Pflegekräften und Hausärzten, die in offenen Leitfadeninterviews ermittelt wurden. Die Gespräche wurden qualitativ mittels der Methode der Grounded Theory (Strauss und Corbin 1996) ausgewertet.

Eingebettet ist die Arbeit in die qualitative, multizentrische Studie „INTERPROF Interprofessionelle Zusammenarbeit und Kommunikation im Pflegeheim“. Diese wird vom Institut für Allgemeinmedizin der Universität Göttingen, dem Institut für Allgemeinmedizin der Universität Hamburg-Eppendorf, dem Zentralinstitut für seelische Gesundheit in Mannheim und der Fachhochschule Hannover seit dem Frühjahr 2012 über 3 Jahre durchgeführt. Die Studie wird vom Bundesministerium für Bildung und Forschung gefördert (01GY1124) und untersucht die Zusammenarbeit und Kommunikation zwischen Hausärzten und Pflegekräften in Pflegeheimen. Hierfür werden Interviews mit Hausärzten, Pflegekräften, Bewohnern und Angehörigen in allen Studienstandorten geführt, um die derzeitige Situation bezüglich Kommunikation und Zusammenarbeit zwischen den Berufsgruppen zu erfassen. Außerdem sollen Perspektiven und Bedürfnisse von Heimbewohnern und Angehörigen an die medizinische Versorgung identifiziert werden. Weiterhin finden im Verlauf Fokusgruppen mit Hausärzten und Pflegenden sowie ein Expertenworkshop ${ }^{2}$ statt, um ein Kommunikationsmodell zu entwickeln und dieses nachfolgend in einer Pilotstudie zu testen. Die Doktorandin war an der Durchführung und Auswertung der Angehörigeninterviews beteiligt.

\footnotetext{
${ }^{2}$ Der Expertenworkshop setzt sich zusammen aus Hausärzten, Pflegekräften, Angehörigenvertretung, Pflegeheimdirektionen, Experten für Pflegewissenschaften, Kommunikation, Change Management und Wissenschaftlern des Projekts.
} 


\subsubsection{Begründung des qualitativen Vorgehens}

Das Forschungsinteresse liegt in den subjektiven Betrachtungsweisen und Bedürfnissen der Angehörigen im Pflegeheim bezüglich der medizinischen Versorgung. Aufgrund der offeneren Zugangsweise mit durch Art und Inhalt weniger festgeschriebenen Fragen, als es Fragebogenstudien oder standardisierte Interviews zulassen, ist eine wesentlich konkretere Darstellung der individuellen Perspektive der Teilnehmer möglich (Flick et al. 2012). Da es qualitative Methoden erlauben, wenig bekannte Phänomene nachzuvollziehen und zu erklären (Strauss und Corbin 1996), aber auch "das Unbekannte im scheinbar Bekannten" (Flick et al. 2012, S. 17) offenbaren können, wurde die Methode gewählt, um eine aktuelle Bestandsaufnahme zu ermitteln.

Qualitative Forschung bietet die Gelegenheit, Bedeutungen zu ermitteln und Aussageabsichten des Textes in einem Verstehensprozess auszulegen, da zugeschriebene Bedeutungen individuell verschieden sind. Hierin liegt der ausschlaggebende Unterschied zu standardisierten Befragungen, in denen „, $\mathrm{Ja}=$ Ja und Nein $=$ Nein ist - unabhängig von dem tatsächlichen Bedeutungsgehalt dieser Reaktionen“" (Hopf 1978 S. 100).

Den theoretischen Hintergrund bildet die Grounded Theory (siehe Kap. 4.5), die vor allem dort geeignet ist, wo eine vielschichtige soziale Wirklichkeit sich nicht allein quantitativ erfassen lässt, sondern es um, auf Sprachebene vermittelte, Interaktions- und Sinnzusammenhänge geht (Legewie 1996). Für die vorliegende Studie wurde diese Methode auch deshalb gewählt, weil sie sich besser für offene Fragestellungen eignet als beispielsweise die Qualitative Inhaltsanalyse, die sich ausdrücklich schon an bestehenden Theorien orientiert. Das offene Vorgehen bei Verwendung der Grounded Theory konzentriert sich vor allem auf die Aspekte, die in Verbindung mit den Interviewpartnern stehen und somit Rückschlüsse auf die subjektiven Bedeutsamkeitszuschreibungen zulassen. Die Resultate dieser Erhebungsmethode sind facettenreich und beinhalten unterschiedliche Abstraktionsebenen (Wollny und Marx 2009). Die Grounded Theory entspricht dem Anspruch der Studie, theoretisch untermauert auch von praktischer Relevanz für die medizinische Versorgung im Pflegeheim sein zu können. 


\subsection{Stichprobe, Auswahlverfahren und Rekrutierung der Teilnehmer}

Die Durchführung der Studie an drei aufgrund ihrer Größe und Lage unterschiedlichen Standorten (Hamburg mit 1,8 Mio. Einwohnern, Göttingen mit 120.000 Einwohnern und Mannheim mit 315.000 Einwohnern) machte eine Rekrutierung von Teilnehmern in den Städten selbst und ihren umgebenden ländlichen Regionen möglich. Es wurden Heime verschiedener Träger in städtischen sowie ländlichen Gegenden angeschrieben, die Mehrheit der Heime wurde jedoch aus städtischem Gebiet rekrutiert. Von den Standorten Göttingen, Hamburg und Mannheim wurden verschiedene Heime in schriftlicher Form oder per Telefon kontaktiert und über die geplante Studie in Kenntnis gesetzt. Das weitere Vorgehen wurde bei Interesse in einem Telefonat besprochen und ein Gesprächstermin mit der Pflegedienstleitung oder den jeweiligen Wohnbereichsleitungen festgelegt. Über die Pflegedienstleitungen/ Bereichsleitungen/ Pflegekräfte wurden Angehörige bzw. Bezugspersonen durch gezielte Ansprache oder über bereits für die Studie ausgewählte Pflegeheimbewohner rekrutiert. Zusätzlich konnten bei Einverständnis der Heime Teilnehmer über bereits an der Studie teilnehmende Hausärzte gewonnen werden. Zudem wurden Aushänge zum Projekt in den jeweiligen Wohnbereichen ausgestellt. Interessierte Angehörige setzten sich entweder über die Pflegekräfte oder direkt mit dem Projektteam in Verbindung. Die Interviewtermine wurden dann telefonisch vereinbart, wobei sich die Terminfindung problemlos gestaltete.

Für die privaten Bezugspersonen galten folgende Einschlusskriterien:

1. mindestens ein Kontakt pro Monat zum Pflegeheimbewohner

2. Hauptbezugsperson

3. unterschriebene Einverständniserklärung

4. Volljährigkeit

5. ausreichende Deutschkenntnisse 
Eine verwandtschaftliche Beziehung zum Bewohner war für die Studienteilnahme nicht erforderlich. $\mathrm{Ob}$ die potentiellen Teilnehmer tatsächlich die Einschlusskriterien erfüllten, wurde im Vorfeld durch direktes Nachfragen durch den Interviewer sichergestellt. So konnten potentiell interessierte Angehörige nicht immer in die Studie eingeschlossen werden, etwa weil sie das Kriterium der Hauptbezugsperson nicht erfüllten.

Ziel bei der Auswahl der Studienteilnehmer war die Rekrutierung gemäß dem Konzept des "Theoretical Samplings" von Strauss und Corbin (Strauss und Corbin 1996). Initial wurden die Angehörigen in der Reihenfolge ihrer Zusagen interviewt, da hier noch nicht klar war, welche Konzepte theoretisch relevant werden. So wurden potenziell bedeutsame Konzepte ermittelt und deren Merkmale und Ausmaße ausfindig gemacht. Im Verlauf des Analyseprozesses sollten gezielt Teilnehmer für einen weiteren Erkenntnisgewinn eingeschlossen werden. Die Auswahl der Teilnehmer sollte dann nach ihrer Relevanz für die bereits bestehenden Konzepte erfolgen, um diese zu überprüfen, aber auch um Kontrastierung durch Gegenbeispiele herauszufiltern (Strauss und Corbin 1996). Die Umsetzung des "Theoretical Samplings" konnte jedoch, da sich die Rekrutierung potentieller Teilnehmer als schwer erwies, nur ansatzweise durchgeführt werden. Die Rekrutierung erfolgte deshalb auch im Verlauf hauptsächlich anhand der Einschlusskriterien und weiterhin eher nach der Reihenfolge der Zusage. So war ein Problem bei der Durchführung der Rekrutierung, dass häufig bereits die Pflegedienstleitungen einer Teilnahme an der Studie nicht zusagten und deshalb Angehörige aus diesen Heimen nicht angesprochen werden konnten. Nur in den einbezogenen Heimen wurden wiederholt Pflegekräfte und Heimleitungen angesprochen und um die Ansprache weiterer Bezugspersonen gebeten. Dieser Prozess gestaltete sich als langwieriger und schwieriger als im Vorfeld angenommen.

\subsection{Die Erhebungsmethode}

\subsubsection{Das offene Leitfadeninterview}

Das offene Leitfadeninterview ist in der qualitativen Forschung dazu geeignet, Forschungsthemen mit relativ klar umrissenen Fragestellungen nachzugehen (Przyborski und Wohlrab-Sahr 2010). Es zählt zu den teilstandardisierten 
Interviews. Die Forscher orientieren sich hierbei an einem Interviewleitfaden, haben jedoch gleichzeitig viele Freiräume für Formulierungen, Nachfragemethoden und die Reihenfolge der Fragen (Hopf 2012).

Ziel dieser Form der Erhebung ist es, die Fragen so zu stellen, dass bestimmte Sachverhalte und Problematiken in ihrem jeweiligen Kontext und Bedeutungszusammenhang erfasst und nachvollzogen werden können. So ist es möglich, Informationen über Bedingungen für das Entstehen und die Relevanz bestimmter Phänomene zu gewinnen. Die thematischen Interessen des Forschers müssen sich im Interview deshalb an den von den Befragten gesetzten inhaltlichen Schwerpunkten orientieren, anstatt an den vorab skizzierten Ordnungen und Strukturierungen des Untersuchers (Przyborski und Wohlrab-Sahr 2010). Nur so ist es möglich, auch Hinweise auf ergänzende Aspekte, die für die medizinische Versorgung im Pflegeheim von Bedeutung sind, zu ermitteln und nicht in eine so genannte "Leitfadenbürokratie“ (Hopf 1978 S. 101) zu verfallen, bei der sich der Interviewer starr am Leitfaden orientiert und damit eine Vergleichbarkeit im Sinne von quantitativen Methoden vortäuscht. Werden Problemzusammenhänge geschildert, die im Leitfaden ursprünglich gar nicht eingeschlossen waren, ist es angeraten, darauf einzugehen und gegebenenfalls die Themenbreite zu erweitern (Przyborski und Wohlrab-Sahr 2010).

Das Interview soll so „ungekünstelt“ wie möglich sein, ohne sich die Regeln der Kommunikation im Alltag anzueignen. Die Rollentrennung zwischen Frager und Befragtem bleibt bestehen. Die Schwierigkeit für den Interviewer besteht darin, seinen spontanen Neigungen, den Regeln der Altagskommunikation zu folgen, zurückzuhalten. Das bedeutet, dass er keine Deutungen anbietet, mitredet, von sich spricht oder Inhalte zusammenfasst (Hopf 1978).

\subsubsection{Leitfaden und Demografiebogen}

„Der Leitfaden dient dem Interview und nicht das Interview dem Leitfaden.“

(Przyborski und Wohlrab-Sahr 2010, S.142)

Der Leitfaden wurde in Zusammenarbeit von Studienmitarbeitern und der Soziologin Dr. Gabriella Marx erstellt. Dabei wurde zunächst ermittelt, welche Erkenntnisse mit den Interviews gewonnen werden sollten. Anhand der aktuellen Studienlage wurden die als wichtig erachteten Themenkomplexe identifiziert. Die 
Fragen wurden in einem Diskussionsprozess immer wieder bearbeitet und präzisiert, wobei man besonders auf die Verständlichkeit und die Relevanz der jeweiligen Frage achtete.

Um dem Prinzip des offenen Leitfadeninterviews gerecht zu werden, soll zu Beginn eine offene, weitgesteckte, erzählgenerierende Frage gestellt werden. Daher wurde als Intervieweinstieg die Frage nach dem typischen Ablauf eines Besuches der Bezugsperson im Pflegeheim gewählt. Dem Interviewpartner sollte damit die Möglichkeit gegeben werden, den Sachverhalt aus seiner Sicht zu umreißen und gegebenenfalls hier schon eine Problemsicht aufzuzeigen, die sich im Verlauf durch zunehmende Spezifität entwickeln lässt (Merton et al. 1990; Hopf 1978). An diese Ausführungen sollten dann spezifischere Nachfragen etwa zu Erfahrungen mit der Pflege und der ärztlichen Versorgung angeschlossen werden. Weitere Themenbereiche, die für die Studie interessant waren, sollten nach demselben Prinzip erschlossen werden. So wurde zunächst generell nach einer Erinnerung zum letzten Hausarztbesuch beim Heimbewohner und den Eindrücken über Zuständigkeitsbereiche der Pflege und der Ärzte im Pflegeheim gefragt, an die sich dann in die Tiefe gehende Fragen anschlossen.

Zum Abschluss des Interviews wurden Vorstellungen zur idealen Versorgung im Pflegeheim erfragt, um eine Evaluation oder Bewertung anzustoßen.

Das Forschungsinteresse lag hierbei sowohl in generellen Perspektiven zur medizinischen Versorgung im Pflegeheim, als auch in spezifischen Beschreibungen von Vorgängen und Regelungen.

Im Verlauf der Erhebung wurde der Leitfaden auf Grundlage des bisher erhobenen Materials dahingehend angepasst, dass den Bezugspersonen die Frage, wie ihnen der Alltag im Heim gefällt oder nicht gefällt, nicht mehr gestellt wurde.

Übersicht über die Themenfelder des Interviewleitfadens für Angehörige:

1. Erzählung eines typischen Besuchs im Pflegeheim

- Erfahrungen im Pflegeheim hinsichtlich der Pflege

- Kontinuität der medizinischen Versorgung

- ärztliche Versorgung 
- Beschreibung eines typischen Hausarztbesuches

2. Beschreibung des letzten Hausarztbesuches

- Gründe, Ablauf, Erleben

3. Erfahrungen mit Zuständigkeitsbereichen und Aufgabenverteilungen

- Jeweilige Zuständigkeitsbereiche der Ärzte und des Pflegepersonals

- Erleben der Zusammenarbeit

- Aufgaben des Angehörigen

- Tätigkeiten, die der Bewohner noch selbst übernimmt

4. Vorstellungen über die ideale Situation/ medizinische Versorgung im Pflegeheim

- Vorstellungen der Angehörigen zum idealen Pflegeheim, der idealen Versorgung

Zusätzlich wurden in einem Kurzfragebogen demografische Daten erfasst wie:

- Alter

- Geschlecht

- Verhältnis zum Bewohner

- Existenz einer gesetzlichen Betreuung und wenn ja ob der Befragte der Betreuer ist

- Dauer des Aufenthalts des Bewohners in der Einrichtung

- Durchschnittliche gemeinsam verbrachte Zeit mit dem Bewohner

- höchster Schulabschluss

- berufliche Tätigkeit

Diese Angaben sollten erhoben werden, jedoch ohne den Gesprächsverlauf zu unterbrechen. Im Verlauf der Interviewdurchführungen wurde dieser Demografiebogen angepasst und noch Informationen dokumentiert zur: 
- Ausrichtung des Einrichtungsträgers (kommunal, frei-gemeinnützig, privat)

- Anzahl der Plätze in der Einrichtung

- Umgebung der Einrichtung (<5000 Einwohner, > 5000 Einwohner)

Des Weiteren wurden die Frage zur gemeinsam verbrachten Zeit konkretisiert und nun nach der Häufigkeit der Besuche pro Monat und der gemeinsam verbrachten Zeit pro Monat gefragt.

\subsection{Datenerhebung}

\subsubsection{Interviewdurchführung}

Von den Göttinger Interviews wurde ein Teil $(n=2)$ von der Doktorandin selber durchgeführt. Dies ergab sich aus der Tatsache, dass die Befragungen an drei verschiedenen Standorten innerhalb Deutschlands stattfanden und die Doktorandin im Verlauf des Projekts dazukam, als bereits einige Interviews geführt worden waren.

Die Interviews wurden, je nach Angehörigenwunsch, unter verschiedenen Rahmenbedingungen, im Pflegeheim selbst, in der häuslichen Umgebung der Angehörigen oder auch in den jeweiligen Instituten geführt. Es wurde versucht eine ruhige Atmosphäre möglichst ohne Unterbrechungen oder äußere Störungen zu schaffen, was sich jedoch nicht immer vollständig umzusetzen ließ. Einige Gespräche fanden auch unter Beisein der Pflegeheimbewohner oder mit zwei Angehörigen gleichzeitig, jeweils auf ausdrücklichen Wunsch hin, statt.

Zu Beginn des Zusammentreffens wurden noch einmal das Anliegen der Studie und der Verlauf des Gesprächs erläutert. Die Interviewpartner wurden gebeten, den Kurzfragebogen auszufüllen und unterschrieben die Einverständniserklärung zur Studienteilnahme.

Im Anschluss wurde das Aufnahmegerät angeschaltet und das Interview mit der oben beschriebenen Einstiegsfrage eingeleitet.

Die Interviewfragen an die Angehörigen wurden so gestellt, dass sie möglichst offen beantwortet werden konnten und zum selbstständigen Erzählen anregten. Die Fragen sollten sich am Leitfaden orientieren, durften in ihrer Reihenfolge 
jedoch flexibel an den Gesprächsverlauf angepasst werden. Zunächst sollte nicht vom Interviewer interveniert werden. Er übernahm hier die Rolle des aktiven Zuhörers und griff erst im anschließenden Nachfrageteil offen gebliebene Fragen auf (Hopf 2012). Viele der interviewten Angehörigen antworteten von sich aus bereits erzählfreudig auf die offen gestellten Fragen, andere konnten erst durch Nachfrage zu ausführlicheren Schilderungen angeregt werden. In den Nachfragen gelang es den Interviewern nicht immer den offenen Fragestil beizubehalten, was bei der Auswertung entsprechend berücksichtigt wurde. Des Weiteren zeigte sich in den Interviews, dass viele Angehörige ihre Erzählschwerpunkte eher bezüglich des Heimalltag und der allgemeinen Versorgung der Bewohner als speziell der medizinischen Versorgung setzten. Auch wurden mitunter bei Fragen zur medizinischen Versorgung eher von Aspekten der pflegerischen Versorgung berichtet.

Alle Gespräche wurden mit Diktiergeräten aufgezeichnet und anschließend transkribiert und pseudonymisiert. Zusätzlich wurde jeweils ein Kontextprotokoll im Anschluss an das Gespräch erstellt, welches durch das gesprochene Wort nicht deutlich werdende Beobachtungen und Eindrücke während des Interviews festhält.

Die Interviews konnten im Verlauf der Studie problemlos durchgeführt werden. Wir schlossen die Rekrutierung mit dem Erreichen einer hinreichenden Datendichte entsprechend des Konzepts der theoretischen Sättigung ab (Strauss und Corbin 1996).

\subsection{Datenauswertung}

\subsubsection{Die Grounded Theory Methode}

Flick übersetzte 2007 Grounded Theory mit "Gegenstandsbegründete Theorie" und findet hiermit eine passende Beschreibung für die Methode, bei der schrittweise induktiv aus empirisch erhobenen Daten begründete Theorien über bestimmte Phänomene entwickelt werden. Die von Glaser und Strauss 1967 erstmals in "The Discovery of Grounded Theory" veröffentlichte Methode, beinhaltet Empfehlungen für Auswertungstechniken „um über die soziale Wirklichkeit nachzudenken und sie zu erforschen" (Strauss und Corbin 1996 S. X). 
Der Begriff Grounded Theory wird hierbei sowohl für das systematische Auswertungsverfahren als auch für das Resultat dieses Prozesses genutzt.

Ziel der Methode ist es nicht bereits bestehenden Theorien nachzugehen, sondern, erst im Verlauf der Erhebung festzustellen, was im Untersuchungsbereich von Bedeutung ist. Die Sammlung von Daten, deren Analyse und die dazu entwickelte Theorie stehen in einem zirkulären Prozess. Das bedeutet aufgestellte Theorien müssen immer wieder an den Daten überprüft werden.

Den Autoren zufolge muss eine Grounded Theory vier zentrale Kriterien erfüllen: „Übereinstimmung, Verständlichkeit, Allgemeingültigkeit und Kontrolle“ (Strauss und Corbin 1996 S.8). Eine Theorie sollte demnach mit der Wirklichkeit des untersuchten Gegenstandsbereiches übereinstimmen und nachvollziehbar sein. Sie bedarf hinreichender Abstraktheit, um auch in anderen Kontexten, die eine Verbindung zum erforschten Phänomen aufweisen, verwendet werden zu können. Zusätzlich sind die Voraussetzungen, auf die sich die Theorie bezieht, klar aufzuzeigen (Strauss und Corbin 1996).

\subsubsection{Anwendung der Grounded Theory Methode}

Die Auswertung der Interviews erfolgte in einem Forscherteam, das nach den Empfehlungen von Strauss und Corbin verfuhr (Strauss und Corbin 1996). Zunächst wurden die Interviews pseudonymisiert und anschließend in voller Länge verbatim transkribiert. Die Doktorandin und ein weiterer, in der Grounded Theory Methodik geschulter, Projektmitarbeiter werteten die Interviews gemeinsam aus. Dabei setzte sich zunächst einer der beiden mit dem entsprechenden Interview auseinander, welches anschließend vom jeweils anderen gegengelesen und dann gemeinsam bearbeitet wurde. Hierbei wurde das für qualitative Forschungsarbeiten entwickelte Computerprogramm MAXQDA benutzt.

Ein besonderes Augenmerk bei der Analyse mittels Grounded Theory (Strauss und Corbin 1996) lag hierbei auf Bedürfnissen und Problematiken hinsichtlich der medizinischen Versorgung sowie der interprofessionellen Zusammenarbeit. Es sollten verschiedene Verhaltensweisen, Perspektiven, Erfahrungen und Empfindungen analysiert und auch Wünsche beziehungsweise Ansprüche der Befragten identifiziert werden. Es wurden keine hypothetischen Vorannahmen 
untersucht, sondern im Verlauf entwickelte Theorien, anhand weiterer Interviews immer wieder überprüft und inhaltlich komplettiert. Die Forscher waren dazu angehalten, fortwährend mit ihren Daten in Interaktion zu treten (Hildenbrand 2012).

Es wurden drei Arten des Codierens eingesetzt: offenes, axiales und selektives Codieren.

Den ersten Schritt des Analyseprozesses stellte das offene Codieren dar. Hierbei wurden im inhaltlichen Zusammenhang stehende Textabschnitte identifiziert und mit einer Überschrift (Code) versehen, die sich unmittelbar auf die Daten bezieht. In dem Zusammenhang wurden die W-Fragen (Wer; Was; Wie; Warum; Wozu etc.) an den Text gestellt, um über eine schlichte Umschreibung der Daten hinauszukommen (Böhm 2012). Die Konzepte waren stets vorläufig und wurden im Verlauf angepasst. Später ließen sich verschiedene Konzepte durch das Anstellen von Vergleichen zu Kategorien zusammenfassen, wenn sie sich auf ein ähnliches Phänomen bezogen. Die Namen der Kategorien beschreiben ihren Inhalt dabei auf einer abstrakteren Ebene und konnten in Unterkategorien, die sich auf verschiedene Eigenschaften und ihre jeweiligen Dimensionen beziehen, aufgesplittet werden (Strauss und Corbin 1996).

Zusätzlich zu den Codes wurden während des gesamten Prozesses "CodeNotizen"(Strauss und Corbin 1996 S.54) als eine Form von Memo erstellt, in denen weiterführende Wahrnehmungen, Interpretationen und Fragen festgehalten wurden.

Im weiteren Verlauf wurde das axiale Codieren dazu eingesetzt, Beziehungen zwischen den Kategorien und ihren jeweiligen Unterkategorien durch repetitive Fragen und Vergleiche an das Datenmaterial zu erarbeiten. Damit diente es der "Verfeinerung und Differenzierung“ (Böhm 2012, S. 478) der Kategorien. Zur Entwicklung dieser Relationen wurde das Codierparadigma nach Strauss und Corbin angewendet. Dieses Modell ermöglicht die Darstellung von ursächlichen Bedingungen, Kontext und intervenierenden Bedingungen, Handlungsstrategien und Konsequenzen, die sich hinsichtlich der zentralen Phänomene ergeben (Strauss und Corbin 1996).

Zu den Kontextbedingungen gehören hierbei vor allem Ort, Zeit und Dauer. Intervenierende Bedingungen beziehen sich hingegen auf die individuelle 
Lebensgeschichte und die soziale, politische und kulturelle Umwelt der befragten Personen (Böhm 2012).

Die hypothetischen Beziehungen mussten sich immer wieder an den Daten bewähren. Dabei wurde auch auf Beispiele geachtet, die diese Hypothesen nicht bestätigten. Dieser Prozess war durch einen ständigen Wechsel zwischen „aufstellen“ und „verifizieren“ von Aussagen über die Beziehungen, Eigenschaften und Dimensionen der Kategorien gekennzeichnet und diente der Verdichtung und Variation der Kategorien.

Nach Strauss und Corbin (Strauss und Corbin 1996, S. 89) ist dieser Vorgang das, was eine Grounded Theory „gegenstandsverankert“ bzw. „bodenständig“ macht.

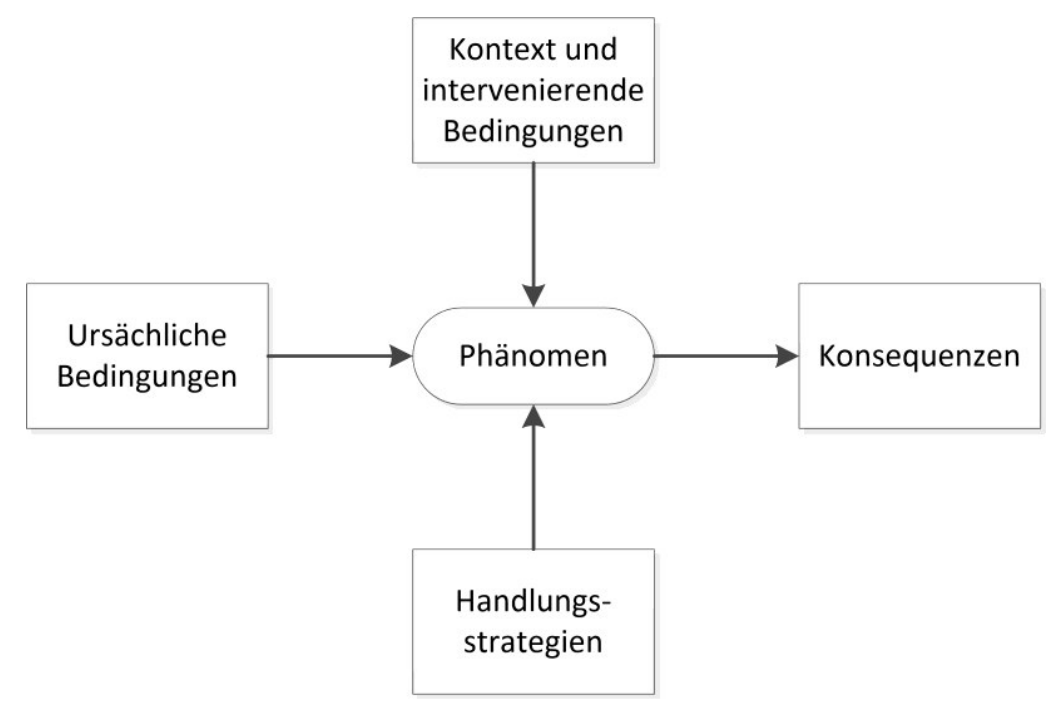

Abb. 1 Codierparadigma nach Strauss und Corbin (Böhm 2012, S.479), die Verwendung der Abbildung erfolgt mit freundlicher Genehmigung des Rowohlt-Verlages

Das selektive Codieren zielte darauf ab, eine Kernkategorie und deren Eigenschaften herauszufiltern, die zu allen anderen wichtigen Kategorien in diversen Beziehungen steht. Diese Verbindungen wurden erneut mithilfe des Codierparadigmas ermittelt. Das daraus gewonnene theoretische Konzept umfasst Bedingungen, Kontext, Strategien und Konsequenzen des Forschungsbereiches. Hypothesen über die kategorialen Beziehungen und die daraus abgeleitete Theorie wurden immer wieder am Datenmaterial validiert. 
Mit dem Erreichen einer hinreichenden Datendichte schlossen wir gemäß dem Konzept der theoretischen Sättigung (Strauss und Corbin 1996) die Rekrutierung der Studienteilnehmer ab. Es kann demgemäß davon ausgegangen werden, dass mit zusätzlichen Befragungen keine neuen Erkenntnisse mehr gewonnen werden.

Im Ergebnisteil dieser Dissertation spiegelt sich der Auswertungsprozess wider. So sind zunächst noch die Ergebnisse des offenen Codierens beschrieben, um im Anschluss vertiefend auf die Ergebnisse des axialen und selektiven Codierens einzugehen. Die Doktorandin hat sich hierbei auf die Vertiefung der inhaltlich besonders interessanten Teile, dem Einbezogensein der Bezugspersonen in die medizinische Versorgung, konzentriert. Dies ergab sich auch aus der Einbindung ins Projekt "INTERPROF“, so wurden etwa Themen wie die Zusammenarbeit zwischen Ärzten und Pflegekräften (Kap. 5.3.3) innerhalb des Projekts umfassender bearbeitet und entsprechend in der vorliegenden Dissertation nur auf der Ebene des offenen Codierens dargelegt.

Die Qualitätssicherung der Dissertation hinsichtlich Kriterien der guten qualitativen Forschung wurde anhand folgender Kriterien überprüft: Glaubwürdigkeit, Angemessenheit, Prüffähigkeit und die Beweisbarkeit (Guba und Lincoln 1981). Der Begriff der Glaubwürdigkeit meint dabei, inwieweit die Ergebnisse der Studie aus Sicht der interviewten Personen korrekt sind. Dies wurde erst in den nachfolgenden Abschnitten der Studie, an denen die Doktorandin nicht mehr beteiligt war, durch Triangulation der Methoden überprüft. Entsprechend war beim Expertenworkshop an dem Ergebnisse aus Interviews und Fokusgruppen präsentiert wurden eine Angehörigenvertretung anwesend. Angemessenheit, also die Anwendbarkeit der Ergebnisse in anderen Kontexten, wurde durch detaillierte Beschreibung der Ergebnisse sichergestellt. Dadurch wird es anderen Forschern ermöglicht diese nachvollziehen und zu entscheiden, inwiefern die Ergebnisse der Studie in anderen Kontexten einsetzbar sind. Die Gewährleistung der Prüffähigkeit geschah durch die Einhaltung fester Prozedere und Regeln. So waren die Interviews durch Leitfäden einheitlich strukturiert. Memos und Transkription erfolgten nach im Vorfeld festgelegten Regeln. Zudem waren alle beteiligten Forscher in der Durchführung und Auswertung der Interviews geschult. Durch regelmäßige Treffen im Team wurde ein einheitliches Vorgehen bei Umsetzung und Auswertung garantiert. Eine genaue Dokumentation im gesamten Prozess sicherte die Transparenz der Ergebnisse. Alle Auswertungsschritte wurden 
regelmäßig mit einer unabhängigen, nicht am direkten Auswertungsprozess beteiligten, Person besprochen und dadurch kontrolliert. Alle Daten wurden mindestens von zwei Forschern gelesen, um Beweisbarkeit sicherzustellen (Müller et al. 2015).

\subsection{Computergestützte qualitative Datenanalyse}

Für die Dissertation wurde das für qualitative Forschungsarbeiten entwickelte Computerprogramm, MAXQDA benutzt und alle Codierungen und Memos in diesem Programm erstellt.

Das Programm ermöglichte eine gut überschaubare Verwaltung sowie eine Speicherung der Teilergebnisse in verschiedenen Versionen, wodurch der Auswertungsprozess leichter nachzuvollziehen ist (Kelle 2012). Die RetrievalFunktion, mit der man Textabschnitte durch selbst festgelegte Filter aufspüren kann, wurde beim selektiven Codieren genutzt, um Parallelen, Unterschiede oder Erweiterungen der jeweiligen Ausgangdaten, Codes oder Memos zu erkennen.

Die durch die elektronische Datenverarbeitung gestützte Analyse vereinfacht die Überprüfung der jeweiligen Überlegung, indem die passenden Textstellen im Programm gesucht werden können. Denn wenn große Textmengen schlecht verwaltet werden, besteht das Risiko, dass sich Theorien auf wenige Zitate stützen und Gegenbeweise im Textmaterial übersehen werden (Kelle 2012).

Es ist jedoch nicht zu vernachlässigen, dass die durch die computergestützte Analyse mögliche Verarbeitung größerer Datenmengen die Gefahr besteht, den Forscher zu überfordern (Kelle 2012). Kritik wird auch an dem erhöhten Risiko geübt, den Kontakt zu den Rohdaten zu verlieren, da eventuell die Beschäftigung mit den generierten Codes im Vordergrund steht (Seidel und Kelle 1995).

In der vorliegenden Studie diente das Programm hauptsächlich dazu, die Organisation und Gliederung der anfallenden Datenmengen zu erleichtern, mit dem Anspruch, die Daten intensiver und strukturierter analysieren zu können als dies ohne das Programm möglich gewesen wäre. 


\subsection{Datenschutz und Genehmigung durch die Ethik- Kommission}

Die Ethik-Kommission der Universität Göttingen und auch die entsprechenden Institutionen der Standorte in Mannheim und Hamburg bewilligten die Durchführung der Studie (Antragsnummer: 17/5/12). Das vorliegende Dissertationsvorhaben wurde der Göttinger Ethik-Kommission gesondert unter der Antragsnummer DOK_111_2013 angezeigt und von dieser genehmigt. Die Bezugspersonen, die sich zuvor telefonisch bereit erklärt hatten, erhielten vor dem Interview ein Aufklärungsgespräch durch einen geschulten Projektmitarbeiter sowie eine schriftliche ausführliche Studieninformation. Im Anschluss daran wurde das Einverständnis zur Teilnahme in einer Einwilligungsklärung eingeholt. Die Partizipation an der Studie erfolgte freiwillig. Es wurde darüber informiert, dass die Einverständniserklärung jederzeit ohne Angaben von Gründen widerrufen werden könne und ein Ausscheiden aus der Studie ohne Nachteile für den Beteiligten oder dessen Angehörigen bleibt.

Die Speicherung der Originaldaten findet nur auf Datenträgern und nicht etwa im Internet statt. Im Institut für Allgemeinmedizin werden die während der Studie erhobenen Daten in pseudonymisierter Form auf Papier und elektronischen Datenträgern gespeichert und auch nur in diesem pseudonymisierten Zustand genutzt. Die Daten werden bis zehn Jahre nach Studienende im selben Institut archiviert. 


\section{$3 \quad$ Ergebnisse}

\subsection{Beschreibung der Stichprobe}

Insgesamt wurden in 23 Interviews 27 private Bezugspersonen (4 Doppelinterviews) in 14 Pflegeheimen befragt. Anteilig wurden je 6 Interviews von Mitarbeitern des Instituts für Allgemeinmedizin der Universität HamburgEppendorf, 10 von Mitarbeitern des Zentralinstituts für seelische Gesundheit in Mannheim und 7 von Mitarbeitern des Instituts für Allgemeinmedizin der Universität Göttingen geführt. Die soziodemografischen Daten der Angehörigen sind in Tabelle 1 dargestellt. Bei $73 \%$ der Heimbewohner lag eine gesetzliche Betreuung durch die Befragten vor (eine Angabe im Demografiebogen konnte nicht gewertet werden). Existierte eine gesetzliche Betreuung, waren in $93 \%$ die befragten Angehörigen selbst als Betreuer eingesetzt. Hier wurde eine Angabe wegen fehlender Nennung nicht berücksichtigt. Die Angehörigen der befragten Bezugspersonen waren im Durchschnitt zum Zeitpunkt des Interviews 24 Monate (Spanne zwischen 3 - 90 Monate) im Pflegeheim untergebracht. Im Mittel fanden 16 Besuche der Angehörigen (Spanne 2 - 30) pro Monat im Heim statt. Zwei Angehörige machten hierzu keine Angaben. Die durchschnittlich im Heim verbrachte Zeit der Angehörigen betrug dabei rund 33 Stunden pro Monat (Spanne zwischen 5 - 180 Stunden).

Von den Heimbewohnern lebten 4 in Einrichtungen von kommunalen/ öffentlichen Trägern, 7 in privat geführten, 9 in frei-gemeinnützigen und 2 in kirchlich geführten Einrichtungen (eine fehlende Angabe). Die Pflegeheime hatten dabei minimal 52 bis maximal 257 Plätze (eine fehlende Angabe). Die Befragungen fanden in den Städten selber oder deren Umgebung statt. Dabei waren 21 der eingeschlossenen Pflegeheime in Ortschaften mit mehr als 5000 Einwohnern und 2 in Ortschaften mit weniger als 5000 Einwohnern lokalisiert.

Die Interviews wurden im Zeitraum vom 26.03.2012 bis zum 04.04.2014 geführt. Im Mittel lag die Gesprächsdauer bei rund 38 Minuten (Spanne zwischen 20 - 58 Minuten). 
Tabelle 1 Soziodemografische Angaben der befragten Angehörigen

\begin{tabular}{|c|c|c|c|c|}
\hline & $\begin{array}{c}\text { Raum } \\
\text { Hamburg }\end{array}$ & $\begin{array}{c}\text { Raum } \\
\text { Mannheim }\end{array}$ & $\begin{array}{l}\text { Raum } \\
\text { Göttingen }\end{array}$ & Insgesamt \\
\hline \multicolumn{5}{|l|}{ Alter in Jahren } \\
\hline Mittelwert & 62.2 & 62.8 & 65 & 63.3 \\
\hline Altersbereich & $52-75$ & $50-76$ & $47-81$ & $47-81$ \\
\hline Geschlecht $^{\star}$ & & & & \\
\hline Weiblich & 4 & 9 & 5 & 18 \\
\hline Männlich & 2 & 5 & 2 & 9 \\
\hline Verwandtschaftsgrad* & & & & \\
\hline Ehepartner & 1 & 2 & 2 & 5 \\
\hline Kind & 5 & 8 & 3 & 16 \\
\hline Andere & 0 & 4 & 2 & 6 \\
\hline Schulabschluss* & & & & \\
\hline $\begin{array}{l}\text { Hauptschule } \\
\text { Volksschule }\end{array}$ & 1 & 7 & 2 & 10 \\
\hline $\begin{array}{l}\text { Realschule / mittlere } \\
\text { Reife }\end{array}$ & 4 & 4 & 3 & 11 \\
\hline Abitur / Fachabitur & 1 & 1 & 1 & 3 \\
\hline Andere & 0 & 2 & 1 & 3 \\
\hline
\end{tabular}

$\mathrm{n}$ gesamt $=26, \mathrm{n}$ in Hamburg $=6, \mathrm{n}$ in Mannheim $=14, \mathrm{n}$ in Göttingen $=7$, * Zahlen absolut

Der Ergebnisteil der vorliegenden Arbeit beschreibt die Ergebnisse des offenen Codierens in den Kapiteln 5.2- 5.3 sowie die Ergebnisse des axialen und selektiven Codierens in Kapitel 5.4. Das axiale und selektive Codieren des gesamten Materials würde weit über den Rahmen einer medizinischen Doktorarbeit hinausreichen. Aus diesem Grund wurde gemeinsam mit Betreuerin und Doktormutter entschieden, den Bereich „Aufgaben der Angehörigen/ Einbezogensein in die medizinische Versorgung“ als zentrales Thema dieser Dissertation hier 
detaillierter zu untersuchen. Um die Gesamtstruktur der Ergebnisse transparent zu machen, wird im Folgenden zunächst der während des Auswertungsprozesses entstandene Codebaum dargestellt. So konnten insgesamt elf Hauptkategorien gebildet werden, die innerhalb des gesamten Projektes für alle Interviewgruppen einheitlich gebraucht wurden und sich weiter in Kategorien und Subkategorien gliedern. In der folgenden Darstellung der Ergebnisse wird nicht auf alle Hauptkategorien eingegangen, da insbesondere die Hauptkategorien „Rahmenbedingungen“, „Wahrnehmung Heim“ und „Kommunikation im Interview“ hinsichtlich der Fragestellung zur medizinischen Versorgung im Pflegeheim inhaltlich wenig Berührungspunkte boten. Die Überschriften der einzelnen Textabschnitte kennzeichnen hierbei, welcher Teil des Codesbaum gerade dargestellt wird. 


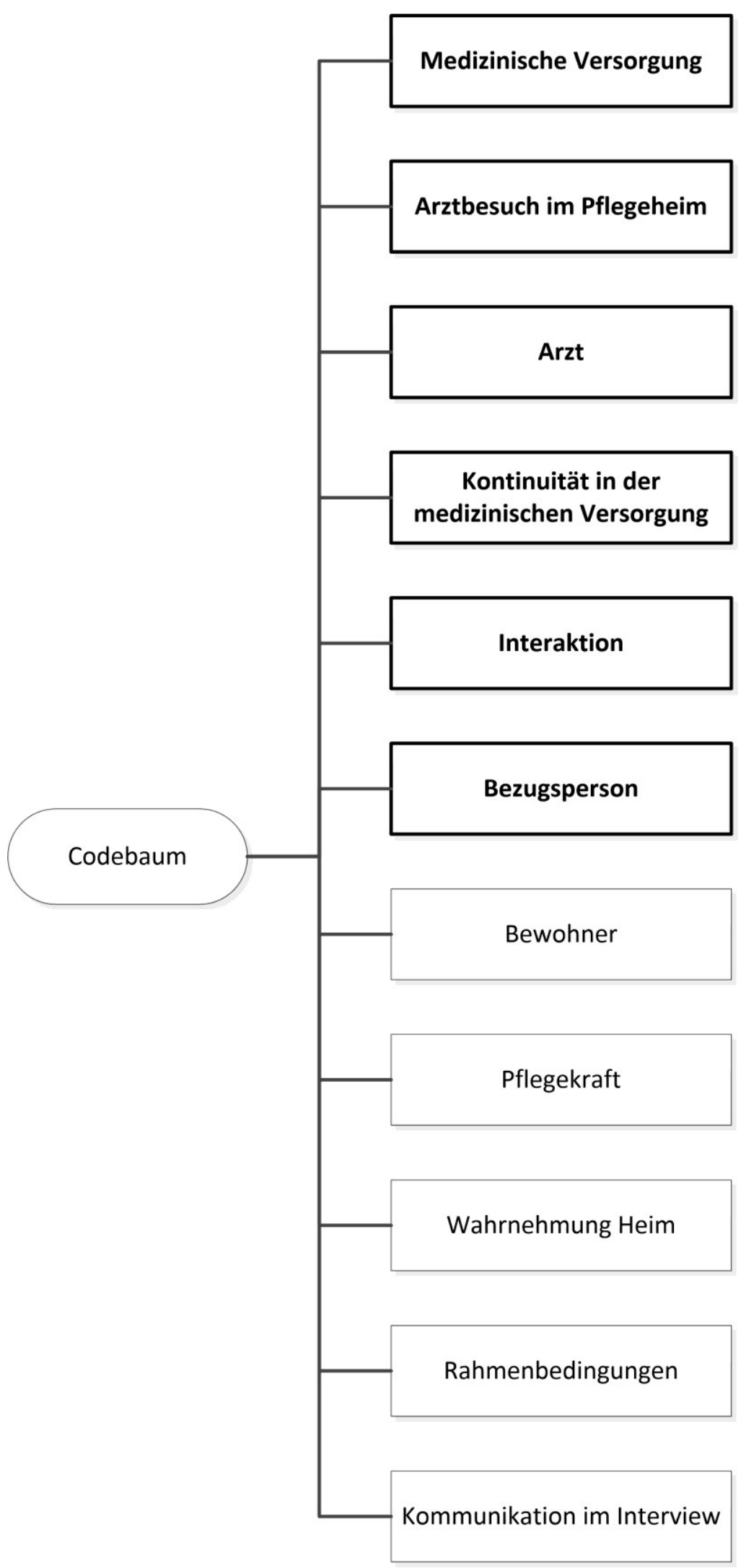

Abb. 2 Codebaum 


\subsection{Erfahrungen mit der medizinischen Versorgung}

Im Folgenden werden das Erleben der medizinischen Versorgung und die Interaktion zwischen Bezugsperson und Hausarzt sowie Pflegenden auf Basis des offenen Codierens beschrieben. Zudem erfolgt eine Darstellung der Angehörigenperspektive hinsichtlich einer Kontinuität in der medizinischen Versorgung.

\subsubsection{Arztbesuch im Pflegeheim}

Dieses Unterkapitel stellt die Ergebnisse der Hauptkategorie Arztbesuch im Pflegeheim dar. Diese Hauptkategorie wurde in die Kategorien Hausarztbesuch, Facharztbesuch, Notarztbesuch und Praxisbesuch unterteilt. Inhaltlich wird nachfolgend lediglich auf die Kategorie Hausarztbesuch eingegangen, weil dies Themenschwerpunkt der Interviews und der Auswertung war. Im Folgenden werden die Subkategorien der Kategorie Hausarztbesuch näher erläutert. Auch hierbei wird inhaltlich vor allem auf die Subkategorien eingegangen, die vorrangig von den Angehörigen in den Interviews angesprochen wurden. Die Abbildung 3 zeigt den entsprechenden Ausschnitt des Codebaums.

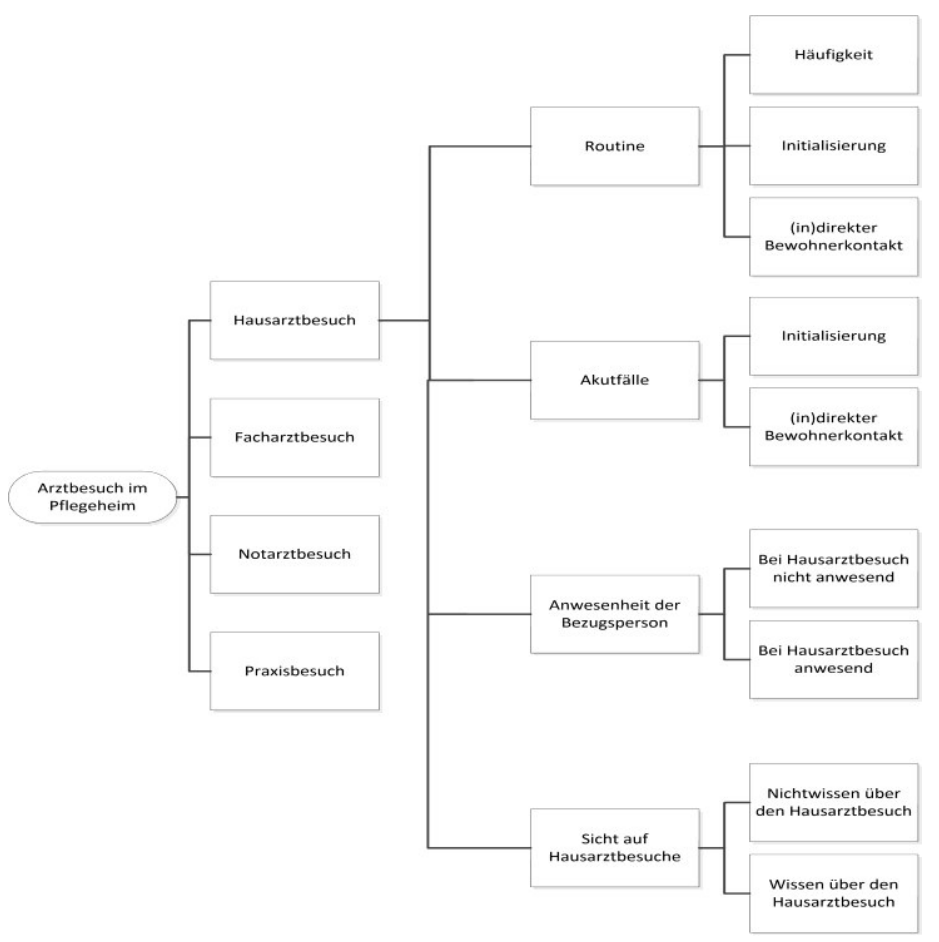




\section{Hausarztbesuch/ Routine/ Häufigkeit}

Die Kenntnis der Angehörigen über die Häufigkeit, mit der Hausarztbesuche im Pflegeheim stattfinden, stell sich sehr unterschiedlich dar. Mitunter bestehen bezüglich der ärztlichen Besuchsfrequenz keine Kenntnisse (AZ1, 164-165; BZ2, 59-60; BZ4, 48-49; BZ7, 115-116) oder es gibt nur Spekulationen (AZ2, 45-54; BZ2, 62-64; BZ10, 20). Manchmal besteht dieses Wissen auch dann nicht, wenn die Bezugspersonen täglich im Pflegeheim sind (AZ1, Demografiebogen; BZ4, 34). Sind die Bezugspersonen oft auch nicht über genaue Besuchszeiten und Häufigkeiten der Hausarztbesuche im Bilde, so haben einige doch grobe Vorstellungen über die Häufigkeit mit der Hausarztbesuche ihrer Meinung nach stattfinden. So äußern die interviewten Bezugspersonen etwa, dass Hausbesuche „regelmäßig" (AZ2, 40; AZ6, 46), „mindestens einmal im Monat" (AZ5, 91) stattfinden oder die Hausärzte „öfters ins Heim rüber“ kommen würden (BZ9, 44). Andere Angehörige können die monatlichen Besuche genau anhand der Rechnungen, die sie bekommen, nachvollziehen (BZ5, 347) oder wissen, an welchen Tagen die Ärzte einmal in der Woche im Heim sind (BZ6, 26; CZ1, 28-30; $C Z 7,72)$, weil die Besuchszeiten immer dieselben zu sein scheinen. Ein Grund für regelmäßige, wöchentliche (BZ4, 60; $C Z 7,81)$ oder 14-tägige Arztbesuche in der Einrichtung $(B Z 10,20)$ ist die Betreuung mehrerer Bewohner vor Ort (AZ6, 48, BZ10, 20; CZ7, 81).

\section{Hausarztbesuch/ Routine und Akutfälle/ Initialisierung}

Den meisten Bezugspersonen zufolge erfolgt die Initialisierung der Hausarztbesuche durch die Pflegenden (AZ1, 77; AZ5, 88-89; BZ5, 249-251; BZ9, 100-103). Insbesondere in akuten Bedarfssituationen würden die Pflegekräfte entscheiden, wann ein Arzt gerufen wird (AZ1, 188-193; AZ2, 168; AZ6, 54; BZ2, 44, 81-84; BZ4, 45; BZ9, 93; CZ1, 34; CZ5, 22, 34; CZ7, 72). Manche Interviewte konnten dazu aber auch keine Angaben machen (BZ5, 107; CZ6, 22-23). Für die Angehörigen ist ein Grund, warum die Veranlassung vor allem den Pflegekräften obliegt, dass sie selbst nicht ständig vor Ort sind.

„Na ich sag mal die Pflegekraft, die kriegt ja eher schon mit, wenn irgendwas bei meiner Mutter nicht stimmt [...] kriegt die eher mit als ich, weil die sehen sie ja viel, viel länger am Tag, ja und das, das übernimmt dann die Pflegekraft den [...] Hausarzt anzurufen." 
Als weitere Ursache wird angeführt, dass die Bewohner einen Arztbedarf mitunter nicht selbst kommunizieren können $(C Z 5,20)$. Tatsächlich wird in den Interviews nur einmal erwähnt, dass eine Bewohnerin ihre Arztbesuche selbstständig organisiert (BZ10, 20). In diesem Fall werden die Pflegekräfte nur in Akutsituationen in die Entscheidung, einen Arzt anzufordern, involviert (BZ10-12). Ansonsten wird eher auf die Angehörige zurückgegriffen, da die Bewohnerin selbstbestimmt leben möchte.

„Also meine Mutter will das halt nicht ganz aus der Hand geben, sie will da autark bleiben [...]. Von daher will sie das auch mitbestimmen, ja und wenn sie merkt, dass das gar nicht funktioniert, dann ruft sie meine Schwester an und meine [...] Schwester organisiert das dann. Also da hat das Pflegepersonal eigentlich gar nix mit zu tun."

(BZ10, 23-26)

Angehörige veranlassen die Hausarztbesuche teilweise selbst (BZ8, 18; BZ9, 46, CZ3, 42). Mitunter wird ihnen diese Aufgabe direkt von den Pflegenden übertragen (AZ3, 97-102). In den vorliegenden Fällen scheint dies darin begründet zu sein, dass die Bezugspersonen ein gutes Verhältnis zum Hausarzt haben, hier insbesondere, weil sie selbst dort Patienten sind (AZ3, 34-36; BZ8, 17-18; BZ9, 53). So schildert die Tochter einer Bewohnerin, dass sie den Hausarzt zum Teil bei ihren eigenen Arztterminen um einen Hausbesuch bei ihrer Mutter bitten würde und dieser dem Wunsch dann auch nachkommt (AZ3, 34-36). In einem anderen Fall übernimmt die Bezugsperson die Initialisierung durch direktes Aufsuchen der Arztpraxis, um einen Hausarztbesuch zu beschleunigen $(C Z 3,42)$.

\section{Hausarztbesuch/ Routine/ (in)direkter Bewohnerkontakt}

Die Schilderungen der Bezugspersonen über Abläufe und den Kontakt zum Bewohner bei Hausarztbesuchen im Pflegeheim sind unterschiedlich. So gibt es Hausärzte, die dabei jedes Mal direkten Bewohnerkontakt pflegen (CZ7, 72). Andere Hausärzte sind zwar regelmäßig im Heim, führen aber bisweilen nur eine Kurvenvisite mit den Pflegenden durch, um zu klären, ob ein unmittelbarer Kontakt zum Bewohner nötig ist (AZ6, 222; BZ10, 20).

„ICh glaube, dass sie [Hausärztin] einmal wöchentlich hingeht [...], nur dass sie vielleicht nicht immer mit ihm [Bewohner] spricht, aber mit den Schwestern. Sie erkundigt sich dann [...] wie es ihm geht und so, ob irgendwas anfällt." 
Dementsprechend sind die Beschreibungen der Abläufe der Hausarztbesuche ebenfalls unterschiedlich. So gibt es Angehörige, die von regelmäßigen Blutentnahmen und körperlichen Untersuchungen berichten.

„Ja so alle paar Wochen kriegt sie Blut abgenommen. Dann wird sie untersucht von Kopf bis Fuß."

$(B Z 6,46)$

Andere Bezugspersonen meinen, der Arzt würde bisweilen nur kurz mit dem Bewohner reden, aber dabei nicht immer eine körperliche Untersuchung durchführen (BZ1, 158).

Hausarztbesuch/ Anwesenheit der Bezugsperson/ bei Hausbesuch nicht anwesend

Die Kenntnisse über den Hausarztbesuch hingen stark davon ab, inwieweit die Bezugspersonen bereits in Hausbesuche involviert waren. Von den interviewten Angehörigen berichteten 10 Personen, dass sie bereits bei Hausarztbesuchen anwesend gewesen wären, wohingegen 13 eine Anwesenheit ihrerseits verneinten. Es wurden unterschiedliche Gründe für eine bisherige Nichtanwesenheit angeführt. So schilderten einige Angehörige, dass sie es bislang nicht als notwendig empfunden hätten, bei den Hausbesuchen dabei zu sein. Entweder, weil bei der Bewohnerin ihrer Meinung nach keine akuten gesundheitlichen Beschwerden bestünden (BZ3, 40), oder weil eine Kommunikation mit dem Arzt bislang über andere Wege stattfand (siehe Kap. 5.3.1 Interaktion zwischen Hausarzt und Bezugsperson).

„[...] ich war noch nicht im Pflegeheim, wo er [Hausarzt] eben auch da war. Das [...] hat noch nicht gepasst, war jetzt auch nicht erforderlich, weil die Sachen, die wir abklären müssten, das machen wir entweder telefonisch oder eben über meine Nachbarin oder wir gehen zu ihm."

$(C Z 5,10)$

Dass die medizinische Versorgung bisher nach Ansicht der Bezugsperson gut funktioniert hat, wird ebenfalls als Grund angegeben noch nie bei einem Arztbesuch dabei gewesen zu sein (BZ8, 21-26). Auch wenn Angehörige sich etwa durch Berichte des Bewohner hinreichend informiert fühlen, wird eine direkte Anwesenheit bisweilen nicht als erforderlich empfunden (BZ1, 18). Auch die konträren Besuchszeiten von Angehörigen und Hausärzten (BZ1, 12; BZ7, 81-82; 
CZ2, 50, 56) und bisweilen kurzen Heimbesuche der Hausärzte waren für andere Angehörige ein Grund, bisher noch keinen Arztbesuch miterlebt zu haben.

„Ich habe ihn [den Hausarzt] da im Heim nie gesehen. Der ist mal da und dann zack, zack, zack da rein, da rein und zack ist er wieder weg."

$(A Z 5,79)$

Weiterhin handhaben die Hausärzte ihre Besuche zuweilen flexibel, was es für die Angehörigen schwierig macht, den Arzt im Heim anzutreffen.

"[...] ich war in der Zeit dann da, immer als Arzt 1 sagte ich komme in der Zeit von bis, das heißt, ich hab mich hier vier Stunden aufgehalten. Herr Arzt 1 kam nicht und er kam abends um halb acht [...]"

$(\mathrm{CZ4}, 28)$

Hausarztbesuch/ Anwesenheit der Bezugspersonen/ bei Hausarztbesuch anwesend

Eine direkte Anwesenheit der Bezugspersonen bei einem Hausarztbesuch des Bewohners (AZ3, 58; AZ4, 68) oder sonstiger Kontakt zum Arzt im Heim (AZ5, 215-219; BZ9, 93), findet bisweilen eher ungeplant statt, wenn beide Akteure zufällig zur selben Zeit im Heim sind. Insbesondere wenn von Angehörigenseite Gesprächsbedarf besteht, wird ein Treffen mit dem Arzt im Pflegeheim aber auch bewusst herbeigeführt (BZ7, 62-66).

"[...] wenn ich es ganz, ganz, ganz für bedürftig halte hier zu sein, rufe ich in der Praxis an [...] und dann sagen mir die Schwestern: „Es ist geplant von bis". Dann versuche ich in der Zeit hier zu sein, weil ich gerne Arzt 1 natürlich selbst [...] daraufhin ansprechen möchte."

$(C Z 4,28)$

Feste Besuchszeiten der Hausärzte erleichtern hierbei eine Anwesenheit der Angehörigen.

"Ich bin [...] eigentlich immer dabei, weil ich ja [...] weiß, der kommt am Dienstag. Also normal am Montag- oder Dienstagabend zwischen halb fünf und fünf."

$(\mathrm{CZ7}, 72)$ 
Hausarztbesuch/ Sicht auf Hausarztbesuche/ Nichtwissen über den Hausarztbesuch

So beruhen die Beschreibungen der Hausbesuche auch oft auf Vermutungen oder verschiedene Punkte, etwa Ablauf, Anlass und genaue Besuchszeiten der Arztbesuche, sind den Angehörigen nicht bekannt.

Die Unkenntnis der genauen Besuchszeiten der Hausärzte (AZ5, 219; BZ4, 90-95; CZ4, 28; CZ5, 18) ist mitunter darin begründet, dass diese teilweise kurzfristig $(C Z 5,18)$ oder nicht immer zur selben Zeit (BZ4, 90-95) stattfinden würden.

Hausarztbesuch/ Sicht auf Hausarztbesuche/ Nichtwissen über den Hausarztbesuch/ Bewohner berichtet nicht über den Hausarztbesuch

Weiterhin verhindert in vielen Fällen die kognitive Einschränkung der Bewohner, dass diese ihren Angehörigen von stattgefundenen Hausarztbesuchen erzählen (AZ1, 173; BZ3, 60-64; BZ4, 50-53; BZ5, 48-51; BZ6, 49-50; BZ9, 53-60). Andererseits sind die Hausbesuche mitunter auch lediglich kein oder kaum Gesprächsthema zwischen Bezugsperson und Bewohner (AZ4, 93-96; BZ1, 26).

Hausarztbesuch/ Sicht auf Hausarztbesuche/ Wissen über den Hausarztbesuch/ Berichte des Bewohners

Es gibt jedoch auch Heimbewohner, die ihre Angehörigen über stattgefundene Hausbesuche $(A Z 3,58)$ und deren Verläufe (BZ2, 98; CZ4, 30) unterrichten, wenn auch nicht immer unbedingt detailliert (AZ6, 144-146).

Hausarztbesuch/ Sicht auf Hausarztbesuche/ Wissen über den Hausarztbesuch/ Informierung durch die Pflegekräfte

Häufiger erhalten die Angehörigen von den Pflegekräften im Heim Auskunft über die Hausarztbesuche. So informieren sich die Bezugspersonen bisweilen aktiv bei den Pflegekräften darüber (BZ3, 43-46; BZ6, 46). Manche Pflegekräfte würden diesbezüglich jedoch auch von sich aus berichten (BZ3, 41-42, 57; BZ6, 60-62; BZ9, 93; CZ1, 32, 68; CZ2, 50). Dementsprechend äußert ein Ehemann, dass er insbesondere dann nachfrage, wenn die Pflegekräfte vergessen hätten, ihm vom Hausbesuch zu erzählen (CZ2, 46). 
Hausarztbesuch/ Sicht auf Hausarztbesuche/ Wissen und Nichtwissen über den Hausarztbesuch/ Informierung durch den Hausarzt

Angehörige berichten auch vereinzelt davon, direkt vom Arzt über erfolgte Hausbesuche in Kenntnis gesetzt zu werden.

„Dann sagt er mir auch manchmal Bescheid, ruft an: „Ich war bei ihrer Mutter."

$(A Z 3,58)$

Hier ist nicht erkennbar, in welchen Situationen der Arzt die Angehörige über seine Besuche informiert. In anderen Fällen unterrichtet der Arzt die Angehörigen telefonisch über eine nötige Krankenhauseinweisung des Bewohners (AZ6, 86-88; $B Z 9,38)$.

Zusammenfassend lässt sich feststellen, dass die Angehörigen der Heimbewohner in sehr unterschiedlichem Ausmaß über die Hausarztbesuche im Heim informiert sind. So werden die Hausarztbesuche in den meisten Fällen von den Pflegekräften und nur selten von den Angehörigen veranlasst. Mehr als die Hälfte der Befragten verneint zudem, jemals bei einem Hausarztbesuch anwesend gewesen zu sein. Die Gründe hierfür sind vielfältig. Mitunter wird eine Anwesenheit von den Angehörigen nicht als nötig empfunden. Zudem haben beide Akteure bisweilen konträre Besuchszeiten oder Kommunikation findet über andere Wege statt. Entsprechend erhalten die Bezugspersonen Informationen über die Hausarztbesuche in unterschiedlichem Umfang von den Bewohnern selbst, häufig jedoch auch durch die Pflegekräfte und in Ausnahmefällen direkt durch den Hausarzt.

\subsubsection{Sichtweisen und Erfahrungen bezüglich der Ärzte}

Nachfolgend werden die Ergebnisse des offenen Codierens der Hauptkategorie „Sichtweisen und Erfahrungen bezüglich der Ärzte“ beschrieben. Innerhalb dieser Hauptkategorie ergaben sich die drei Kategorien Rollenerwartung Außenperspektive, Fachärzte und Zahnärzte sowie andere Therapeuten. Außenperspektive bedeutet hier, die Rollenerwartung an den Arzt von außen, in diesem Fall durch die Angehörigen. Im Folgenden wird ausschließlich auf die Kategorie Rollenerwartung Außenperspektive eingegangen, da sich die Auswertung auf die medizinische Versorgung durch die Hausärzte konzentriert. Die Abbildung 4 zeigt 
den zugehörigen Ausschnitt des Codebaums mit den Subkategorien, auf die im Anschluss näher eingegangen wird.

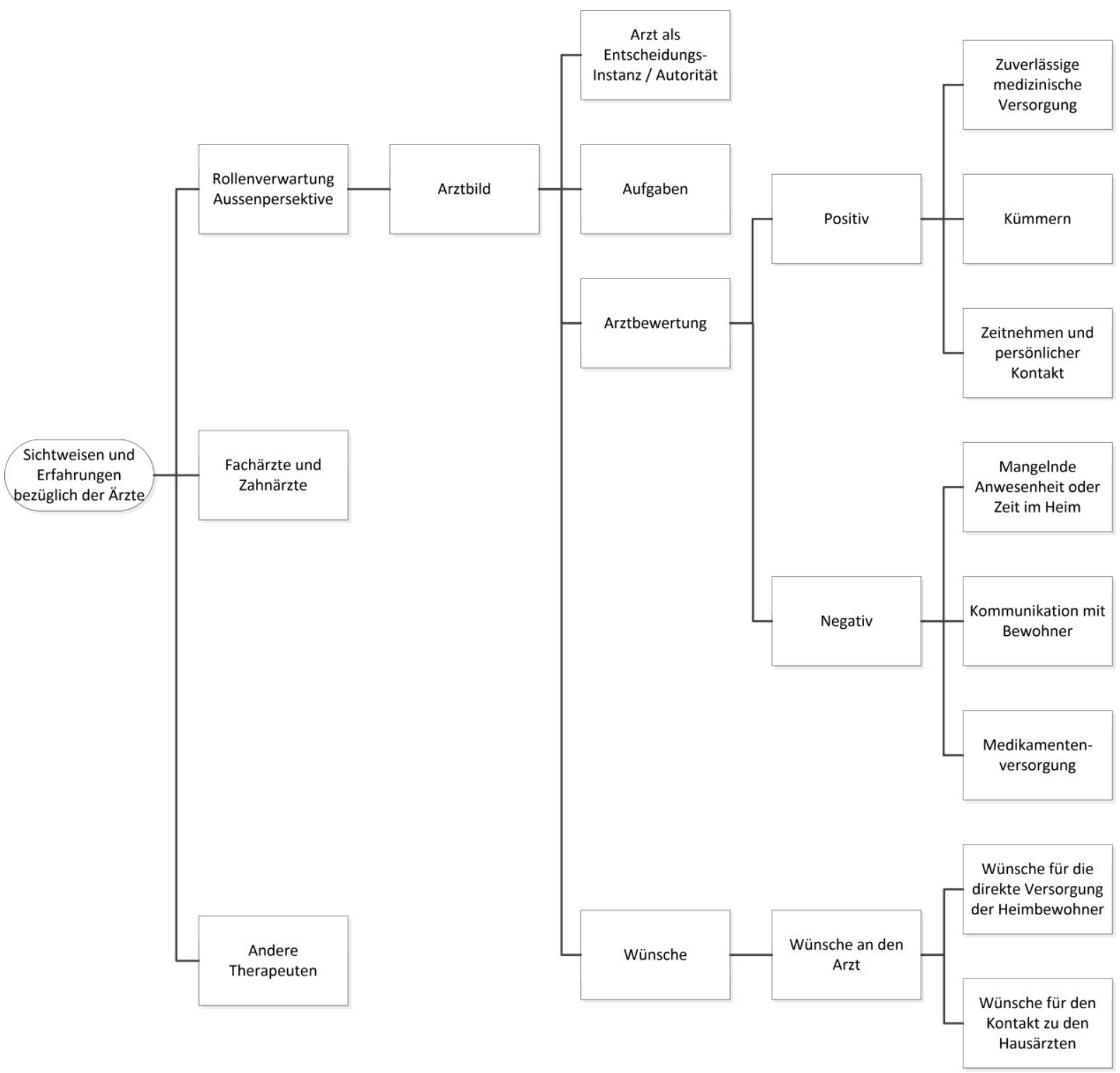

Abb. 4 Hauptkategorie Sichtweisen und Erfahrungen bezüglich der Ärzte

Arzt/ Rollenerwartung Außenperspektive/ Arztbild/ Arzt als Entscheidungsinstanz/

$\underline{\text { Autorität }}$

Von einigen Bezugspersonen werden die Ärzte klar als Autoritäten und Entscheidungsinstanzen in der medizinischen Versorgung wahrgenommen.

So etwa gibt es Angehörige, die meinen, der Arzt solle die Entscheidungen treffen und die Bewohner lediglich kurz darüber informieren. 
„Der [Hausarzt] soll [...] sagen, pass auf [...], du brauchst die und die Tablette, ob die hilft oder nicht hilft. [...] Das wollen glaube ich viele ältere Leute. Die wollen nicht so [...] [ei]ne genaue Auflistung haben oder einen Bericht haben da drüber. Die wollen nur ganz kurz informiert werden."

Auch in der Beschreibung der Zusammenarbeit mit den Pflegenden zeigt sich, dass die Ärzte hier als die Entscheidungsträger angesehen werden. Hierauf wird jedoch noch näher im Kapitel 5.3 Erfahrungen mit der Zusammenarbeit zwischen Ärzten und Pflegekräften eingegangen.

„da hat es geheißen plötzlich, die Frau Dr. Arzt 1 hat es erlaubt, sie [Pflegekräfte] dürfen das machen“

$(\mathrm{BZ4}, 68)$

Die Vorstellung der Autorität der Ärzte geht mitunter soweit, dass sich Bezugspersonen nicht vorstellen können, was sich ein Arzt im Pflegeheim noch wünschen könnte, da ohnehin alles deren Willen entsprechend geschehen würde.

„Kann ich jetzt gar nix sagen, [...] was der [Hausarzt] sich wünschen könnte. Ich denk doch immer halt, dass das halt so geht, was er verordnet und was er sagt, dass [...] das dann auch ausgeführt wird."

(BZ2, 112)

\section{Arzt/ Rollenerwartung Außenperspektive/ Arztbild/ Aufgaben}

Die Bezugspersonen stellen vor allem das Sicherstellen der Medikamentenverordnung und -versorgung als ärztliche Aufgaben in den Vordergrund (AZ3, 109-112; AZ4, 124; AZ5, 158-159; BZ2, 78; BZ7, 101; CZ7, 96).

„Na ich denk mal die Aufgaben der Ärzte werden in dem Moment nur sein, dass die gucken und irgendwie die Medikamente verordnen oder irgendwelche [...] anderen Dinge, die medizinische Verordnung [...]. Das ist aber auch das Einzige."

$(\mathrm{CZ6}, 30)$ 
Arzt/ Rollenerwartung Außenperspektive/ Arztbild/ Arztbewertung

Die Subkategorie Arztbewertung ließ sich aufgliedern in positive und negative Einschätzungen des Arztes. Im Anschluss werden zunächst die Punkte dargestellt, die von den Angehörigen positiv wahrgenommen wurden.

Arzt/ Rollenerwartung Außenperspektive/ Arztbild/ Arztbewertung/ positiv/ zuverlässige medizinische Versorgung

Teil einer guten Versorgung ist für die Angehörigen, dass die Hausärzte regelmäßig im Heim präsent sind und verlässlich eine ärztliche Betreuung erfolgt (BZ9, 44-46).

Auch hätten regelmäßige Hausarztbesuche einen gewissen Placeboeffekt und würden dem Bewohner ein gutes Gefühl vermitteln.

"[...] sie [Bewohnerin] war ja immer schon so, sie muss ganz oft zum Arzt. Allein schon, dass die den regelmäßig sieht, hab ich das Gefühl, reicht eigentlich schon für vieles."

$(B Z 1,52)$

Dass Hausärzte zuverlässig und zeitnah im Bedarfsfall zur Stelle sind, ist ein wichtiges Kriterium für eine positive Bewertung der Arztes (AZ3, 102; AZ6, 46; BZ9, 46).

„[...] wenn was ist [...], ist er [Hausarzt], immer gleich da“

(BZ2, 60-62)

Entsprechend wird es positiv erlebt, wenn die Bezugspersonen das Gefühl haben, der Arzt würde bei Krankheit des Bewohners häufiger ins Heim kommen und Untersuchungen durchführen. So berichtet die Tochter einer Bewohnerin, bei der Behandlung ihrer Mutter wäre es „schon so gewesen, dass die [Hausärzte] da dran waren" und die Hausärztin hier dementsprechend öfter im Heim gewesen sei $(A Z 6,78)$.

Ebenfalls positiv gewertet wird es, wenn der Arzt bei einer Gesundheitsveränderung des Bewohners schnell und dem Empfinden der Angehörigen nach mit der richtigen Entscheidung reagiert. 
„Und er [Hausarzt] hat das auch mit der Blutarmut rausgefunden. Ja der hat die [Bewohnerin] untersucht und hat [eine] Blutuntersuchung gemacht und da hat er uns am nächsten Tag abends gleich um halb sechs da angerufen. Hat gesagt, [...] sie muss sofort in die Klinik. Und wäre sie oben [in ihrem ehemaligen Haus] gewesen, will ich Ihnen sagen, wäre sie nicht mehr da."

$(B Z 9,38)$

Arzt/ Rollenerwartung Außenperspektive/ Arztbild/ Arztbewertung/ positiv/ Kümmern

Besonders positiv wird die ärztliche Betreuung beurteilt, wenn die Bezugspersonen das Gefühl haben, die Hausärzte würden sich um die Bewohner „kümmern“ (AZ1, 233; AZ6, 46; CZ2, 68). Dies beinhaltet etwa, dass Ärzte Engagement hinsichtlich der Behandlung des Bewohners zeigen, etwa auf eine Mobilisierung der Bewohnerin drängen $(\mathrm{CZ2}, 68)$. Aber auch regelmäßige Besuche beim Bewohner können als Aspekt des Kümmerns angesehen werden.

„Aber er [Hausarzt] guckt auch so immer rein und [...] und guckt nach ihr [Bewohnerin]"

(AZ2, 32)

Arzt/ Rollenerwartung Außenperspektive/ Arztbild/ Arztbewertung/ positiv/ Zeitnehmen und persönlicher Kontakt

Im Umgang mit den Bewohnern wird es positiv wahrgenommen, wenn der Arzt sich bei seinen Besuchen Zeit für den Bewohner nimmt (AZ3, 84-90; BZ2, 68).

Von den Angehörigen wird die Wichtigkeit eines persönlichen Kontaktes hervorgehoben und Gespräche zwischen Arzt und Bewohner über rein Medizinisches hinaus sehr positiv gewertet.

„[...] der Arzt 1, der hat mit meinem Vater ein super Verhältnis gehabt, weil er nie über seine Krankheit gesprochen hat. Der war sehr geschickt. Der konnte mit alten Leuten sehr gut umgehen. Der hat da drüber gesprochen, weil mein Vater Handwerker ist, dass er zu Hause was umgebaut hat und hat er ihn um Rat gefragt. [...] Wenn der da sich eine Viertelstunde Zeit genommen hat, dann ging es vielleicht eine Minute um die Krankheit, [...] er [Bewohner] hatte das Glück gehabt, dass er da eigentlich nie krank war, so richtig krank. [...] Der [Arzt 2] erzählt viel, aber nur über seine Krankheit und deswegen ist das kein guter Arzt für ihn [Bewohner]."

$(C Z 7,51)$ 
Es wird gewürdigt, wenn der Arzt Bewohner und Bezugsperson Gehör schenkt (AZ2, 64; BZ2, 68), oder auch persönliche Bemerkungen, etwa eine Gratulation zum Geburtstag der Bewohnerin, macht (BZ2, 44).

„Also es ist nicht [...] nur so dieses sture: „Äh, so du willst [...] eine Grippeimpfung haben? Jetzt hast du die Spritze und nun habe ich mein Soll erfüllt und Tschüss!", ne? Nein, das ist es nicht bei ihm. Also er bleibt auch noch mal fünf Minuten sitzen [...] und dann sitzt er da [...] als wenn er die größte Zeit der Welt hat. [...] Dann nimmt er sich die Zeit und dann ist das gut."

(AZ3, 84-90)

Eine private Bezugsperson geht so weit zu sagen, die Zuwendung des Arztes wäre beim Besuch das Wesentliche und die eigentliche medizinische Versorgung sekundär.

„Die Zuwendung das ist das Wichtigste. Ja also meine Adoptivtante hat ihren Hausarzt über alles geliebt und wenn der eine halbe Stunde für sie Zeit hatte, das war das Beste. Also diese blöden Tabletten, die kann man sowieso nicht schlucken, so aus ihrer Sicht ja. Die wollte sie eigentlich gar nicht haben."

(CZ3, 110)

Arzt/ Rollenerwartung Außenperspektive/ Arztbild/ Arztbewertung/ negativ

Es gibt ebenfalls Aspekte, die zu einer negativen Bewertung des Arztes führen. Am häufigsten bezieht sich die Kritik der Bezugspersonen an der hausärztlichen Betreuung im Heim auf seltene, beziehungsweise zeitlich lediglich kurze Besuche. Mitunter wird auch Kritik an der Medikamentenversorgung geübt.

Arzt/ Rollenerwartung Außenperspektive/ Arztbild/ Arztbewertung/ negativ/ mangelnde Anwesenheit oder Zeit im Heim

Eine mangelhafte Präsenz im Pflegheim wird den Hausärzten seitens der Bezugspersonen unterstellt, wenn sie selbst den Hausarzt noch nie dort angetroffen haben $(\mathrm{CZ6}, 45)$.

„Einen Arzt habe ich im Pflegeheim noch nie gesehen.“

(AZ5, 79)

Nicht nur mangelnde Präsenz des Hausarztes im Heim, sondern auch lediglich kurze Besuche werden negativ gewertet $(B Z 10,84)$. 
Einige private Bezugspersonen zeigen hierfür ein gewisses Verständnis unter der Annahme, dass die Heimbesuche nicht gut vergütet würden und mit Aufwand verbunden wären (AZ5, 211-213). Auch, dass Hausärzte wenig Zeit hätten, wird angeführt.

„Die Hausarztbesuche sind sehr kurz [...] logischerweise, weil weiß ich nicht, Hausärzte haben vielleicht auch nicht unbegrenzte Zeit. "

$(C Z 4,6)$

Nichtsdestotrotz äußern Angehörige, wenn sie die Besuche beim Bewohner als zu kurz empfinden, Kritik teilweise deutlich und fordern, dass Hausärzte mehr Zeit für die Heimbesuche aufbringen.

„[...] der Arzt müsste auch viel mehr Zeit aufwenden, wenn er sagt, ich betreue Menschen im Heim. [...] es ist alles easy zu sagen, ich gehe dahin, ich habe da [...] fünf Oma $X Y$ [...] toll ich kann das super abrechnen. Arztbesuch [...] hingefahren, was weiß ich Ziffer blablabla und [...] war ja prima, alles in einer Einrichtung und Tschüss und nach Hause. [...] ist ein guter Verdienst."

$(\mathrm{CZ4}, 20)$

Außerdem glaubt die Tochter einer Bewohnerin, diese könne im Gespräch mit der Hausärztin nicht alles ansprechen. So würde die Hausärztin keinen Raum für persönliche Bemerkungen lassen und sich zu wenig Zeit für den Kontakt mit der Bewohnerin nehmen.

„Ich glaube, wenn meine Mutter eine etwas längere Ansprache hätte und auch wenn mal nachgefragt wird, wie geht es ihnen und was ist da und was ist da mit und dann würde sie [Bewohnerin] auch bestimmt andere Dinge erzählen und so hat sie das Gefühl, die Ärztin ist sowieso gehetzt und wenn ich jetzt auch noch mit dem bisschen komme, das schaffe ich auch alleine und das ist schade eigentlich."

(BZ10, 84)

Dem ähnelnd meint ein Angehöriger, sein Vater würde die Hausarztbesuche als sehr kurz empfinden und das Gefühl haben, der Arzt höre ihm nicht zu.

„Ja also mein Vater würde jetzt sagen, der [Hausarzt] kommt rein ist gleich wieder draußen und wenn ich ihm was sage, dann hört er nicht zu und ich sage, die hören beide nicht zu."

$(C Z 7,67)$ 
Arzt/ Rollenerwartung Außenperspektive/ Arztbild/ Arztbewertung/ negativ/ Kommunikation mit Bewohner

Kritik am direkten Umgang mit dem Bewohner wird selten geäußert. Eine Angehörige nimmt Anstoß daran, dass Ärzte häufig nicht versuchen würden, mit den Bewohnern auf Augenhöhe zu kommunizieren.

„[...] oft werden sie behandelt wie kleine Kinder, die Ansprache ist auch manchmal wie bei kleinen Kindern, aber das sind erwachsene Menschen."

(BZ10, 152)

Zudem würden Bedürfnisse der Bewohner nicht immer ernst genug genommen und etwa Beschwerden über Schmerzen zwar angehört, aber ärztlicherseits nichts dagegen unternommen.

„[...] da hab ich manchmal den Eindruck, dass man das sich anhört, das abnickt und sich aber nix verändert [...]"

(BZ10, 90)

Arzt/ Rollenerwartung Außenperspektive/ Arztbild/ Arztbewertung/ negativ/ Medikamentenversorgung

Ein weiterer Aspekt, der bei einigen Angehörigen zur Kritik am ärztlichen Handeln führt, ist die medikamentöse Versorgung der Bewohner. Mitunter wird den Ärzten vorgeworfen, sie würden die Bewohner mit Medikamenten überversorgen, um diese ruhig zu stellen.

So gibt es Vorwürfe, der Hausarzt würde in Absprache mit den Pflegekräften zu viele Medikamente verordnen, um ein häufiges Klingeln des Bewohners zu unterbinden $(C Z 4,6)$. Überdies wird unterstellt, der Hausarzt würde sich nicht mit der Höhe der gegebenen Medikamentendosis auseinandersetzen, was zu einem schlechteren Gesundheitszustand des Bewohners führen würde.

„Sie [Bewohnerin] kriegt dann ein Schmerzpflaster [...] $50 \mathrm{mg}$. Das ist so etwas was [...] morphiumartig ist und was noch höher dosiert ist. [...] Das kriegt sie dann von dem Arzt verschrieben, der sich, gehe ich mal von aus, nicht damit auseinandersetzt. Wie viel ist schon 4 x 40 Medikament 1 [...]. Teilweise dosieren die dann also das Schmerzpflaster plus [...] die 
Höchstmenge Medikament 1. Daraus resultiert, dass meine Mutter phantasiert [...]. Dass sie Krabbeltiere überall in diesem Raum sieht. Dass sie Menschen sieht, die nicht da sind."

$(C Z 4,10)$

Andere Bezugspersonen beobachten bei einigen Heimbewohnern plötzliche Apathie und Verhaltensveränderungen und äußern deshalb Vermutungen hinsichtlich einer medikamentösen Überversorgung.

BZ1: „Dann bin ich doch manchmal sehr erstaunt über den Zustand, der sich von heute auf morgen radikal ändert und dann denke ich oft: „Vielleicht [...] kriegen die doch ein bissel zu viel Medikamente." [...] kann sein, dass ich da Ärzten mit zu nahe trete [...] aber manchmal denke ich mir: „Ach die gibt ja gar nix mehr von sich, die sitzt ja nur noch lethargisch da."

BZ2: „Wird sie ruhig gestellt.“

(BZ3,153-154)

Ähnlich berichtet ein Angehöriger, seine Frau würde aufgrund früheren aggressiven Verhaltens medikamentös ruhiggestellt (AZ5, 43). Er äußert zwar keine direkte Kritik an diesem Vorgehen, schildert jedoch, dass Unterhaltungen mit seiner Frau nicht mehr richtig möglich seien und sieht die Gründe hierfür sowohl in der zunehmenden dementiellen Erkrankung der Bewohnerin, als auch in der Medikation.

„Naja und meine Frau [...] in der ersten Zeit konnte man sich ja mit ihr normal unterhalten, nicht? Das ist ja noch vor gut einem Jahr gewesen. Jedenfalls [...] ist das dann immer schlechter geworden. Demenz oder was immer da ist, hat zugenommen. Und [...] die wird ja auch ruhig gestellt über die Medikamente und die meiste Zeit schläft sie ja und sitzt da im Rollstuhl im Aufenthaltsraum."

$(A Z 5,11)$

Die Organisation der Medikamentenversorgung wird bisweilen auch kritisch wahrgenommen. So berichten Angehörige davon, dass die medikamentöse Versorgung unterbrochen wurde, weil sich der Hausarzt längere Zeit im Urlaub befand (BZ5, 88) oder die Zuständigkeiten zwischen den Fachärzten bei der Verschreibung nicht klar geregelt waren. 
„Und nun waren die [Medikamentenname] alle und die sollten nun aufgeschrieben werden von der Hausärztin und das hat sie nicht gemacht. Und dann musste ich zum Urologen [...] er war jedenfalls im Urlaub und ich kriegte nichts. Und ja, das war schon sehr schlimm. Und dann [...] habe ich noch mal da in der Praxis angerufen. Und da hat sie [Hausärztin] uns doch tatsächlich nach langem Theater ein Rezept fertig gemacht."

$(A Z 1,43)$

In anderen Fällen wird Unzufriedenheit mit dem Ablauf der Medikamentenverschreibung deutlich. So wird kritisiert, dass die Medikamentenverordnungen bisweilen willkürlich seien und sehr häufig geändert würden.

„es wird abgesetzt, es wird hochdosiert, es wird [...] andere Medikation aufgeschrieben, verschrieben, berechnet [...] mir erscheint das hier alles sehr willkürlich."

$(\mathrm{CZ4}, 24)$

Kritik wird jedoch nicht nur an der Medikamentenverschreibung geübt, sondern auch wenn durch die Medikamenteneinnahme notwendige Kontrolluntersuchungen des Hausarztes aus Angehörigensicht nicht häufig genug stattfinden. So wird kritisiert, dass diese mangelhafte Versorgung möglicherweise gesundheitlich negative Folgen für den Bewohner haben könne (BZ10, 34).

"[...] sie "[Bewohnerin] ist [Medikamentenname]-Patientin, sie [Hausärztin] soll das mal überprüfen [...] und da ist ein[...] Problem. Es ist auch schon vorgekommen, dass sie [Hausärztin] das vergessen hat oder dass es einfach zu viel war und sie denkt, das ist nicht so wichtig. Ja und meine Mutter kriegt das aber erst mit, wenn sie nicht kommt. Das heißt, sie wartet und wartet und wartet und dann kommt sie nicht und das ist nicht so angenehm. Also da würde ich mir eine Verbesserung wünschen für sie, ja. “

(BZ10, 20)

\section{Arzt/ Rollenerwartung Außenperspektive/ Arztbild/ Wünsche}

Innerhalb der Subkategorie Wünsche wurden von den Angehörigen Wünsche geäußert, die sich auf die direkte Versorgung des Bewohners beziehen und solche, die den Kontakt mit der Bezugsperson betreffen. Im Anschluss wird zunächst auf die Wünsche hinsichtlich Bewohnerversorgung eingegangen. 
Arzt/ Rollenerwartung Außenperspektive/ Arztbild/ Wünsche/ Wünsche an den Arzt/ Wünsche für die direkte Versorgung der Heimbewohner

Von den Medizinern wird sich bezüglich der Hausbesuche beim Bewohner gewünscht, dass der Arzt sich „kümmert" und Besuche sowohl regelmäßig (BZ1, 90), als auch zuverlässig bei Bedarf stattfinden (BZ5, 265-266). Desweiteren sollten diese dabei immer mit Bewohnerkontakt erfolgen, also über eine reine Kurvenvisite hinaus gehen.

„Und dann gucken die [Hausärzte] natürlich manchmal auch nur in die Akte [...], weil da steht alles drinnen. Aber für so einen Menschen ist das natürlich auch schick, wenn der "Guten Tag" sagt. Weil [...] dann weiß man ja, dass der sich gekümmert hat."

(AZ6, 222-224)

Ebenfalls wird der Wunsch nach längeren Heimbesuchen des Arztes, verbunden mit einem persönlicheren Kontakt zum Bewohner, formuliert (AZ5, 259).

„Also von den Hausärzten würde ich mir tatsächlich eine etwas persönlichere Ansprache wünschen und auch vielleicht eine längere Verweildauer."

(BZ10, 84)

Zudem wird geäußert, dass Ärzte Patientenbeschwerden, etwa im Bezug auf Schmerzen, mitunter ernster nehmen (BZ10, 90) und gegebenenfalls Therapiealternativen in Betracht ziehen sollten (BZ10, 128). Vereinzelt wird sich eine gute Schmerztherapie für den Bewohner gewünscht (AZ1, 233). Andere Angehörige formulieren globaler den Wunsch nach einer liebevollen und guten Versorgung, ohne dies genauer zu definieren (AZ1, 239).

Arzt/ Rollenerwartung Außenperspektive/ Arztbild/ Wünsche/ Wünsche an den $\underline{\text { Arzt/ }}$

Wünsche für den Kontakt zu Hausärzten

Überdies bestehen bei den Angehörigen auch Anliegen für den eigenen Kontakt mit den Hausärzten der Bewohner, welche sich vor allem auf die Kommunikation beziehen. So wird von Bezugspersonen, die die gesetzliche Betreuung innehaben, der Wunsch geäußert, dass ein Informationsaustausch auch vom Arzt ausgehend 
erfolgt. Etwa wünschen sich Angehörige vereinzelt, dass sie über die Medikation der Bewohnerin besser in Kenntnis gesetzt (BZ3, 190-197) und auch nach Routinebesuchen informiert werden.

„[...] man kann ja mal kurz anrufen und sagen: „ICh war bei der Mutter, es ist alles in Ordnung."

$(B Z 3,150)$

Andere möchten auch über anstehende Besuche benachrichtigt werden, um die Möglichkeit zu erhalten, ein Gespräch mit Arzt, Pflegekraft, Bewohner und Angehörigem zu führen.

„Ja, dass er [Hausarzt] mal anruft und sagt: „Ich bin dann da und da auf dem Zimmer und [...] dass man da in Gegenwart der Patientin oder [...] Pflegeperson [...] mal sich ausspricht oder so was, nicht?" (AZ5, 253-259)

Gleichzeitig wird hier jedoch an der Umsetzbarkeit durch vermutete mangelnde Ressourcen des Hausarztes gezweifelt.

„Ja, dass er das persönliche Gespräch mal sucht, von sich aus. Nicht? Aber Gott, was sollen sie noch alles, die haben ja nicht so viel Zeit."

$(A Z 5,253-257)$

Zusammenfassend ist herauszustellen, dass Ärzte von den Bezugspersonen als Autoritäten und Entscheidungsinstanzen in der medizinischen Versorgung wahrgenommen werden. Positiv wird es von den Angehörigen gewertet, wenn Hausärzte regelmäßig im Heim präsent sind und auch im Bedarfsfall zuverlässig eine medizinische Versorgung gewährleisten. Ebenfalls wird ein etwas persönlicherer Kontakt bei Hausarztbesuchen beim Bewohner geschätzt und dass Hausärzte sich während dieser Besuche Zeit nehmen. Entsprechend werden mangelhafte Präsenz der Hausärzte im Heim kritisiert und auch lediglich kurze Besuche negativ bewertet. Zudem unterstellen einige Angehörigen den Hausärzten eine medikamentöse Überversorgung der Bewohner, um diese ruhigzustellen. Als Wunsch formulieren die Angehörigen, wenn dies nicht der Fall ist, dass Hausarztbesuche im Heim regelmäßig und stets mit Bewohnerkontakt 
stattfinden. Zudem wird der Wunsch nach einem persönlicheren Kontakt zum Bewohner zum Ausdruck gebracht. Für den eigenen Kontakt zum Hausarzt wünschen sich die Angehörigen bisweilen mehr Informationsaustausch vom Arzt ausgehend.

\subsubsection{Kontinuität in der medizinischen Versorgung}

Nachfolgend wird die Hauptkategorie Kontinuität in der medizinischen Versorgung dargestellt. Innerhalb dieser Kategorie ergaben sich die Subkategorien Hausarztbeibehaltung, Hausarztwechsel und Sicht auf die Kontinuität in der medizinischen Versorgung. Abbildung 5 stellt den zugehörigen Ausschnitt des Codebaums dar.

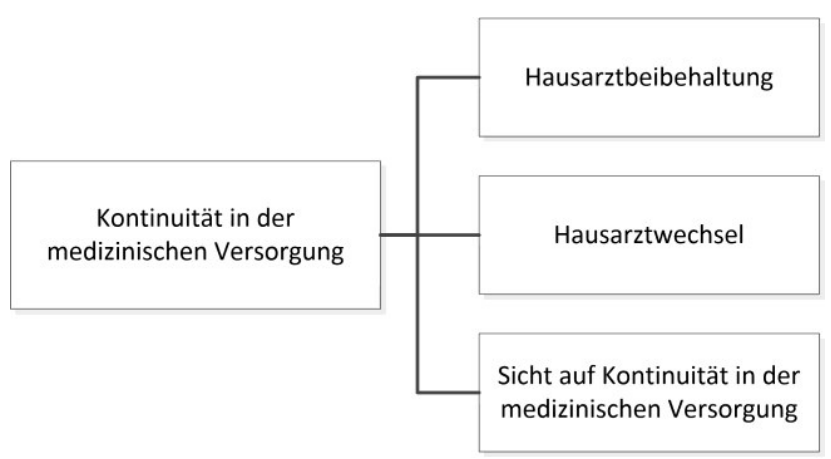

Abb. 5 Hauptkategorie Kontinuität in der medizinischen Versorgung

Die Frage, ob die Heimbewohner eine Kontinuität in der ärztlichen Versorgung nach ihrem Heimeinzug erlebten, ergab unterschiedliche Ergebnisse, auch hinsichtlich der Betrachtungsweisen der Bezugspersonen, ob sie diese Kontinuität als wünschenswert ansahen. In den Interviews zeigte sich, dass der Hausarzt nach Heimeinzug des Bewohners etwa genauso häufig beibehalten wurde $(n=9)$, wie er wechselte $(n=12)$. In zwei Fällen wurden hierzu keine Aussagen gemacht.

\section{$\underline{\text { Kontinuität in der medizinischen Versorgung/ Hausarztwechsel }}$}

Fand ein Hausarztwechsel statt, gaben die Angehörigen dafür verschiedene Gründe an. So waren es häufig Faktoren, auf die Bewohner und private Bezugspersonen keinen Einfluss hatten, etwa, weil der vorherige Hausarzt in Rente gegangen (AZ2, 38; BZ8, 7-14; BZ9, 39-40) oder verstorben war (CZ7, 4451). Andere ehemalige Hausärzte würden generell keine Heimbesuche durchführen (BZ3, 40; CZ5, 15-18) oder hätten durch den Heimeinzug einen zu 
langen Anfahrtsweg (BZ2, 50-52). Mitunter beschäftigten die Heime auch Vertragsärzte, wodurch ein Arztwechsel stattfand (AZ4, 54-60, BZ7, 53-60).

Ein aktiver Wechsel des Hausarztes auf Wunsch der interviewten Bezugspersonen und der Bewohner wurde in zwei Fällen beschrieben und erfolgte dann aufgrund einer Unzufriedenheit mit der bisherigen ärztlichen Versorgung. So wurden als Gründe Probleme mit der medikamentösen Versorgung, nicht stattfindenden Kontrolluntersuchungen ( $A Z 1$, 65) oder eine unzuverlässige Besuchsfrequenz des Hausarztes angegeben.

"[...] eine Zeit ist er gekommen, aber dann lief das nicht so, dann ist er später gekommen oder gar nicht. Naja und dann haben wir gesagt, ne das geht einfach nicht, weil er [Bewohner] ja erst aus dem Krankenhaus gekommen war, nicht. Also da musste ja jemand da sein, wenn was ist, ne."

$(C Z 1,50)$

Auch in anderen Fällen waren die Bezugspersonen aktiv an der Wahl des neuen Arztes beteiligt. Mehrere Bezugspersonen berichteten, der Kontakt zum neuen Hausarzt wäre darüber entstanden, dass dieser bereits andere Bewohner im Heim betreute (AZ1, 67; BZ2, 52; CZ5, 18). Mitunter wird die ärztliche Betreuung des Bewohners auch vom Hausarzt der Angehörigen übernommen (BZ9, 33-38).

Kontinuität in der medizinischen Versorgung/ Sicht auf Kontinuität in der medizinischen Versorgung

Die generellen Einstellungen zur Kontinuität in der medizinischen Versorgung gingen in den Interviews auseinander. Ein Grund, warum das Beibehalten des bisherigen Hausarztes von den Bezugspersonen gut geheißen wurde, war dessen Kenntnis der Krankengeschichte des Bewohners (AZ5, 83). Das Wissen um die enge Bindung des Bewohners an den bisherigen Hausarzt spielte eine wichtige Rolle in den Bewertungen. So wurde in diesen Fällen eine Kontinuität in der Versorgung auch von den Angehörigen als bedeutend angesehen (BZ1, 92) oder aber die eigene Meinung über einen Hausarztwechsel trotz Unzufriedenheit mit der ärztlichen Betreuung zurückgestellt (BZ10, 46).

„Für meine Mutter ist es halt so, sie ist eine bekannte Ärztin. Die hatte sie schon immer, die hatte auch schon mein Vater, der jetzt seit zehn Jahren 
nicht mehr lebt und [...] sie will halt [...] diesen Bezug, diesen persönlichen Bezug, den sie ja doch empfindet, nicht hergeben ja."

$(\mathrm{BZ10}, 46)$

In anderen Fällen wurde zwar anerkannt, dass der vorherige Hausarzt den Bewohner länger kannte, jedoch mehr Wert auf andere Dinge gelegt, etwa dass die Praxis des neuen Arztes dicht neben dem Pflegeheim lag (BZ5, 267-269). Hier erschien der ehemalige Hausarzt durchaus ersetzbar, solange der neue Hausarzt ebenfalls durch seine Persönlichkeit überzeugen konnte.

„Nein da kommt bestimmt auch ein anderer Arzt dann, wenn er sympathisch ist ja."

(BZ5, 271-273)

Situationen, in denen ein Arztwechsel stattgefunden hatte, wurden bisweilen als unproblematisch erlebt (BZ1, 167-170). Im Gegensatz zu einem langjähren Hausarzt-Patienten-Verhältnis beschreiben Angehörige jedoch einen Unterschied im Kontakt zum Hausarzt bei Arztwechsel, da die Erfahrung im gegenseitigen Umgang fehle.

„[...] wir sind eigentlich so damit zufrieden. Das ist natürlich nicht so ein Kontakt, als wenn ich zu meiner Hausärztin gehe. Ja, das ist natürlich was anderes, weil [...] die [...] Erfahrung hat man einfach nicht."

$(C Z 5,18)$

Zusammenfassend lässt sich feststellen, dass ein Hausarztwechsel bei Heimeinzug der pflegebedürftigen Angehörigen häufig stattfand, ohne dass die privaten Bezugspersonen und deren Angehörige Einfluss darauf hatten. Nur in wenigen Fällen wurde ein Hausarztwechsel aktiv aufgrund einer Unzufriedenheit mit der ärztlichen Versorgung herbeigeführt. Die Einstellungen der Bezugspersonen hinsichtlich einer Kontinuität in der medizinischen Versorgung waren unterschiedlich. Einige hießen ein Fortbestehen der hausärztlichen Betreuung aufgrund der Beziehung des Bewohners zum bisherigen Hausarzt und dessen Kenntnis der Krankengeschichte gut. In anderen Fällen wurde ein Hausarztwechsel jedoch auch als unproblematisch erlebt. 


\subsection{Interaktion}

Die Hauptkategorie Interaktion gliedert sich in verschiedene Kategorien, die jeweils eine Wechselbeziehung zwischen zwei Akteursgruppen im Pflegeheim beschreiben. Im Folgenden wird zunächst auf die Kategorie der Interaktion zwischen Bezugsperson und Hausarzt eingegangen. Anschließend wird in einem weiteren Unterkapitel die Interaktion zwischen Bezugsperson und Pflegekräften und schließlich noch die Interaktion zwischen Ärzten und Pflegekräften dargestellt. Die Abbildung 6 stellt den Ausschnitt des Codebaums zur Hauptkategorie Interaktion dar.

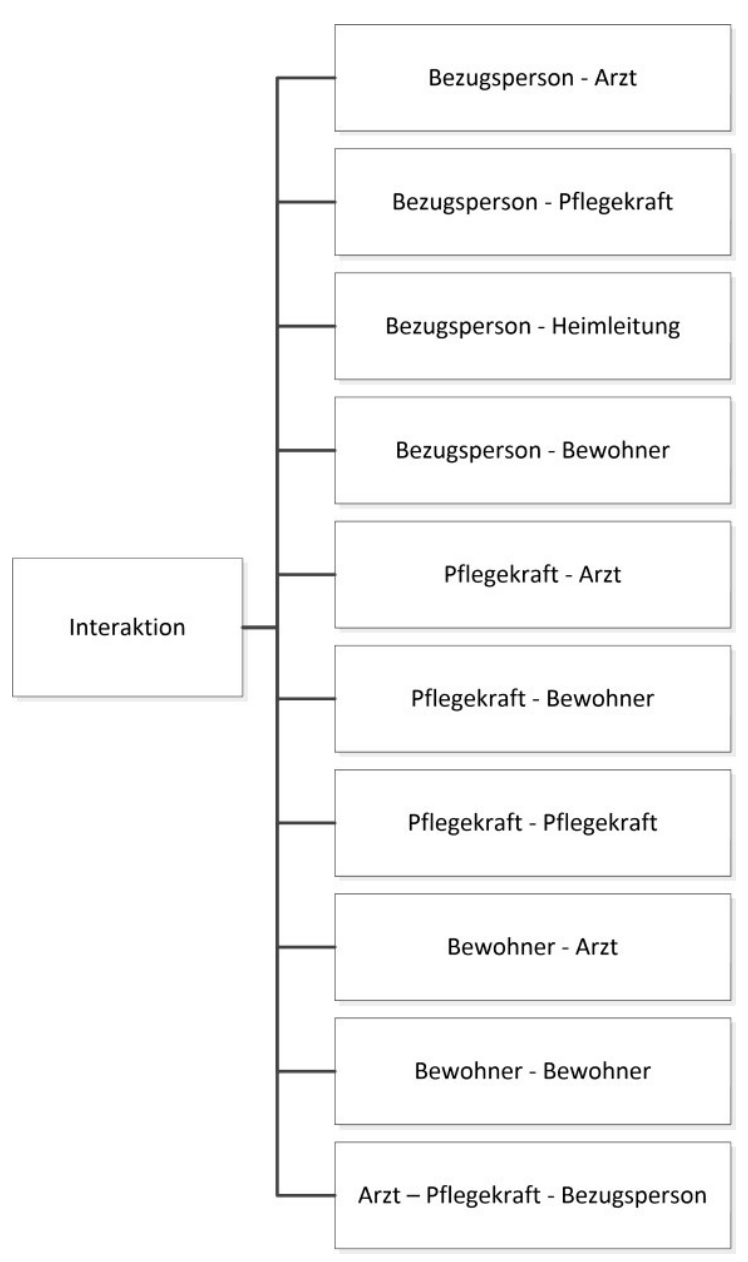

Abb. 6 Hauptkategorie Interaktion 


\subsubsection{Interaktion zwischen Bezugsperson und Arzt}

Innerhalb der Kategorie Interaktion zwischen Bezugsperson und Hausarzt ergaben sich die Subkategorien Themen der Interaktion, Kommunikationswege, gelungene/ misslungene Interaktion, Beziehung, Vertrauen-Vertrauensverhältnis, sowie Interaktion mit dem Facharzt. Auf die Interaktion mit Fachärzten wird im Folgenden nicht näher eingegangen, da sich die Auswertung auf die hausärztliche Versorgung konzentriert. In der Abbildung 7 ist der entsprechende Ausschnitt des Codebaums dargestellt.

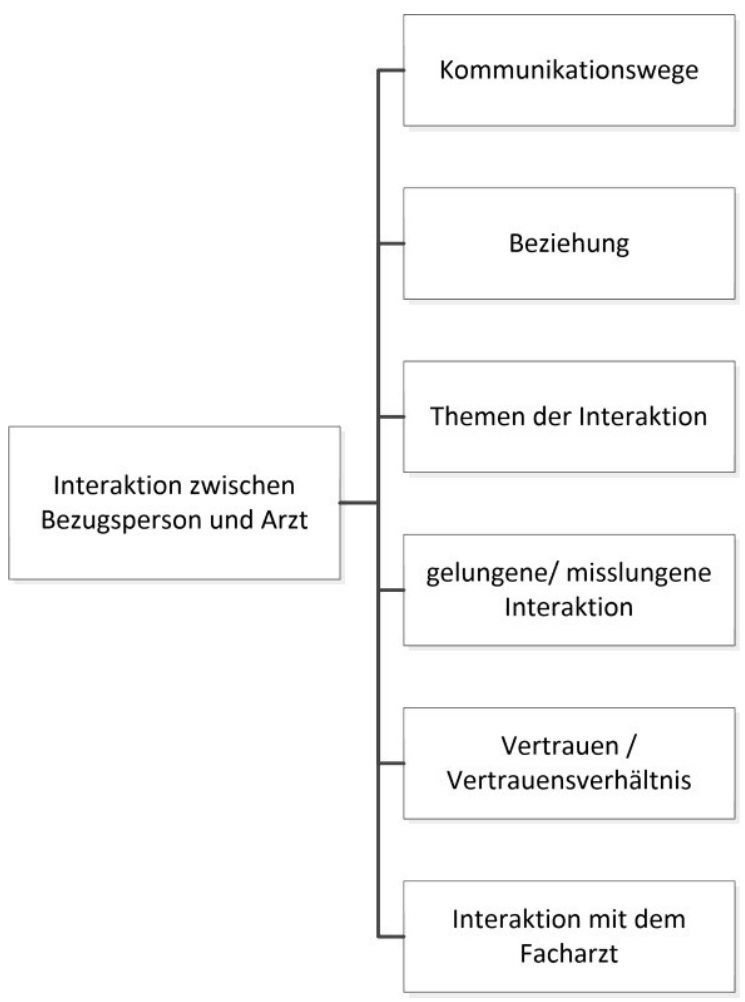

Abb. 7 Kategorie Interaktion zwischen Bezugsperson und Arzt

\section{Interaktion/ Bezugsperson-Arzt/ Kommunikationswege}

Eine Interaktion zwischen Hausarzt und Bezugsperson findet über verschiedene Wege statt. Wie bereits im Kapitel 5.2.1 Arztbesuch im Pflegeheim dargestellt, kommunizieren Hausarzt und Angehörigen mitunter direkt im Heim miteinander. Zum Teil haben Ärzte und Bezugspersonen jedoch unterschiedliche Besuchszeiten im Pflegeheim (siehe Kapitel 5.2.1 Arztbesuch im Pflegeheim). Entsprechend suchen die Bezugspersonen auch den Kontakt in der Praxis des Hausarztes oder sind sogar selbst Patienten dort. Wenn Kontakt gewünscht wird, formulieren die 
Angehörigen, dass hierfür Eigeninitiative ihrerseits nötig wäre (AZ4, 320; $B Z 1,18$; BZ7, 142-146).

\section{Interaktion/ Bezugsperson-Arzt/ Beziehung}

Von den interviewten Bezugspersonen hatten einige denselben Hausarzt wie ihre pflegebedürftigen Angehörigen, was eine Kommunikation zwischen Hausarzt und Angehörigen erleichtert (AZ3, 37-40, AZ6, 46; BZ1, 140-142; BZ4, 59; BZ5, 57-58, BZ8, 17-18, CZ2, 44-46).

Interaktion/ Bezugsperson-Arzt/ Kommunikationswege/ Kommunikation in Praxis des Arztes

So wird denn auch bei eigenen Hausarztbesuchen der Bezugspersonen über den Bewohner gesprochen (BZ6, 38-40), mal indem sich der Angehörige aktiv über den Gesundheitszustand des Bewohners erkundigt (AZ3, 60-62, BZ1, 140; BZ9, 53, CZ2, 50) oder auch vom Arzt ausgehend (AZ3, 34-36; BZ5, 57-58).

„Wir sind schon im Gespräch, weil wir auch Patienten bei ihr sind, dann spricht man auch mal drüber. Dann fragt sie [Hausärztin] auch mal: „Wie geht es dem Vater?" Und dann erzählt man mal [...], also es ist schon guter Kontakt ja. Ich will sagen, das ist schon so richtig hausarztmäßig wie man sich es vorstellt."

$(B Z 4,59)$

Diese Situation wird mitunter als sehr zweckdienlich angesehen, da sowohl eigene Angelegenheiten als auch die der Angehörigen gleichzeitig bei einem Besuch beim Hausarzt geklärt werden können.

„Und dann kann man eben auch mal das eine oder andere gleich mit besprechen. Dann bin ich eben zweimal da sozusagen. Das ist also ganz praktisch."

$(A Z 6,46)$

So werden solche Situationen bisweilen auch genutzt, um den Hausarzt um einen Besuch bei der Bewohnerin zu bitten (AZ3, 34).

Aber auch wenn der Hausarzt nicht derselbe ist, suchen Angehörige den Arzt teilweise bei Gesprächsbedarf in dessen Praxis auf. Dies geschieht insbesondere 
dann, wenn der Hausarzt nicht im Heim angetroffen wird (AZ5, 101-103).

„Und wenn ich noch irgendwas wissen möchte, dann fahr ich zu ihr [Hausärztin] hin. [...] spreche ich mit ihr [...]. Denn sie wohnt ja hier am Platz 1. Das ist für mich ja kein Weg. [...] Und donnerstags und meist wenn sie Dienstag kommt und ich Donnerstag sowieso zum Markt hinfahre [...], dann [...] schaue ich da gleich rein."

$(\mathrm{CZ1}, 58-62)$

Interaktion/ Bezugsperson-Arzt/ Kommunikationswege/ Kommunikation über Dritte In anderen Fällen erfolgt eine Kommunikation regelmäßig über Dritte. So gibt es Bezugspersonen, die persönliche Kontakte zu Mitarbeitern des Hausarztes nutzen, um Informationen über Hausbesuche bei der Bewohnerin zu erhalten (CZ5, 22). Dieser Zugang wird umgekehrt dann auch vom Hausarzt selbst verwendet (CZ5, 54). Die medizinischen Fachangestellten sind zusätzliche Informationsquellen, selbst wenn durchaus direkter Kontakt zum Hausarzt besteht (AZ2, 322-327, CZ4, 36).

\section{Interaktion/ Bezugsperson-Arzt/ Themen der Interaktion}

Die Themen der Interaktion zwischen Arzt und Bezugsperson drehen sich um die Versorgung und den Gesundheitszustand des Bewohners, etwa den letzten Hausarztbesuch $(\mathrm{CZ2}, 50)$. Der Hausarzt hilft den Angehörigen bisweilen durch Erklärungen, den Bewohner und seine Krankheit besser zu verstehen (BZ9, 126).

„Also der Arzt sagt mir, die [Bewohnerin] versteht [...]. Meine Frau zum Beispiel versteht, was gesagt wird, aber sie kann sich nicht äußern, also keine Rückantwort geben."

Andere Gesprächsinhalte sind etwa Medikation (AZ5, 173-177, CZ4, 12), Mobilisierung $(C Z 5,52)$ und Inhalte der Patientenverfügung (AZ5, 105-109, BZ10, 70). 
Interaktion/Bezugsperson-Arzt/ gelungene/misslungene Interaktion/ gelungene Interaktion

Für eine gelungene Interaktion ist es den Angehörigen wichtig, dass der Hausarzt auf ihre Anliegen, etwa Bitten nach Heimbesuchen oder Anpassungen der Behandlung der Bewohnerin, eingeht (AZ5, 61-63, CZ3, 62).

„Oder wenn ich dann hingehe, sage: „Herr Doktor, gehen sie mal hin, irgendwie das und das gefällt mir nicht." [...] fährt er eigentlich nächsten Tag her und kommt. [...] Also [...] das läuft auch gut [...]."

(AZ3, 34-36)

Zudem wird es positiv wahrgenommen, wenn der Hausarzt das Gespräch mit den Bezugspersonen über die medizinische Versorgung des Bewohners sucht und sich die Angehörigen dahingehend involviert fühlen (AZ3, 20).

„Er [Hausarzt] hat mich angerufen und dann haben wir da kurz drüber gesprochen und da hatte ich aber auch schon hier mit dem Schmerzarzt gesprochen, mit Arzt 2. Und dann war das alles in Ordnung, sagt er: „Dann kann ich das alles so machen?" „Ja“, sage ich, "Herr Doktor, können wir alles so machen." [...] Und d[a]nn ist das gut.

$(A Z 3,73-80)$

Vor allem wird es positiv gewertet, wenn sich die Angehörigen vom Hausarzt hinreichend informiert fühlen.

„[...] es geht ja darum, findet eine Kommunikation zwischen dem Pflegepersonal, den Hausärzten und [...] den Angehörigen statt. [...] Und das findet statt. Und das wird auch rückgekoppelt [...]“

(AZ6, 96-98)

Interaktion/Bezugsperson-Arzt/ gelungene/misslungene Interaktion/ misslungene $\underline{\text { Interaktion }}$

Bezugspersonen kritisieren die Interaktion mit dem Hausarzt, wenn dieser bei der Behandlung der Bewohner nicht so agiert, wie die Bezugsperson es sich wünscht. Etwa werten es die Angehörigen negativ, wenn der Arzt ihren Bitten hinsichtlich der medizinischen Versorgung nicht nachkommt. 
"Und da hat sie [Hausärztin] gesagt: „Ach, die Haut, das ist nicht so schlimm. Das machen wir nächstes Mal." Und damit war das dann erledigt, das Ganze."

$(\mathrm{AZ1}, 195)$

Mitunter handeln die Bezugspersonen dann am Hausarzt vorbei, ziehen etwa eigenmächtig andere Fachärzte hinzu, was wiederum das Verhältnis zwischen Hausarzt und Angehörigen belastet.

„[...] da war die Ärztin schon sauer, dass ich da hingefahren war." (AZ1, 51)

In anderen Situationen fühlen sich die Angehörigen machtlos, wenn Hausärzte auf Wünsche nicht reagieren. So berichtet eine Angehörige, sie hätte in Gesprächen mit dem Hausarzt wiederholt eine niedrigere Medikation für ihre Mutter gefordert, jedoch ohne Erfolg $(C Z 4,12)$.

Unterschiedliche Vorstellungen von Hausarzt und Bezugsperson bezüglich der medizinischen Versorgung der pflegebedürftigen Verwandten können zu Konflikten zwischen beiden Akteuren führen. So gibt es Angehörige, die als Konsequenz den Hausarzt des Bewohners wechseln, weil ihren Forderungen nicht nachgekommen wird und Unzufriedenheit mit der ärztlichen Behandlung besteht.

„[...] als ich nach dem Pflaster gefragt habe, da hat sie [Hausärztin] zu mir gesagt: "Sie wollen ihre Mutter ruhig stellen." Da war ich natürlich sauer, ich meine, [...] ich habe sie immer gepflegt, ich will sie nicht ruhig stellen. Ich will, dass sie keine Schmerzen hat, mehr will ich nicht und das wussten sie hier auch alle, nicht? Also das war schon wirklich, es war nicht schön. Es war wirklich nicht schön."

$(A Z 1,65)$

Interaktion/Bezugsperson-Arzt/ Vertrauen-Vertrauensverhältnis/ Arzt als Ansprechpartner

Der Hausarzt ist für die Angehörigen Ansprechpartner in belastenden Situationen. Auch wenn die Möglichkeit, mit dem Arzt zu sprechen, noch nicht in Anspruch genommen wurde, so ist es für die Bezugspersonen doch wichtig, dass sie das Gefühl haben, ein Kontakt wäre bei Bedarf möglich. 
"Wenn dann irgendwas anliegt, dann denke ich, wird das auch kein Problem sein, dass wir uns treffen [...] da [...] bin ich mir ziemlich sicher."

Zudem wird auch die Möglichkeit geschätzt, mit dem Hausarzt über sensible Themen sprechen zu können, etwa Zweifel hinsichtlich getroffener Behandlungsentscheidungen.

„[...] mit dem kann man auch über alles sprechen. Also das muss ich schon sagen."

$(A Z 5,111)$

Wenn der Hausarzt den Bezugspersonen in schwierigen Situationen mit der Krankheit des Bewohners zur Seite steht, wird das von den Angehörigen sehr wertgeschätzt.

„Ich muss ja jetzt auch [...] mal zum Arzt gehen, da sprechen wir also auch schon über die Mutti und ich bin auch froh, muss ich sagen, dass wir sie als Hausärztin haben, weil, sie hat uns schon unterstützt, als die Mama so böse wurde durch die Demenz."

$(B Z 6,40)$

Aus anderen Schilderungen geht hervor, dass Gespräche mit dem Hausarzt den Angehörigen helfen mit dem krankheitsbedingten Verhalten des Bewohners umzugehen (BZ5, 342). Eine Angehörige berichtet, sie könne den Arzt bei Gesprächsbedarf ihrerseits immer anrufen.

„[....] wenn ich irgendwie was auf dem Herzen hab und ruf ihn an, krieg ich ihn immer."

(BZ2, 44)

Zusammenfassend lässt sich feststellen, dass ein Kontakt zwischen Arzt und Bezugsperson, sowohl im Pflegeheim, als auch in der Praxis des Hausarztes stattfindet. Zum Teil haben die Bezugspersonen und deren pflegebedürftige Angehörige denselben Hausarzt. In solchen Fällen ist die Kommunikation zwischen Arzt und Bezugsperson erleichtert, weil auch bei deren eigenen Hausarztbesuchen über den pflegebedürftigen Angehörigen gesprochen werden 
kann. Die Themen der Interaktion zwischen beiden Akteuren drehen sich um die Versorgung und den Gesundheitszustand des Bewohners, etwa dessen Medikation oder Mobilisation. Zudem ist der Hausarzt für manche auch Ansprechpartner in belastenden Situationen, die Krankheit des pflegebedürftigen Angehörigen betreffend. Den Bezugspersonen ist es hierbei wichtig, dass der Hausarzt auf ihre Anliegen eingeht und sie sich hinreichend über die Versorgung des Bewohners informiert fühlen. So kommt es zu Konflikten, wenn der Arzt nicht so vorgeht, wie die Bezugspersonen es wünschen.

\subsubsection{Interaktion zwischen Bezugsperson und Pflegekraft}

Nachfolgend wird die Kategorie Interaktion zwischen Bezugsperson und Pflegekraft dargestellt. Innerhalb dieser Kategorie wurden die Subkategorien Ansprechpartner, Themen der Interaktion, Kommunikationswege, gelungene/misslungene Interaktion, Beziehung, sowie Vertrauen-Vertrauensverhältnis gebildet. Die Abbildung 8 stellt den entsprechenden Ausschnitt des Codebaums dar. Im Folgenden wird vor allem auf die Rolle der Pflegenden als Ansprechpartner, sowie die Themen der Interaktion zwischen Bezugsperson und Pflegekraft eingegangen, da diese Subkategorien Berührungspunkte mit der medizinischen Versorgung der Bewohner zeigten. 


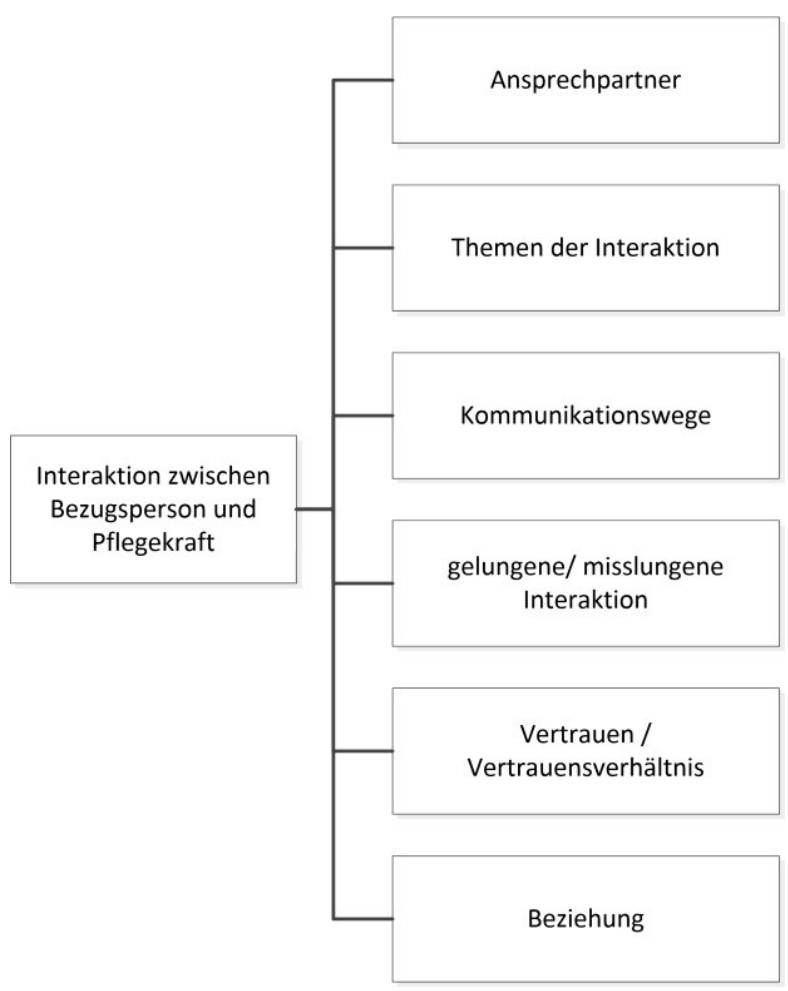

Abb. 8 Kategorie Interaktion zwischen Bezugsperson und Pflegekraft

Interaktion/ Bezugsperson-Pflegekraft/ Ansprechpartner

Die Pflegenden sind für die Bezugspersonen Hauptansprechpartner im Heim. Wie bereits erläutert, schätzen die Angehörigen auch den Hausarzt als Ansprechpartner. Dieser ist jedoch häufig nicht so einfach zugänglich, sodass eine Interaktion viel seltener, in eigenen Fällen auch gar nicht, stattfindet.

Einige Bezugspersonen berichten, ihr erster Kontakt beim Besuch im Pflegeheim wären zunächst die Pflegekräfte, um sich über besondere Vorkommnisse beim Bewohner zu informieren (AZ6, 6-10, CZ1, 69-74).

„[...] bei mir ist das einfach so, dass ich, wenn ich komme, erst mal in [...] das Pflegezimmer gehe [...], um zu erfahren, ob irgendwas Besonderes gewesen ist."

$(\mathrm{CZ} 5,2)$

In einigen Fällen gibt es Bezugspflegekräfte für die einzelnen Bewohner, die dann von den Angehörigen auch als spezielle Ansprechpartner genutzt werden (AZ2, 177-184; AZ4, 206-208; AZ6, 18). 
Bisweilen sind die Pflegekräfte der erste Ansprechpartner bei gesundheitlichen Fragen zum Bewohner und beim Hausarzt werden erst in einem zweiten Schritt Informationen eingeholt.

„Naja, ich erkundige mich schon, wenn ich keine Rückmeldung kriege [...] von den [...] Pflegern. Oder sagen wir mal so, ich erkundige mich nach, [...] wie ihr [Bewohnerin] Zustand ist jetzt [...] und dann, wenn ich da nichts Neues höre, dann rufe ich natürlich beim Hausarzt an."

$(C Z 5,36)$

Häufig übernehmen die Pflegekräfte die Rolle eines Mittlers zwischen Angehörigen und Ärzten, beispielsweise um Informationen weiterzuleiten. So werden die Pflegenden zu den Besuchszeiten bestimmter Ärzte im Heim befragt, wenn Angehörige das Gespräch mit diesen suchen (AZ6, 104-106; BZ6; 36). Darüber hinaus schildern Bezugspersonen, sie würden Beobachtungen zum Gesundheitszustand des Bewohners an die Pflegenden übermitteln, die diese dann ihrerseits an den Arzt weiterleiten (AZ5, 139-149; CZ1, 30, CZ2, 50).

Auch der Wunsch nach einem Hausarztbesuch wird mitunter an die Pflegenden gerichtet (CZ7, 72-77).

\section{Interaktion/ Bezugsperson-Pflegekraft/ Themen der Interaktion}

Die Themen der Interaktion zwischen Angehörigen und Pflegekräften drehen sich rund um den Bewohner und dessen Versorgung (AZ6, 10; BZ7, 26).

„[...] in erster Linie redet man ja mal über seinen Angehörigen [...], über die Sachen, [...] die da anstehen ja und natürlich kommen dann auch persönliche Gespräche zum Tragen [...]"

(BZ2, 20)

Besondere Vorkommnisse, auch im Hinblick auf den Gesundheitszustand des Bewohners sind Gegenstand der Kommunikation.

„[...] sie [Bewohnerin] kann nicht ständig kommunizieren und von daher frage ich einfach immer, ob irgendwas gewesen ist und grad durch den Krankenhaus[aufenthalt], den sie jetzt vorher hatte, interessiert mich natürlich, ob irgendwas Besonderes war [...]"

$(C Z 5,2-3)$ 
Auch bei Fragen zur Medikation wenden sich die Angehörigen bisweilen an die Pflegekräfte (AZ1, 221; BZ5, 86-89; BZ9, 14).

Die Pflegekräfte informieren die Angehörigen über Hausarztbesuche beim Bewohner (BZ3, 43-46; BZ6, 46; BZ9, 93; CZ1, 32, 68; CZ2, 50) und den Gesundheitszustand des Bewohners (BZ2, 12). Insbesondere in akuten Situationen gesundheitlicher Verschlechterung würden Angehörige von den Pflegekräften benachrichtigt werden (AZ6, 56-64; BZ3, 46; BZ6, 196; BZ9, 46; CZ5, 22).

„Das ist immer über die Pflege gelaufen, also die Information, was passiert ist $[\ldots]^{\prime \prime}$

$(A Z 4,146)$

Angehörige müssen mehr Eigeninitiative zeigen, wenn sie außerhalb von besonderen Vorkommnissen etwas zum Bewohner wissen möchten (AZ4, 257264; BZ3, 46, BZ5, 252-254; CZ5, 5-6).

„Normal ist es so, wenn die jetzt die Tabletten umstellen oder so, sagen die mir das schon, die Schwestern, aber erst wenn ich frag. [...] Wenn ich nachfrage, hat sich irgendwas geändert oder so."

(BZ6, 60-62)

Zusammenfassend lässt sich feststellen, dass die Pflegekräfte für die Bezugspersonen Hauptansprechpartner im Heim sind. Bisweilen agieren die Pflegenden als Mittler zwischen Angehörigen und Arzt, etwa um Informationen weiterzuleiten. Die Themen der Interaktion zwischen Pflegekräften und Angehörigen drehen sich rund um den Bewohner. So informieren Pflegekräfte über Hausarztbesuche, den Gesundheitszustand des Bewohners, sowie besondere Vorkommnisse.

\subsubsection{Interaktion zwischen Pflegekraft und Arzt}

In diesem Unterkapitel wird die Kategorie Interaktion zwischen Pflegekraft und Arzt dargestellt. Diese Kategorie wurde in die Subkategorien Nichtwissen/ Vermutungen zur Interaktion, Kommunikationswege, Themen der Interaktion, gelungene/ misslungene Interaktion sowie Beziehung unterteilt. In der Abbildung 9 wird der entsprechende Ausschnitt des Codebaums dargestellt. 


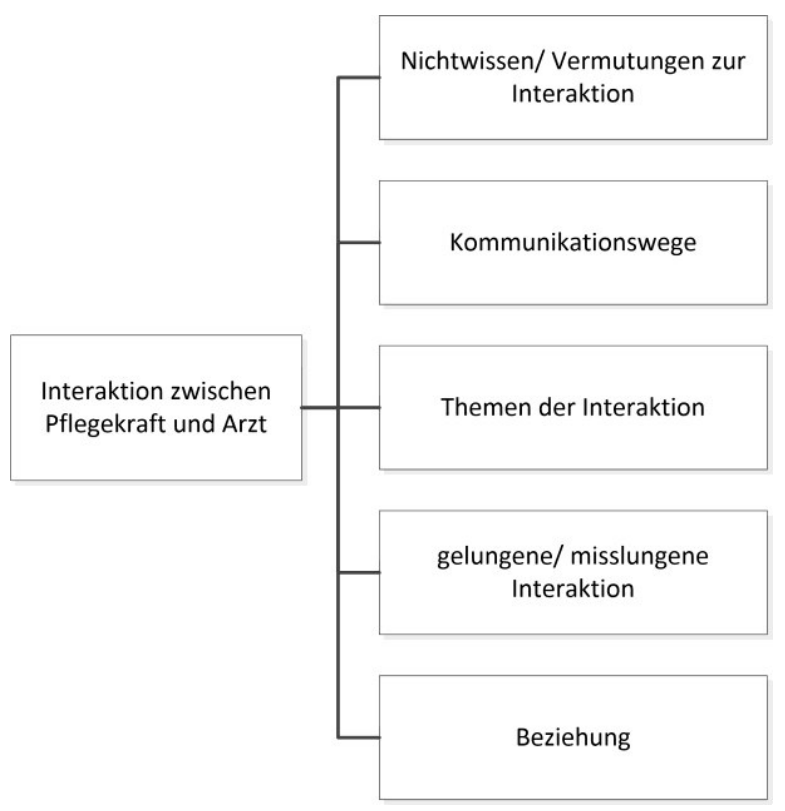

Abb. 9 Kategorie Interaktion zwischen Pflegekraft und Arzt

Interaktion/ Arzt-Pflegekraft/ Nichtwissen/ Vermutungen zur Interaktion

Generell beschreiben viele Bezugspersonen, sie würden von der interprofessionellen Zusammenarbeit nicht allzu viel mitbekommen (CZ2, 94) und könnten sich dazu nicht äußern (AZ1, 168-171; BZ4, 133-134; BZ10, 73-76; CZ6, 40-43), vor allem weil sie häufig nicht dabei wären (BZ2, 119-120; BZ9, 88-89). Andere Bezugspersonen vermuten auch ohne große Einblicke eine gute Zusammenarbeit (BZ2, 115-120; CZ2, 94) oder bewerten den gegenseitigen Umgangs zwischen Arzt und Pflegekraft pauschal positiv.

„[...] da kann ich nix sagen, das ist wahrscheinlich alles in Ordnung“ $(B Z 8,66)$

\section{Interaktion/ Arzt-Pflegekraft/ Kommunikationswege}

Hinsichtlich der Kommunikation zwischen Ärzten und Pflegenden beschreiben einige Angehörige, dass Absprachen häufig im Schwesternzimmer ohne Angehörigen- und Bewohnerbeisein getroffen würden (BZ1, 40; BZ2, 120; BZ4, 143-146). Es gibt jedoch auch Schilderungen, bei denen die Pflegekräfte immer beim direkten Arzt-Patienten-Kontakt anwesend wären (AZ2,66-83; BZ9,93). 
Interaktion/ Arzt-Pflegekraft/ Themen der Interaktion

Aus den Beschreibungen der Bezugspersonen lässt sich herauslesen, dass die Pflegekräfte innerhalb der medizinischen Versorgung mitunter die Rolle einer Art Koordinationsstelle einnehmen. So sind es die Pflegenden, die in der Regel die Entscheidung treffen, ob ein Arzt gerufen wird (AZ1, 188-193; AZ2, 168; BZ2, 44, 81-84; BZ4, 45; BZ9, 93; CZ1, 34; CZ5, 22, 34) und auch sonstigen Bedarf etwa an Medikamenten oder medizinischen Hilfsmitteln an die Praxis weitergeben (AZ3, 112; AZ5, 161-163; AZ6, 158-162).

„[...] dass die Pflegekräfte gemerkt haben, dass es meiner Mutter, dass es irgendwie, irgendwas nicht passt [...]. Dann setzen die sich mit dem Hausarzt in Verbindung oder auch wenn irgendwas anderes ist, wenn die Rezepte braucht, irgendwas [...] Das läuft eigentlich über die Pflegekräfte, über die, über den Hausarzt und das läuft auch gut."

$(C Z 5,28)$

\section{Interaktion/ Arzt-Pflegekraft/ gelungene Interaktion}

Manche Angehörige berichten generell von einem guten Miteinander beziehungsweise Verhältnis zwischen Hausarzt und Pflegekraft, ohne näher darauf einzugehen (AZ2, 221; BZ6, 63-68). Mitunter wird der Eindruck einer guten Zusammenarbeit an reibungslosen Abläufen, etwa Rezeptanforderungen betreffend, festgemacht (AZ6, 158). Eine gute Kommunikation wird hierbei durchaus als etwas Besonders angesehen.

„Also ich glaube nicht, so gut wie es dort läuft. Die Kommunikation zwischen dem [...] Pflegepersonal und den Ärzten, dass das überall so ist."

$(A Z 6,222)$

Andere beschreiben einen guten Kontakt zwischen Arzt und Pflegekraft, da die Ärzte nach stattgefundenem Besuch beim Patienten immer Rücksprache mit den Schwestern halten würden.

„Also es gibt [...] immer Kontakt zur Schwester. [...] die gehen dann extra noch mal zu den Schwestern rein und besprechen es mit denen." (AZ2, 66) 
Für manche Angehörige ergibt sich aus der guten Zusammenarbeit zwischen Arzt und Pflegenden die Vorstellung, selbst weniger in die Versorgung des Bewohners involviert sein zu müssen.

„[...] aber so ärztetechnisch und Pflege. Also das [...] klappt zusammen. Ich habe da noch [...] keinen Notstand oder keinen, ja, Notfall gehabt, dass ich da eingreifen musste, oder was. Das läuft."

$(A Z 3,241)$

Es wird nicht klar, was hier unter „eingreifen“ oder „Notstand“ verstanden wird.

Interaktion/ Arzt-Pflegekraft/ misslungene Interaktion

Fehlende Zeit für das Miteinander wird von den Angehörigen als Hindernis für eine gute Kommunikation zwischen Arzt und Pflegekräften beschrieben (AZ5, 79).

„Wenn ich die Frage [...] zum Beispiel direkt hier stelle im Schwesternzimmer: "Ja was sagt denn der Arzt dazu?" Dann sagen die [Pflegekräfte] so was wie: „Ja was soll der schon dazu sagen, der hat kaum Zeit wenn er hier ist und der hat ja auch noch andere Patienten. " (CZ4, 44)

Ein Mangel an Zeit für eine gute Kommunikation wird sowohl auf Ärzte- als auch auf Pflegeseite beschrieben.

„,ich würde mir meine [...] Aufmerksamkeitsschwelle auch absprechen, wenn jetzt drei Leute auf einmal geklingelt haben, weil sie auf den Schieber müssen. Und wie ich mich dann noch auf diesen Arzt konzentrieren soll, was der mich grade fragt oder was ich dem sagen möchte, finde ich schwierig. Also es ist auch ein Zeitfaktor."

$(\mathrm{CZ4}, 20)$

Als weiteres, die Kommunikation beeinflussendes Element wird angeführt, dass für den Arzt immer nur gewisse Ansprechpartner zur Verfügung stünden, da innerhalb der Pflegeschaft unterschiedliche Qualifikationen aufgrund unterschiedlicher Ausbildung bestünden (BZ2, 120). Außerdem würden sich Ärzte mitunter gar nicht bei den Pflegekräften melden, wenn diese nicht auf Anhieb zu finden seien $(C Z 3,114)$. 
Verbesserungsbedarf sehen Bezugspersonen gelegentlich bei der Verständigung über Medikationen. So würde es bisweilen eine Verunsicherung der Pflegenden nach ärztlichen Hausbesuchen wegen häufiger Medikationswechsel geben (CZ4, 24). Auch gibt es Äußerungen, dass Ärzte und Pflegekräfte sich besser über die Dauer einer Medikamentengabe absprechen müssten. Etwa weil mitunter Medikamente aufgrund schlechter Absprache länger als nötig verabreicht würden (BZ3, 176).

\section{Interaktion/ Arzt-Pflegekraft/ Beziehung}

Die Beziehung zwischen Ärzten und Pflegenden wird von den Angehörigen häufig sehr hierarchisch wahrgenommen und dies bisweilen auch nicht hinterfragt (BZ1, 90; BZ2, 114).

„Und von der Pflege, die machen das [...] was er [Hausarzt] sagt.“ (CZ2, 72)

Andere begründen diese Hierarchie mit der unterschiedlichen Ausbildung beider Berufsgruppen.

„[...] die medizinische Betreuung hat ja [...] der Arzt. Das Pflegepersonal muss das gewissenhaft ausführen, nach ihren Vorlagen, ganz einfach. [...] Und der Hausarzt [...], der hat den Hut auf [...]. Der muss ja entscheiden über die Medikamente, was verträgt sich [...] die [Pflegekräfte] sind zwar auch ein biss[chen] ausgebildet, aber das wissen die ja auch nicht, dass die Medikamente eben sich vertragen und jedes Medikament hat ja seine Nebenwirkung und dass es halt passt."

(CZ7, 96-98)

Den Pflegenden wird etwa nicht viel Einfluss auf Entscheidungen, die medizinische Versorgung betreffend, eingeräumt.

„Also da bemühen sie sich dann schon. Aber sie können sich natürlich gegenüber dem Hausarzt auch nicht unbedingt durchsetzen."

$(\mathrm{AZ6}, 66)$

Es gibt jedoch dazu kritische Meinungen, etwa würde eine Kommunikation nicht 
immer auf Augenhöhe erfolgen und die Pflegekräfte wären den Ärzten "ausgeliefert" (CZ3, 114). Hier scheinen auch eigene Erfahrungen, etwa die Arbeit in einem Pflegeverband, eine Rolle zu spielen.

"[...] die Hierarchien sind ziemlich ausgeprägt und da müssen in Deutschland die Pflegekräfte sich viel mehr professionalisieren, viel mehr sich durchsetzen. Die haben zum Teil ja viel mehr Know-how, kennen die Patienten, Bewohner viel besser als die Ärzte oft. [...] haben aber natürlich nicht die Zuständigkeit und das ist das Problem. “

$(\mathrm{CZ3}, 86)$

Mitunter würde den Pflegekräften das Selbstvertrauen fehlen, um Kritik am ärztlichen Handeln zu äußern.

"[Das] trauen sich erstens nicht die Pflegekräfte, ganz klar, weil da steht ja unser großer Meister Doktor und [...] die haben ja auch sozusagen nicht so sehr viel Einfluss, muss man sagen. Was haben die hier zu sagen? Die haben eigentlich zu funktionieren hier."

$(C Z 4,44)$

Es werden jedoch ebenfalls Situationen geschildert, in denen die Pflegenden bei der Behandlung aktiv mitgestalten und sich etwa für die Anpassung der Medikation einsetzen. Da hier aus der Zusammenarbeit eine Besserung des Gesundheitszustandes des Bewohners resultiert, wird dies von den Angehörigen positiv erlebt (AZ1, 61).

Interaktion/Interaktion zwischen Arzt-Pflegekraft-Bezugsperson

Einige Angehörige machen deutlich, dass sie sich eine bessere Kommunikation zwischen Arzt, Pflegekraft und sich selber wünschen.

„die Kommunikation zwischen Pflege, Arzt und vielleicht auch Angehörigen, da im Dreierverbund könnte da vielleicht biss[chen] noch ausbaufähig sein."

(BZ3, 198-207)

Diese Interaktion zu dritt halten auch andere Bezugspersonen für wünschenswert, schließen darüber hinaus aber noch die Gegenwart des Bewohners mit ein (AZ5, 
257). Eine weitere Bezugsperson bezieht sich dabei explizit auf die Situation am Lebensende der Bewohner, bei der sich im „Team“, was die Angehörigen mit einschließt, über das Vorgehen beraten werden sollte (CZ3, 48-52).

Zusammenfassend lässt sich feststellen, dass Angehörige häufig nicht viel Einblick in die interprofessionelle Zusammenarbeit haben und diese dennoch generell von vielen positiv beschrieben wird. Schwierigkeiten für eine gute Interaktion seien mangelnde Zeit auf beiden Seiten sowie unterschiedliche Kompetenzen der Pflegenden. Auch wird das Verhältnis zwischen Pflegekräften und Ärzten oft sehr hierarchisch erlebt, mit wenig Raum für die Meinung der Pflegenden.

\subsection{Aufgaben der Angehörigen/ Einbezogensein in die medizinische Versorgung}

Hinsichtlich der Frage „Aufgaben der Angehörigen“ hat sich bei der Auswertung der Interviews das Phänomen "Einbezogensein in die medizinische Versorgung“ als zentrales Thema herauskristallisiert. Der Name wurde gewählt, weil Bezugspersonen in unterschiedlichem Ausmaß in die medizinische Versorgung der Bewohner involviert und darüber informiert sind oder sein wollen. Dieses wird im Folgenden anhand des Codierparadigmas nach Strauss und Corbin (Strauss und Corbin 1996) dargestellt, welches die Zusammenhänge und Einflussfaktoren, die mit dem Phänomen in Verbindung stehen bzw. es abbilden, aufzeigt. Wie in Kapitel 4.5.2 beschrieben, ermöglicht dieses Modell die Darstellung von Kontext, ursächlichen und intervenierenden Bedingungen, Handlungsstrategien und Konsequenzen, die hinsichtlich des Phänomens des Einbezogenseins zu finden sind. Im Hinblick auf die Zuordnung zum Codebaum nimmt das Phänomen seinen Ausgang in der Hauptkategorie Bezugsperson. Zusammenhänge und Einflussfaktoren ergaben sich aus den Hauptkategorien Rahmenbedingungen, medizinische Versorgung, Arztbesuch im Pflegeheim, Arzt, Bewohner und Interaktion.

Die Abbildung 10 stellt die Einordnung des Phänomens in das Codierparadigma grafisch dar. 


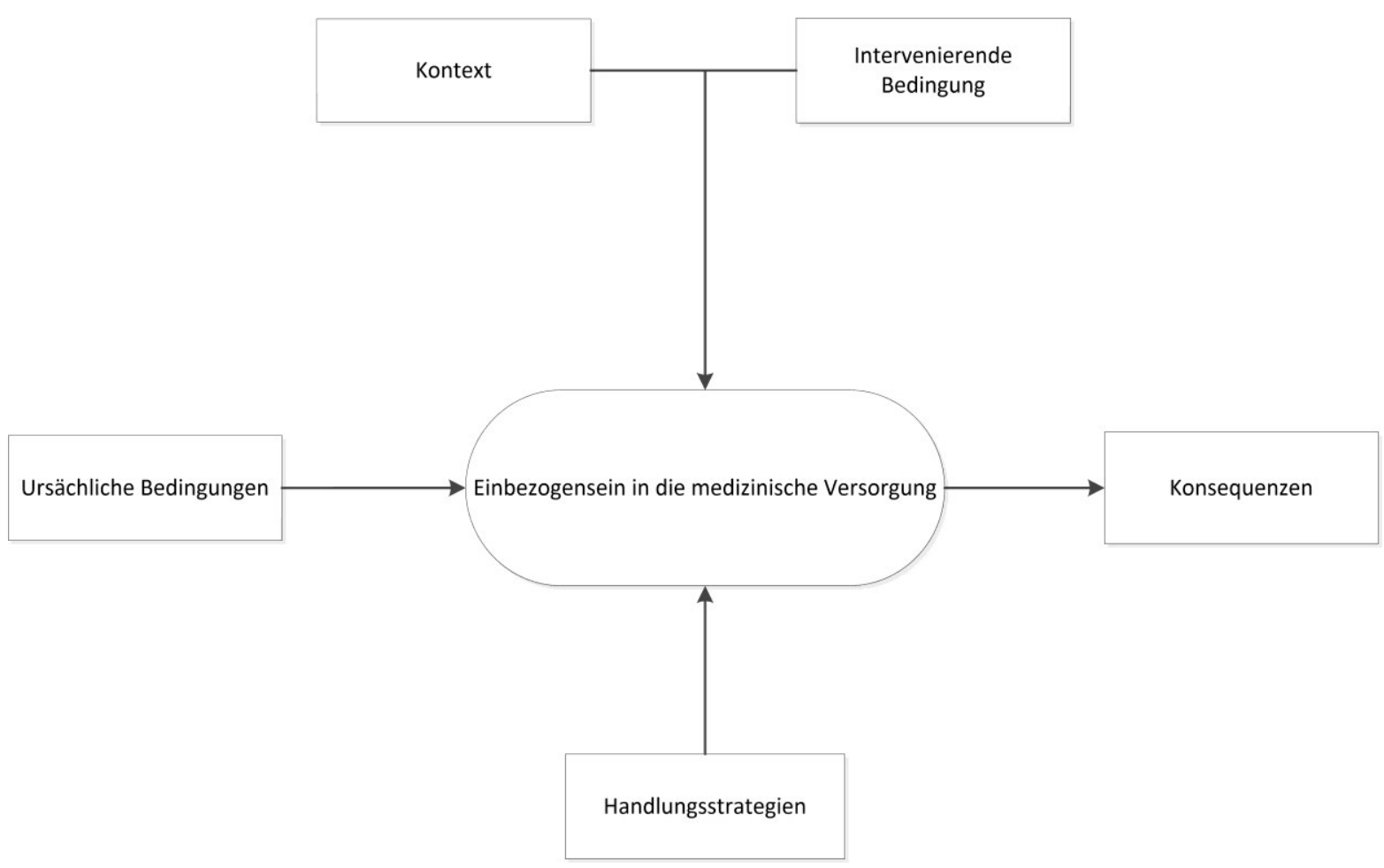

Abb. 10 Codierparadigma nach Strauss und Corbin (Böhm 2012, S.479), die Verwendung der Abbildung erfolgt mit freundlicher Genehmigung des Rowohlt-Verlages, Einbezogensein in die medizinische Versorgung

Im Folgenden werden die einzelnen Abschnitte des Codierparadigmas im Hinblick auf das Phänomen „Einbezogensein in die medizinische Versorgung“ mit Beispielen erläutert und grafisch veranschaulicht.

\section{Kontext}

Das Spektrum des Einbezogenseins der Angehörigen in die medizinische Versorgung der Bewohner, durch von innen ausgeführte, oft selbsternannte Aufgaben ist breit (Abbildung 11). 


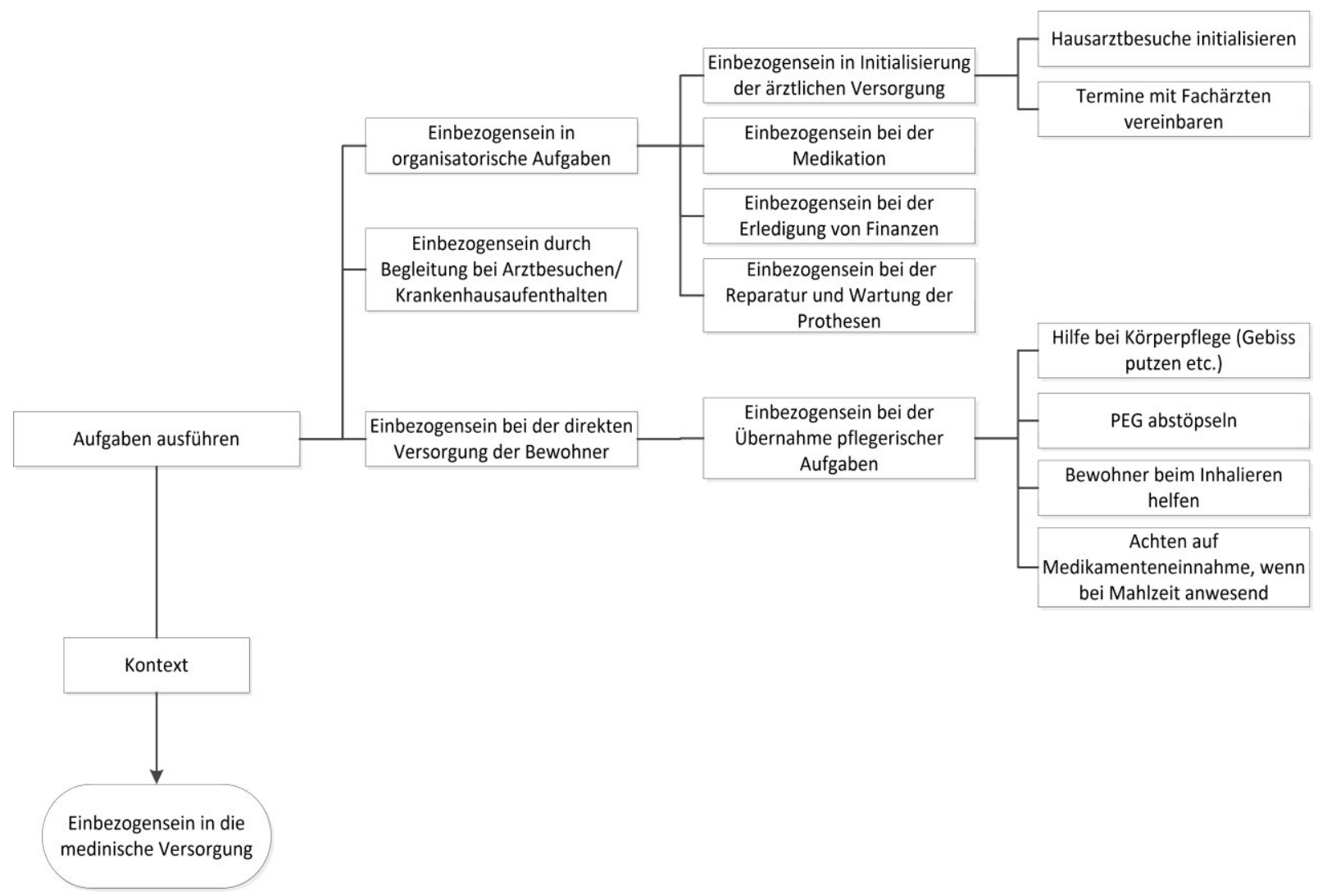

Abb. 11 Kontext, Einbezogensein in die medizinische Versorgung

\section{Einbezogensein in organisatorische Aufgaben}

Einbezogensein äußert sich im Übernehmen vielfältiger organisatorischer Aufgaben durch die Angehörigen bei der medizinischen Versorgung der Bewohner.

Einbezogensein in Initialisierung der ärztlichen Versorgung

Ein Einbezogensein der Angehörigen zeigt sich zudem durch Kümmern um Arztkontakte. Mitunter werden Besuche von Hausärzten initialisiert (BZ8, 18; BZ9, 46, CZ3, 42) und Termine mit spezialisierten Fachärzten ausgemacht (AZ6, 42-44; BZ1, 28; BZ2, 162; BZ9, 61-63, BZ10, 55-62; CZ7, 28).

„Dann gehe ich eben zur Praxis, sage Bescheid: „Mama braucht eine Grippeimpfung, oder möchte eine Grippeimpfung haben, der Doktor möchte bitte in den nächsten Tagen [kommen]."

(AZ3, 97-102) 
Einbezogensein bei der Medikation

Auch hinsichtlich der Medikation lässt sich ein Mitwirken der Angehörigen erkennen. Sie besorgen Rezepte für Medikamente und lösen diese ein (AZ1, 43; $B Z 4,45 ; B Z 10,20 ; C Z 4,36)$ oder beschaffen rezeptfreie Medikamente (AZ5, 165 167).

„Oder auch mal, wenn Sie Medikamente braucht, wenn irgendwas ist, dann gehe ich auch mal und hole das Rezept ab, oder so, bei ihm [Hausarzt] in der Praxis."

$(A 2,170)$

\section{Einbezogensein bei der Erledigung von Finanzen}

Häufig sind Angehörige ebenfalls in sämtliche finanzielle Angelegenheiten der Bewohner einbezogen, indem sie diese regeln (AZ3, 112-114; AZ5, 179-185; AZ6, 16; BZ1, 2; BZ5, 364-372; BZ7, 310; BZ9, 138-141; CZ4, 32), was hinsichtlich der medizinischen Versorgung auch Zuzahlungen zu Arznei-, Hilfs- und Heilmitteln oder Fahrkosten einschließt (AZ1, 116-119; AZ2, 241, 227; AZ4, 132-142, 250; CZ7, 140).

Einbezogensein bei der Reparatur und Wartung der Prothesen

Darüber hinaus übernehmen es die Bezugspersonen mitunter, Hilfsmittel wie Hörgeräte oder Zahnprothesen zur Reparatur oder Wartung zu bringen (BZ2, 164166).

\section{Einbezogensein durch Begleitung bei Arztbesuchen/ Krankenhaus- aufenthalten}

Die Angehörige begleiten die Bewohner von Zeit zu Zeit auch bei ihren Praxisbesuchen (AZ1, 97; AZ3, 229-231; AZ6, 110; BZ1, 28-32; BZ9, 68; CZ7, 28) oder bei Krankenhausaufenthalten (AZ5, 139; BZ10, 82).

„Da haben wir die Augen machen lassen bei der Mutti, weil sie wollte das, sie hatte Grauen Star. [...] Und da war ich natürlich mit in der OP, ich war auch mit im OP-Saal drin, obwohl ich so was nicht so gerne mache, weil da 
bin ich nicht so tapfer, aber ich konnte sie dann an der Hand halten und dann - das ist auch ganz gut gelungen."

(AZ2, 363-365)

\section{Einbezogensein bei der direkten Versorgung der Bewohner}

Die Angehörigen übernehmen nicht nur organisatorische Aufgaben, zum Teil sind sie auch in die unmittelbare Versorgung der Bewohner involviert.

Einbezogensein bei der Übernahme pflegerischer Aufgaben

Die Beteiligung der Angehörigen zeigt sich auch durch direktes Handeln am Bewohner. So werden regelmäßig pflegerische Aufgaben übernommen, beispielsweise bei der Körperpflege der Bewohner (AZ2, 18; BZ5, 204; CZ1, 12; CZ2, 102; CZ6, 19) und mitunter sehr spezielle Tätigkeiten, wie der Umgang mit Ernährungssonden der Bewohner, ausgeübt (AZ5, 123; CZ1, 10).

„Abends putzen die [Pflegekräfte] sowieso nicht die Zähne, habe ich festgestellt. Wenn ich komme, putze ich sie [...]."

$(A Z 5,117)$

\section{Ursächliche Bedingungen}

Für das Einbezogensein der Angehörigen gibt es verschiedene ursächliche Bedingungen, dies sind Aspekte, die das Einbezogensein begründen (Abbildung 12). 


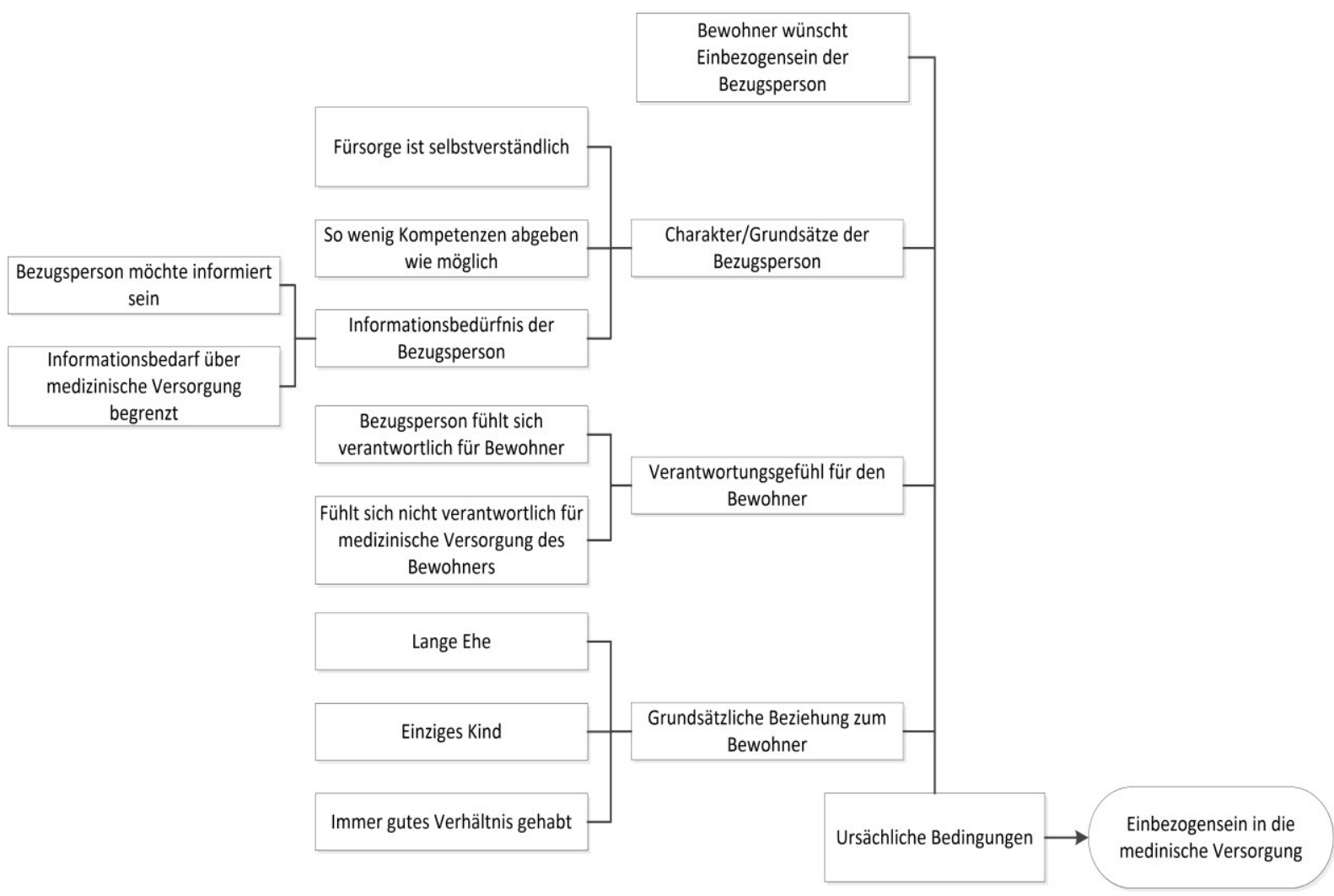

Abb. 12 ursächliche Bedingungen, Einbezogensein in die medizinische Versorgung

\section{Bewohner wünscht Einbezogensein der Bezugsperson}

Ein beeinflussender Faktor auf das Einbezogensein der Bezugsperson ist der Wunsch des Bewohners nach dessen Unterstützung. Bisweilen ist von den Bewohnern die Mitwirkung der Angehörigen etwa durch Übernehmen von Aufgaben wie der Initialisierung von Arztkontakten gewünscht. Ein Grund liegt darin, dass Bewohner möglichst unabhängig von den Pflegenden bleiben möchten, weshalb die Arztbesuche entweder selbstständig organisiert werden oder dies auf die Angehörigen übertragen wird.

„[...] wenn sie [Bewohnerin] merkt, dass das gar nicht funktioniert [Besuche der Hausärztin], dann ruft sie meine Schwester an und meine Schwester organisiert das dann."

$(B Z 10,24)$

\section{Charakter/ Grundsätze der Bezugsperson}

Des Weiteren ist für das Einbezogensein der Bezugspersonen etwa das Ausmaß, in dem sie hinsichtlich der medizinischen Versorgung informiert und involviert sein 
wollen, ursächlich. Für einige ist die Fürsorge für den Bewohner eine Selbstverständlichkeit (AZ1, 134-137; AZ2, 365).

„Wir haben ja auch hinterlegt, dass wir angerufen werden wollen, auch wenn es nachts ist [...]. Weil ich war dann auch mal ganz erstaunt, als mir gesagt wurde [...], dass das explizit vermerkt werden muss, weil Angehörige das nicht wünschen. Ja da war ich ja so sehr geschockt drüber." $(B Z 3,153)$

Zudem möchten einige Angehörige so wenige Kompetenzen wie möglich abgeben, weshalb sie viel in die medizinische Versorgung einbezogen sind (AZ2, 237-239).

„Also ich glaube, dass wir das schlecht können, das abgeben können. Ja,also bei bestimmten Sachen wird auch die Pflege mit einbezogen, aber grad so was es heißt [...] Medikamente oder Physiotherapie und so was, da ist meine Schwester schon auch noch mit [...] dabei [...]. ich glaube nicht, dass meine Schwester das ganz loslassen würde."

(BZ10, 130-134)

Das Informationsbedürfnis der Bezugspersonen ist unterschiedlich stark ausgeprägt. So wollen manche nicht über alles, was den Bewohner betrifft auch hinsichtlich der medizinischen Versorgung informiert werden (BZ5, 373-377).

„Ich weiß auch nicht, wie das mit den Tabletten geht. Ob sie da immer aufpassen, dass sie sie nimmt, oder so. Das weiß ich natürlich alles nicht. Sie kriegt sie hingestellt zu den Mahlzeiten und keine Ahnung, wie das funktioniert. Ich bin ja dann nicht da. Ich habe mir jetzt vorgenommen, ich muss das nicht alles wissen. Das regt mich einfach viel zu doll auf [...], wenn ich das immer alles mitkriege."

$(A Z 1,219)$

Andere hingegen möchten gerne über alle den Bewohner betreffenden Vorgänge informiert sein (AZ2, 221-227). 


\section{Verantwortungsgefühl für den Bewohner}

In diesem Zusammenhang wird noch eine weitere ursächliche Bedingung für das Einbezogensein, ein Verantwortungsgefühl für die Bewohner, von Angehörigen deutlich.

„Also [...] leider bin ich ein Typ, der immer denkt, er ist für Vieles verantwortlich. Gut, das ist ein bisschen größenwahnsinnig, aber ich weiß schon immer gerne über alles Bescheid, was Mutti betrifft. [...] wie viel Tabletten da drin sind und wie lange die dann gereicht haben, das sehe ich schon, dass ich auch ein gutes Gefühl habe und dass ich weiß, was sie so einnimmt und so. Das ist mir schon ein bisschen wichtig, ein bisschen darüber Bescheid zu wissen."

(AZ2, 221-227)

Andere Bezugspersonen geben mit dem Eintritt ihres pflegebedürftigen Angehörigen Verantwortung an das Heim ab und fühlen sich für die Versorgung des Bewohners weniger verantwortlich.

„ICh bin halt immer mit ihr [Bewohnerin] zum Arzt gegangen und wusst[e] genau, was Sache ist. Und sie erzählt mir ja auch ständig von Rückenproblemen und da kann ich das aber besser einschätzen, ob ich jetzt denk, dass sie da schon wieder eine neue Tablette braucht oder nicht. Das krieg[e] ich jetzt halt so nicht mehr mit."

$(B Z 1,10)$

\section{Grundsätzliche Beziehung zum Bewohner}

Dieses Verantwortungsgefühl zeigt sich auch bei anderen Bezugspersonen und ergibt sich mitunter aus der Beziehung zum pflegebedürftigen Angehörigen, etwa aufgrund eines langjährigen guten Verhältnisses, einer langen Ehe oder auch durch den Umstand, einziges Kind zu sein.

„Ich weiß nicht [...] wenn ich nicht da wäre, wie das laufen würde. Ich fühle mich halt auch verantwortlich, weil ich ihre Tochter bin und das einzige Kind [...]."

(AZ2, 90)

Folglich ist eine weitere ursächliche Bedingung für die Teilhabe der Bezugspersonen die zu Grunde liegende Beziehung zum Bewohner. 


\section{Intervenierende Bedingungen}

Bezogen auf das Phänomen Einbezogensein in die medizinische Versorgung ergeben sich viele intervenierende Bedingungen, dies bedeutet beeinflussende Faktoren (Abbildung 13).

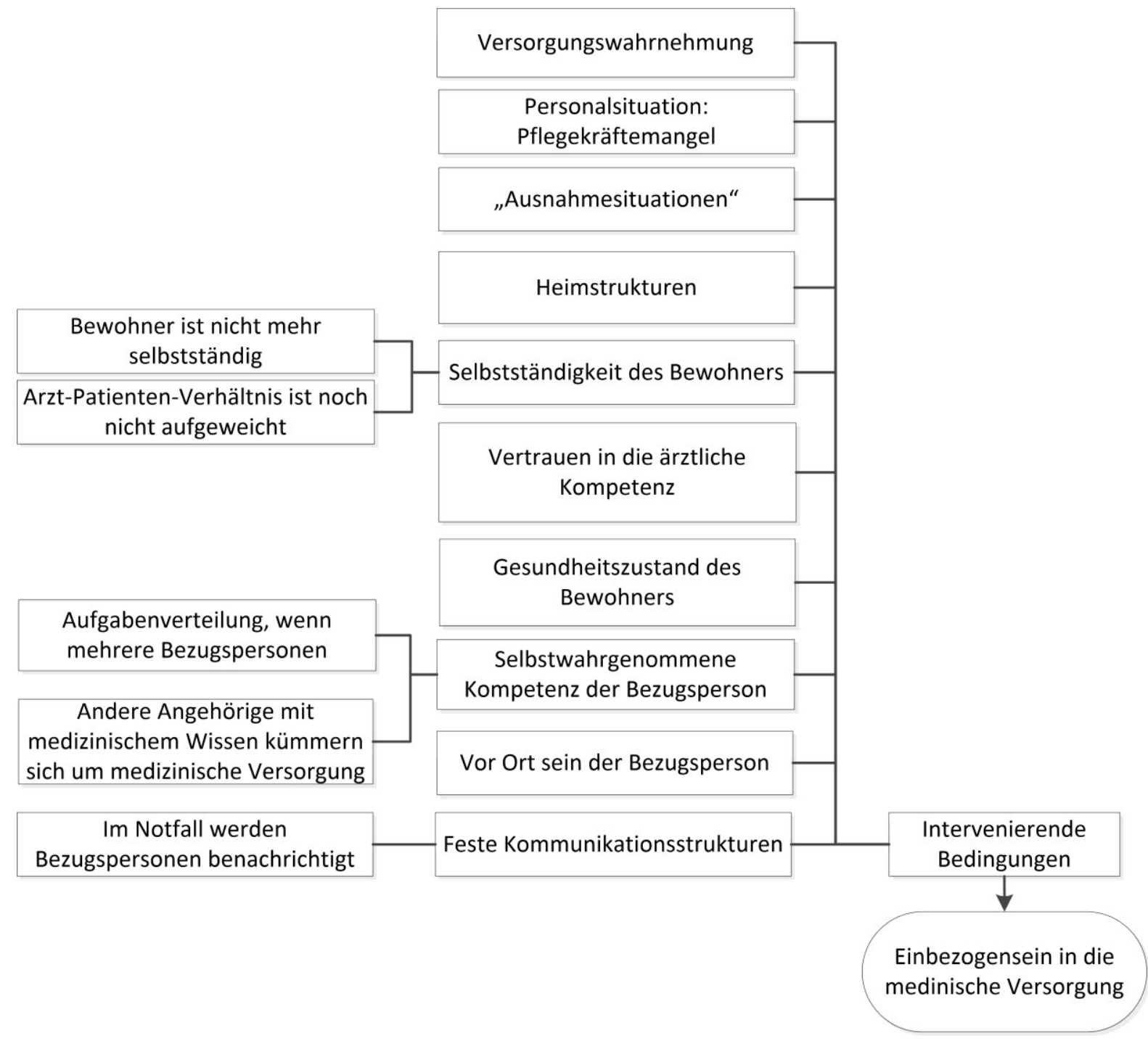

Abb. 13 intervenierende Bedingungen, Einbezogensein in die medizinische Versorgung

\section{Versorgungswahrnehmung}

Die medizinische Versorgungswahrnehmung führt zu mehr oder weniger Einbezogensein der Angehörigen. 
So besorgen Bezugspersonen bei einer subjektiv nicht funktionierenden Versorgung etwa Rezepte selbstständig (AZ1, 43) oder initialisieren Hausarztbesuche, um eine Versorgung zu beschleunigen $(C Z 3,42)$.

„Wo das mit dem Rezept nicht geklappt hat, mit der Krankengymnastik, habe ich sie [Hausärztin] auch direkt einmal angerufen und hab gesagt: „Er [Bewohner] hat noch kein Folgerezept. “"

$(\mathrm{BZ4}, 65)$

Andererseits halten sich Angehörige eher zurück, wenn die medizinische Versorgung ihrer Meinung nach funktioniert, was unter anderem an der Medikamentenverordnung oder der Zufriedenheit der Bewohner selbst festgemacht wird (BZ5, 377; CZ5, 58).

\section{Personalsituation: Pflegekräftemangel}

Ein größeres Einbezogensein von Angehörigen kann auch aufgrund der subjektiven angespannten Personalsituation entstehen, um die Pflegekräfte zu entlasten. Einbezogensein zeigt sich hier darin, dass Angehörige sowohl pflegerische Aufgaben (BZ6, 16; CZ6, 6), als auch Aufgaben hinsichtlich der medizinischen Versorgung, etwa die Besorgung von Rezepten, übernehmen.

„Oder auch mal, wenn sie [Bewohnerin] Medikamente braucht, wenn irgendwas ist, dann gehe ich auch mal und hole das Rezept ab, oder so, bei ihm [Hausarzt] [...] in der Praxis. Oder früher mit den 10€, habe ich auch noch das Kärtchen hingebracht und habe die $10 €$ bezahlt bei ihm. [...] Und dann kam er halt irgendwann in der Woche. Also das habe ich schon abgewickelt, dass sie [Pflegekräfte] ein bisschen hier auch Luft haben. Weil die Schwestern arbeiten schon viel und manchmal denke ich, wenn einer ausfällt und einer krank ist, ist es schon übel, manchmal."

$(A Z 2,170)$

\section{„Ausnahmesituationen“}

Auch Ausnahmesituationen erfordern ein kurzfristiges Einbezogensein von Angehörigen. Wenn Arzneimittel umgehend besorgt werden sollen, übernehmen die Angehörigen diese Aufgabe und entlasten damit die Pflegekräfte (BZ4, 45).

"Ich bin auch schon selbst in die Praxis gefahren, um da ein Rezept abzuholen, was dann hier sehr [...] plötzlich, sehr dringend und sehr notwendig war, [...] laut den Schwestern." 


\section{Heimstrukturen}

Darüber hinaus führen die in den Heimen gegebenen Strukturen, etwa die Versorgung mit spezialisierten Fachärzten, zu mehr oder weniger Beteiligung/ Einbezogensein der Bezugspersonen. So sind je nachdem, ob spezielle Kooperationen des Heims mit bestimmten Fachärzten bestehen, die Angehörigen nicht mehr an der Initialisierung solcher Besuche beteiligt (AZ3, 20-24; BZ4, 153156) oder übernehmen die Organisation, weil derartige Termine nicht vom Heim arrangiert werden $(\mathrm{BZ1}, 28)$.

"[...] und also Arztbesuch so was müssen wir regeln. [...] da haben sie [Pflegekräfte] mich gleich aufgerufen, sie [Bewohnerin] muss dringend zum HNO."

(BZ9, 61-63)

\section{Selbstständigkeit des Bewohners}

Werden die Bewohner noch als selbstständig angesehen, führt dies zu einem geringeren Einbezogensein. Hier verspüren die Angehörigen wenig Bedürfnis, sich in die ärztliche Versorgung, etwa durch eigene Gespräche mit dem Arzt, einzumischen und die Teilhabe an der Versorgung des Bewohners ist geringer $(B Z 1,10)$.

\section{Vertrauen in die ärztliche Kompetenz}

Ein Vertrauen in das ärztliche Handeln führt mitunter zu weniger Einbezogensein der Angehörigen.

„ja gut [...] zu Medikamenten und das kann ich also nix sagen, weil [...] da hab ich Vertrauen [...] zum Arzt und da hab ich mich auch nicht weiter [...] schlaugemacht. Also wenn mir was aufgefallen wäre dann ja, aber so [...] kann ich nix sagen."

$(B Z 8,6)$

\section{Gesundheitszustand des Bewohners}

Auch ein subjektiv geringes Kranksein des Bewohners führt zu weniger Beteiligung/ Einbezogensein der Angehörigen (BZ8, 6). 
[Hausarzt] sich auch melden würde, aber das, was jetzt als Routine war, das haben wir dann erst hinterher erfahren entweder [...] von den Pflegekräften."

(BZ3, 57-59)

\section{Selbstwahrgenommene Kompetenz der Bezugsperson}

Auch die selbstwahrgenommene Kompetenz kann das Einbezogensein beeinflussen. Gibt es mehrere Bezugspersonen, werden Aufgaben auch zwischen verschiedenen Bezugspersonen je nach Kompetenz aufgeteilt (BZ10, 82). Angehörige mit medizinischem Wissen achten dabei verstärkt auf die medizinische Versorgung der Bewohner.

„meine Schwester lebt noch [...], die ist Krankenschwester und wenn die da ist, [...] dann schaut sie natürlich mehr nach den Medikamenten. Die weiß auch, was das ist und die kann auch mit den Pflegern da fachmännisch reden."

$(B Z 8,6)$

\section{Vor Ort sein der Bezugsperson}

Um einbezogen zu sein, müssen die Bezugspersonen vor Ort sein. So wäre es beispielsweise bei durch die Pflege mitgeteilter nötiger Krankenhauseinweisung des Bewohners noch möglich, zeitnah ins Heim zu fahren, um die Krankenhauswahl zu beeinflussen (AZ6, 64-66). Auch erlaubt das vor Ort sein der Bezugsperson die eigenständig veranlasste Verständigung des Hausarztes bei akuten Gesundheitsbeschwerden.

„Also, wenn ich da wäre, würde ich wahrscheinlich handeln. Aber [...] wenn ich morgens da war und das am Mittag passieren tät, [...] würde die Schwester natürlich, denke ich, handeln. “

(AZ2, 168)

\section{Feste Kommunikationsstrukturen}

Teilhabe der Angehörigen zeigt sich auch in Notfallsituationen, wenn Angehörige benachrichtigt werden möchten. Hierfür gibt es häufig feste Absprachen mit dem Pflegeheim (AZ3, 50-56; AZ4, 22; BZ1, 44-46; BZ3, 51-53; BZ5, 111-113). In diesem Zusammenhang spielen auch bereits gemachte Erfahrungen eine Rolle 
oder auch der Wunsch nach Einbezug in Entscheidungen über eventuelle Krankenhauseinweisungen.

„Ich möchte das nicht, dass das über meinen Kopf hinweg, irgendwas passiert."

(AZ1, 154-155)

„Und dann habe ich ihr [Notärztin] auch erklärt, warum ich da so strikt dagegen [Krankenhauseinweisung] war. Ich habe nämlich meine erste Stelle beim Landeskrankenhaus, das ist vergleichbar mit diesem hier, und da habe ich ja gesehen, was da los ist, nicht? Und das wollte, nein, also das wollte ich nicht. [...] da hat sie zu mir gesagt: „Können Sie das denn verantworten?" „Ja“, sage ich, „mehr als hier schlafen kann ich ja nicht." Ich habe hier vier Nächte campiert."

$(A Z 1,131)$

\section{Handlungsstrategien}

Die Handlungsstrategien der Angehörigen ergeben ein unterschiedliches Maß an Einbezogensein in die medizinische Versorgung. Es werden viele Handlungen für den Bewohner aktiv ausgeführt, darüber hinaus wird bisweilen versucht Einfluss zu nehmen oder Kontrolle auszuüben, auf der anderen Seite aber auch Verantwortung an das Pflegeheim abgegeben (Abbildung 14).

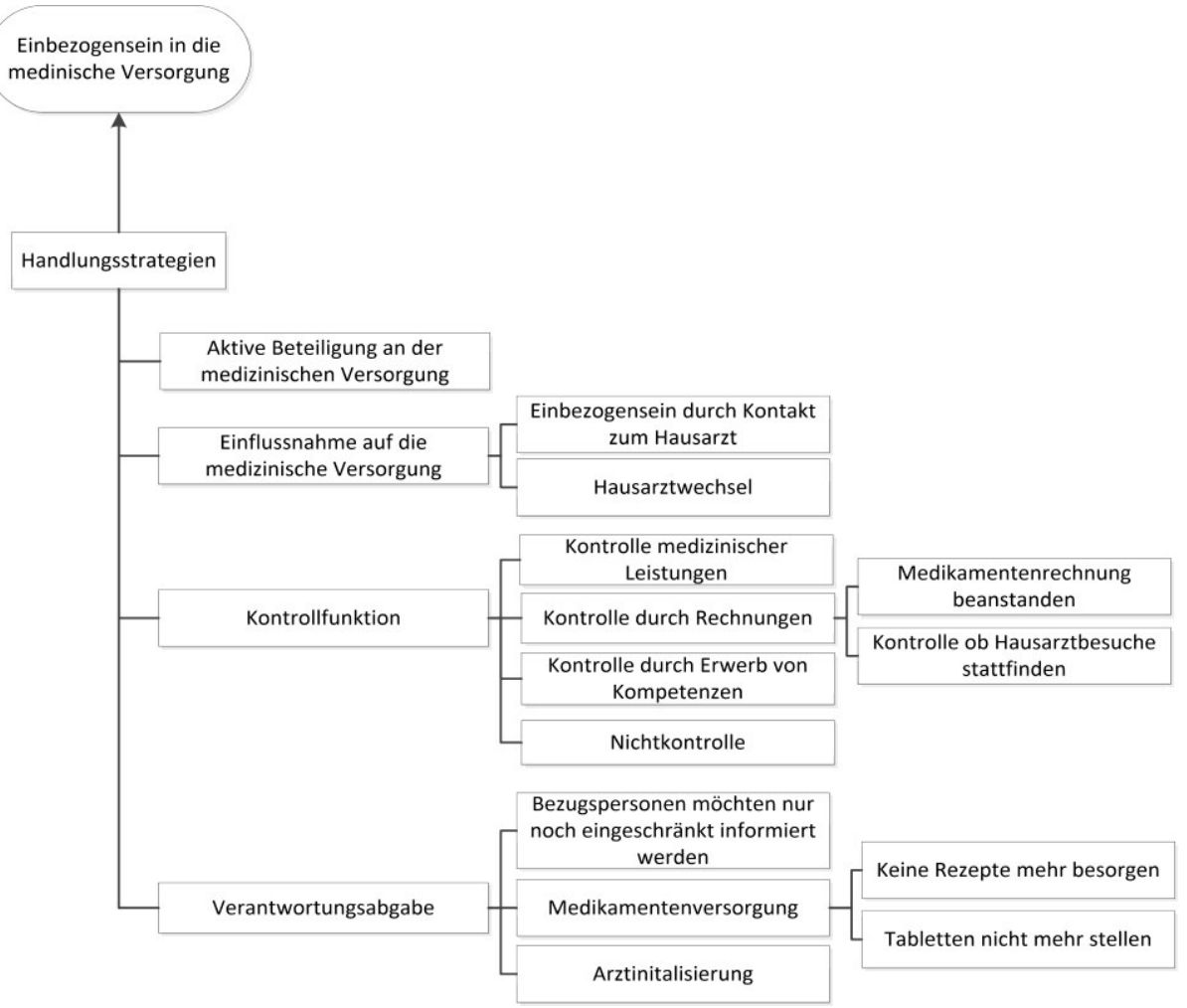

Abb. 14 Handlungsstrategien, Einbezogensein in die medizinische Versorgung 


\section{Aktive Beteiligung an der medizinischen Versorgung}

Angehörige führen wie bereits in diesem Kapitel unter Kontext aufgeführt aktiv Aufgaben in der medizinischen Versorgung aus. Dies geschieht sowohl hinsichtlich organisatorischer Aufgaben, etwa der Initialisierung von Arztkontakten oder der Beschaffung von Medikamentenrezepten, als auch bei der direkten pflegerischen Versorgung, wie der Hilfe bei der Körperpflege der Heimbewohner.

\section{Einflussnahme auf medizinische Versorgung}

Angehörige berichten von Situationen, in denen sie in unterschiedlicher Art und Weise aktiv versuchen, auf die medizinische Versorgung Einfluss zu nehmen.

Einbezogensein durch Kontakt zum Hausarzt

Das Einbezogensein der Angehörigen äußert sich etwa in der Häufigkeit und der Qualität ihrer Kontakte zu den Hausärzten der Bewohner (siehe Kapitel 5.2.3 Interaktion zwischen Bezugsperson und Hausarzt). So ist eine Handlungsstrategie die gezielte Kontaktsuche bei bestehendem Gesprächsbedarf (AZ5, 97-103; BZ7, 62-66; CZ4, 36).

Angehörige suchen den Kontakt etwa, um sich über den Gesundheitszustand und die Pflegebedürftigkeit des Bewohners auszusprechen (AZ5, 103). In anderen Fällen wurden Beobachtungen zum Gesundheitszustand des Bewohners gemacht, die Angehörige gerne darlegen möchten (BZ7, 62-70) oder es besteht der Wunsch, Einfluss auf die Behandlung zu nehmen (CZ4, 12).

"[...] wenn es um Krankengymnastik geht zum Beispiel. Da hat [es] letzte[s] Mal geheißen, es gibt kein Rezept mehr. Und dann spreche ich auch mal mit ihr [Hausärztin] und dann sagt sie entweder: es ist ein Folgerezept möglich oder es ist keines mehr möglich."

$(B Z 4,59)$

„ICh sag mal, es kommt immer drauf an, ob das erforderlich ist. Solange nichts Besonderes anliegt und die Verordnung von den Medikamenten, von den [...] Therapien, das alles läuft und dann ist das für mich so in Ordnung. Wenn dann irgendwas anliegt, dann denke ich, wird das auch kein Problem sein, dass wir [Hausarzt und Angehörige] uns treffen, dass ich dabei bin. Also da [...] bin ich mir ziemlich sicher."

$(\mathrm{CZ} 5,58)$ 
Hausarztwechsel

Ein Einbezogensein der Angehörigen liegt bisweilen auch bei Hausarztwechseln vor. So sind die Angehörigen mitunter an der Entscheidung, den Hausarzt zu wechseln, beteiligt, wenn eine Unzufriedenheit mit der ärztlichen Versorgung vorliegt. Dies kann durch Probleme mit der medikamentösen Versorgung, oder auch mit nicht stattfindenden Kontrolluntersuchungen zusammenhängen.

„[...] war bekannt, dass sie [Bewohnerin] Zucker hat. Seit Januar, seitdem sie hier ist, hat die [Hausärztin] nicht einmal Zucker kontrolliert, auch nicht die Nierenwerte kontrolliert, die nicht in Ordnung waren. "Ja, wir überprüfen das." Sie soll ein Tagesprofil machen. Und das Tagesprofil streckte sich fast über vierzehn Tage. Da waren Werte von 325 dabei. Und 80-120 ist normal. Und dann heißt das: „Ja, und die vielen Tabletten, die sie nimmt, und das Alter. Das ist gar nicht so schlimm." Und da haben wir gesagt: „Nein, also nun ist Schluss!"

$(A Z 1,65)$

Durch Angehörige initiierte Hausarztwechsel können auch stattfinden, weil eine Unzufriedenheit mit der ärztlichen Versorgung, etwa einer unzuverlässigen Besuchsfrequenz des Arztes, vorliegt und die medizinische Versorgung des Bewohners gefährdet erscheint $(\mathrm{CZ1}, 50)$.

\section{Kontrollfunktion}

Das Einbezogensein der Angehörigen in die medizinische Versorgung äußert sich zuweilen auch in der Ausübung einer gewissen Kontrollfunktion. So schätzen sich einige Angehörige als „Kontrollinstanz“ ein und geben zu bedenken, ob die pflegerische Versorgung des pflegebedürftigen Angehörigen ohne ihre Präsenz schlechter wäre (CZ4, 2; CZ6, 17). Diese Zweifel werden auch in Bezug auf die medizinische Versorgung formuliert. So wird die gute Versorgung des Heimbewohners mitunter auf die Angehörigenpräsenz zurückgeführt und Situationen geschildert, in denen Pflegeheimbewohnern, vermeintlich ohne Angehörige, nicht ausreichend medizinisch versorgt worden wären.

„[da]durch, [...] dass wir halt sehr oft präsent sind, ist es okay. [...] Wir haben auch schon von Leute[n] andere Sachen gehört [...], wo eine Patientin mal zum Arzt gemusst hätte, wo halt nix passiert ist. Ja da ist kein Arzt gekommen, sie ist auch nicht zum Arzt gefahren worden. [...] Wir haben 
jetzt nur im Moment die Situation, dass wir halt sehr viel präsent sind, aber wie es ist, wenn wir nicht so oft präsent wären."

$(B Z 4,271)$

Kontrolle medizinischer Leistungen

Andere Angehörige kontrollieren die Versorgung des Bewohners durch Nachfragen, etwa ob Physiotherapiestunden stattgefunden haben.

„[...] ich passe auch so ein bisschen auf. Ich frage dann immer: „Mutti, [...] war sie [Physiotherapeutin] da?"

$(A Z 2,237)$

Kontrolle durch Rechnungen

Da die Angehörigen häufig die finanziellen Angelegenheiten der Bewohner erledigen, ergibt sich für sie die Möglichkeit anhand von Rechnungen, erbrachte ärztliche Leistungen (BZ5, 344-358) und Medikamentenverschreibungen nachzuvollziehen (BZ9, 142). Mitunter werden Rechnungen für Medikamente dann auch beanstandet.

„Wenn man also nachguckt [...] in denverordnungs- und verschreibungspflichtigen Medikamenten, wo ich mich sehr genau, gerade in dem Bereich Schmerzen, Drogen auskenne, sieht man [...], dass die nicht auf Privatrezept verordnet werden müssen. Das heißt, nach den Anlagen der Gesetzgebung nur verordnungsbedingt sind. Und [...] das bedeutet auch, dass ich als Angehörige, Kosten [...] sind auch immer ein Thema, immer [...] diese Rechnungen prüfen muss und da Unterschiede finde - monatliche Rechnung 170 Euro."

$(C Z 4,12)$

Zudem wird bisweilen geprüft, ob abgerechnete Hausarztbesuche stattgefunden haben.

„Das ist [...] [ei]ne Praxis mit vier Ärzten und naja meine Frau hatte einen davon, ich hatte einen anderen und sie wird regelmäßig von [...] [der]Praxis mit Besuchen betreut. Soviel ich weiß einmal die Woche. Ich weiß nur noch nicht, ob sie diese Woche schon da waren. Also wenn sie das vergessen mir zu sagen, dann frag ich nach [...]. Na ich seh[e] es ja von der Apotheke, die Rechnung, die ich dann kriege also." 
Darüber hinaus wird von den Bezugspersonen bisweilen angestrebt, einen Überblick über die Medikation der Bewohner zu behalten. So wird das Auskommen mit einer Arzneimittelpackung mit den Rechnungen abgeglichen, um informiert zu sein, welche Medikamente vom pflegebedürftigen Angehörigen eingenommen werden (AZ2, 221-227).

Kontrolle durch Erwerb von Kompetenzen

Andere Angehörige erwerben gezielt Wissen, um Aufgaben übernehmen zu können und so ihre Teilhabe zu verstärken. So werden beispielsweise Bedienungsanleitungen von Ernährungssonden gelesen, um selbst Handhabungen durchführen zu können und die Versorgung durch die Pflege zu kontrollieren.

„Das steht ja drin in der Beschreibung. [...] So ein dickes Heft. Die Anwendungen und so. Mit denen kenne ich mich bald besser aus als die [Pflegekräfte] da. [...] manche setzen sie entweder in den Aufenthaltsraum mit dem [...] Schlauch dran, mit dem PEG Schlauch da. Oder auf dem Zimmer, manche. [...] Und dann klemme ich dann ab, spüle das durch, weiß ich, darf nicht verstopfen, nicht? Und [...] schalte dann natürlich aus das Ding und [...] das Anklemmen nachher, sage ich, sollen die mal machen, die bringen sie ja sowieso ins Bett. Soviel Zeit braucht sie da nicht mit dem Essen."

(AZ5, 120-123)

Nichtkontrolle

Wenig Beteiligung der Angehörigen liegt vor, wenn diese bewusst nicht in die medizinische Versorgung einbezogen sein wollen. Bisweilen sind sie etwa nicht gewillt, beispielsweise die Medikamentenversorgung zu kontrollieren, da eine funktionierende Versorgung voraussetzt wird.

„[...] ich kann das nicht weiter überprüfen. Ich sehe es abends, dass sie [Bewohnerin] [...] so ein kleines Gefäß mit Tabletten bekommt. Das wird wahrscheinlich auch morgens sein, aber ich meine, sonst könnte ich sie gleich mit nach Hause nehmen, wenn das nicht läuft. Nein, ich bin sowieso der Meinung, man zahlt sehr viel hierfür. Und wenn das nicht läuft, ist das eine Schweinerei. Davon gehe ich dann auch aus. [...] Und ich will es nicht kontrollieren."

(AZ4, 109-122) 


\section{Verantwortungsabgabe}

Das Einbezogensein wird verringert, wenn Angehörige bezüglich der medizinischen Versorgung zu ihrer Entlastung Verantwortung an das Heim abgeben und nur noch eingeschränkt informiert werden wollen.

Bz2: „Wenn man das sieht, wenn man alles wüsste, das wäre vielleicht zu viel, wenn die uns immer Bescheid gegeben: „Die Mutter hat Husten, die Mutter hat das. "Das wird dann vielleicht zu viel."

Bz1: „Nee, ich mache mir zu viel Sorgen. Mir ist es jetzt eigentlich so recht, wenn nichts Gravierendes ist. "

Bz2: „Mmh, dass die das machen ja.“

Bz1: „Dass die das machen ja. Mir gehen dann so viele Dinge im Kopf rum, dann kann ich nicht einschlafen. So [...] Kleinigkeiten will ich gar nicht wissen. Wir sagen immer, [...] wenn wir sehen, der Mutter geht es gut, wir gehen zufrieden raus und [...] dann haken wir da eigentlich nicht nach, wenn wir sehen, sie fühlt sich wohl."

(BZ5, 373-377)

Andere Formen der Verantwortungsabgabe an das Pflegeheim finden, auch wenn derartige Aufgaben vor Heimeinzug übernommen wurden, etwa hinsichtlich der Medikamentenversorgung (AZ2, 323; AZ3, 112; AZ4, 102; BZ5, 44; CZ7, 96) oder der Initialisierung von Arztbesuchen statt.

„Oder [...] sie muss zum Arzt, wobei ich das früher auch viel selber gemacht habe, dass ich mit ihr zum Arzt gefahren bin, aber das geht jetzt alles nicht mehr. Und [...] von daher [...] müssen die [Pflegekräfte] das dann veranlassen oder es kommt jemand ins Haus."

$(A Z 6,18)$

\section{Konsequenzen}

Für das Einbezogensein resultieren durch die Beteiligung der Bezugspersonen verschiedene Konsequenzen (Abbildung 15). 


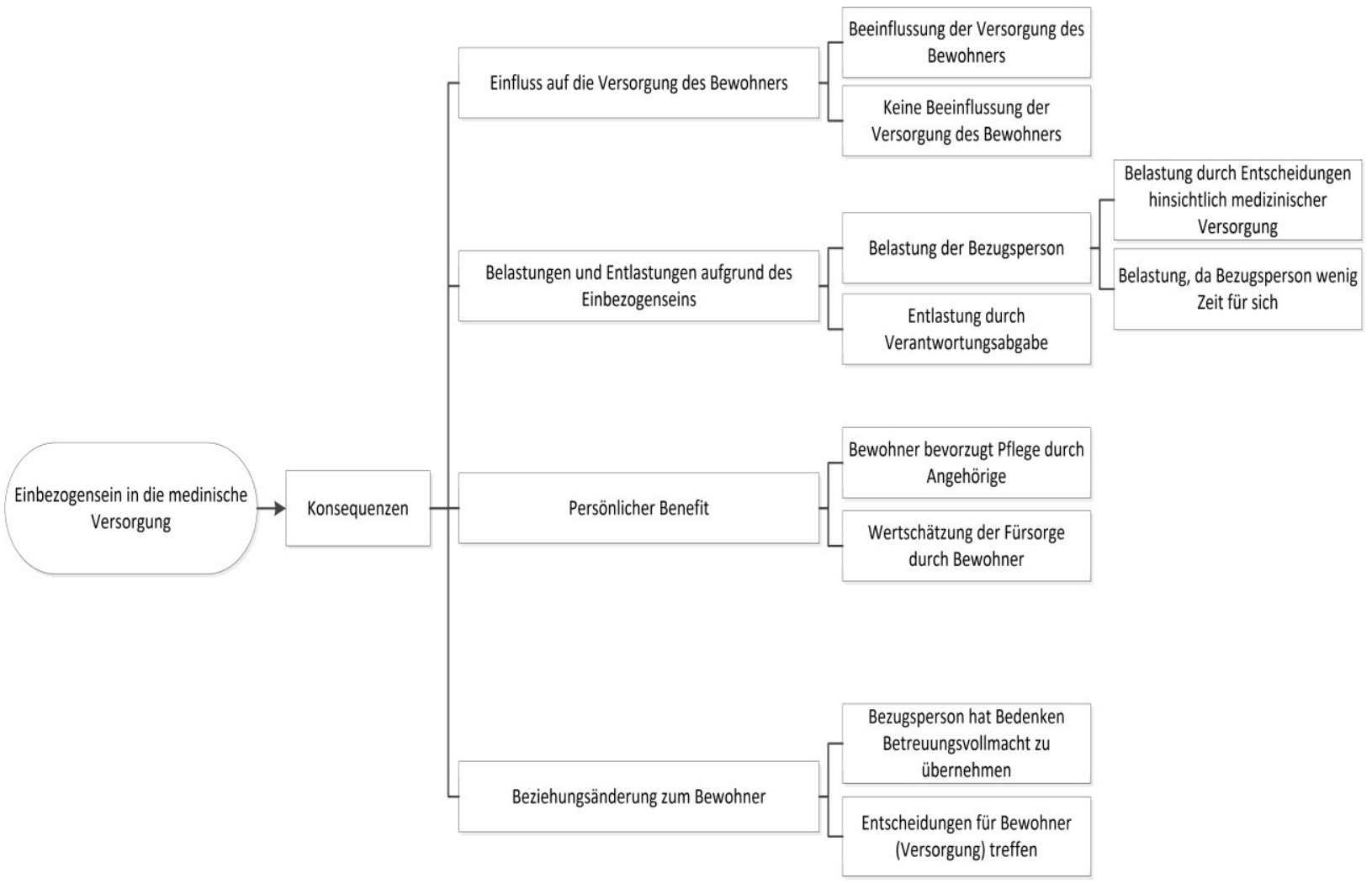

Abb. 15 Konsequenzen, Einbezogensein in die medizinische Versorgung

\section{Einfluss auf die Versorgung des Bewohners}

Als Konsequenzen der Handlungen und des Einbezogenseins der Bezugspersonen wird die Versorgung des Bewohners zum Teil beeinflusst.

Beeinflussung der Versorgung des Bewohners

Durch die Beteiligung der Angehörigen erfolgen bisweilen etwa Krankenhauseinweisungen (AZ2, 86). Andererseits werden ebendiese auch durch Gespräche mit dem Arzt verhindert (AZ1, 127-131).

„Na ja, die [Bewohnerin] ist auf mein Betreiben hin in die Uni gekommen, weil sie Schmerzen hatte. Hüftgelenkschmerzen und die schrie dann vor Schmerzen. Auch bei Bewegung und Drehen [...] und das war nicht nur einmal, sondern das war eine Woche und zwei Wochen sogar. So und das sagte ich dem Pfleger, ich sagte: „Das ist doch unmöglich. [...] 
Da muss man doch das nach sehen oder was machen!" „Ja.", sagt er, "Machen wir."

(AZ5, 137-139)

Keine Beeinflussung der Versorgung des Bewohners

An anderer Stelle wird deutlich, dass der Einfluss der Angehörigen durchaus begrenzt ist. So versuchen Bezugspersonen bisweilen zwar durch Gespräche mit dem Hausarzt oder der Pflegedienstleitung, Änderungen der Behandlung herbeizuführen, mitunter jedoch vergeblich.

"[...] ich versuche immer drauf hinzuwirken, dass meine Mutter nicht so hoch dosiert ist. Habe ich hier schon Kriege geführt, kann ich sagen und [...] sehe immer wieder, dass immer wieder die Höchstmedikation aufgeschrieben wird $[\ldots]^{\prime \prime}$

$(\mathrm{CZ4}, 12)$

\section{Belastungen und Entlastungen aufgrund des Einbezogenseins}

Konsequenzen des Einbezogenseins in die medizinische Versorgung sind zudem Belastungen für die Bezugspersonen oder die Entlastung durch Verantwortungsabgabe an die Pflegeeinrichtung.

\section{Belastung der Bezugsperson}

Durch die Betreuung der pflegebedürftigen Angehörigen, etwa auch Begleitung bei Arztbesuchen und Krankenhausaufenthalten, bleibt unter anderem wenig Zeit für die eigene Person.

„Aber [...] also es ist schon, es ist für Mutti bestimmt ganz, ganz schlimm, aber es ist für uns auch eine Belastung. [...] Es ist nicht nur alles Freude, was man auch viel mit Freude macht. Aber es ist schon eine Belastung, ja."

(AZ2, 383)

Belastungen entstehen auch, wenn Bezugspersonen wesentliche Entscheidungen in medizinischen Fragen stellvertretend für die Bewohner treffen müssen. Hier kommt es mitunter im Nachhinein zu Gewissenskonflikten und Vorwürfen.

„Mit künstlicher Ernährung haben wir damals zugestimmt. Auch in [der] Patientenverfügung nachher zum Schluss noch. [...] dass Sie - naja künstlich ernährt wird, weil wir dachten, die [Bewohnerin] macht vielleicht nicht mehr allzu lange und machst dir Vorwürfe, nicht, weil die Ärzte an der Uni: „Ja! Und wir wollen sie ja nicht verhungern lassen und das bessert sich 
ja wieder und wird besser werden. Beim ersten Mal ist es auch besser geworden." Nicht? Aber ist ja jetzt dann schlechter geworden. Und ohne künstliche Ernährung würde sie ja schon lange nicht mehr sein, bloß dann macht man sich ja auch Vorwürfe [...]"

$(A Z 5,109)$

Entlastung durch Verantwortungsabgabe

Viele Bezugspersonen geben Aufgaben, die sie vor Heimeinzug des Bewohners übernommen haben, etwa hinsichtlich der Medikamentenversorgung oder der Initialisierung von Arztbesuchen an das Pflegeheim ab. Durch die Verantwortungsabgabe hinsichtlich der medizinischen Versorgung, aber auch globaler gesehen, fühlen sich die Angehörigen entlastet.

„Das Positive im Moment ist, ich weiß, dass die Mama gut aufgehoben ist. Dass, [...] ich bin jetzt egoistisch vielleicht, aber dass ich jetzt auch mal wieder an mich denken kann, weil als ich die Mama die letzten zwei Jahre gepflegt habe, war ich nur bei der Mama. Wir haben gar nix mehrmachen können, mein Mann und ich, also nur Mutti, Mutti, Mutti. Das ist das Positive für mich, das hört sich jetzt vielleicht schlimm an, aber ich bin dann auch froh gewesen, dass ich die Verantwortung, [die] ich die ganze Zeit allein hatte, auch ein bisschen wegschieben konnte. Also auch aufs Heim ja. Da ging es mir dann auch besser."

$(B Z 6,198)$

\section{Persönlicher Benefit}

Das Einbezogensein der Bezugspersonen führt jedoch auch zu einem persönlichen Nutzen für Angehörige und Bewohner.

Bewohner bevorzugt Pflege durch Angehörige

So wird an mehreren Stellen deutlich, dass die Pflege durch Angehörige von den Bewohnern bevorzugt wird.

„Ja ja, was ich so machen kann ja. Ja dann ist ja mein Mann dann auch zufriedener, nicht, wenn ich das mache."

$(C Z 1,102)$

Zudem wird die Pflege durch die Angehörigen bisweilen besser akzeptiert.

„Also da gibt es [...] eine Familie, die kennen wir aber auch. Die haben vier Kinder da. Die haben das super organisiert, die kümmern sich auch immer um ihre Mutter und die füttern auch und die merken auch, dass die ganz anders isst, wenn jemand da ist."

$(B Z 1,112)$ 
Wertschätzung der Fürsorge durch den Bewohner

Auch für Angehörige ergibt sich ein persönlicher Benefit aus ihrem Einbezogensein, wenn ihr Handeln durch die Bewohner wertgeschätzt wird.

„Und dann verabschiede ich mich von ihm und dann bedankt er sich für alles, nicht."

$(C Z 1,16)$

\section{Beziehungsänderung zum Bewohner}

Häufig sind die Bezugspersonen als gesetzliche Betreuung für ihre pflegebedürftigen Angehörigen eingesetzt. Bedingt durch diese Stellvertreterrolle kann eine Beziehungsänderung zum Bewohner eintreten (AZ5, 109). Diese Stellvertretung zu übernehmen, fällt den Bezugspersonen mitunter nicht leicht. So wird mitunter gezögert, eine Betreuungsvollmacht zu übernehmen (AZ6, 10).

Zusammenfassend formuliert zeigt sich das Einbezogensein der Angehörigen an der medizinischen Versorgung im Pflegeheim durch das Übernehmen einiger Aufgaben. So werden neben der aktiven Hilfe bei der Pflege und Medikamentenbeschaffung zum Teil Hausarztbesuche initialisiert, Facharzttermine ausgemacht und Bewohner bei deren Praxisbesuchen begleitet. Darüber hinaus zeigt sich Einbezogensein durch Kontakt der Angehörigen zum Hausarzt oder den Versuch, aktiv auf die medizinische Versorgung der Bewohner Einfluss zu nehmen, wenn Unzufriedenheit besteht oder eine bislang noch nicht durchgeführte Versorgung des Bewohners gewünscht wird. Zum Teil erwerben die Angehörigen gezielt Wissen, um Aufgaben übernehmen zu können oder sind auch an der direkten pflegerischen Versorgung des Bewohners beteiligt. Auf der anderen Seite agieren die Angehörigen zurückhaltender, wenn die Versorgung in ihren Augen gewährleistet ist oder die Bewohner noch selbstständig sind. Die Gründe für das Einbezogensein der Angehörigen sind vielfältig und lassen sich mitunter auf ein Verantwortungsgefühl für den Bewohner oder auch den Wunsch des Bewohners nach Beteiligung der Angehörigen zurückführen. Aufgrund der Beteiligung entstehen für die Angehörigen stellenweise Belastungen, etwa durch die eingenommene Stellvertreterrolle. Auf der anderen Seite profitieren die Bezugspersonen etwa von der Wertschätzung ihres Handelns durch die Bewohner. 


\section{Diskussion}

Aus den Interviews gehen verschiedene Punkte hervor, die den privaten Bezugspersonen bei der medizinischen Versorgung ihrer pflegebedürftigen Angehörigen wichtig sind. Dazu gehört eine regelmäßige Präsenz der Hausärzte im Heim sowie generell die Gewährleistung einer zuverlässigen medizinischen Versorgung. Die Wichtigkeit eines persönlichen Kontakts zum Bewohner sowie dass die Hausärzte sich Zeit nehmen während ihrer Besuche, wird ebenfalls herausgestellt. Zudem legen die Bezugspersonen Wert darauf, dass Hausärzte auf ihre Anliegen eingehen und sie sich hinreichend über die Versorgung des Bewohners informiert fühlen. In die Zusammenarbeit zwischen Ärzten und Pflegekräften haben Angehörige häufig wenig Einblick, beschreiben diese dennoch oft positiv. Zum Teil wird das Verhältnis jedoch sehr hierarchisch erlebt. Mitunter übernehmen die Angehörigen innerhalb der medizinischen Versorgung einige Aufgaben und suchen bisweilen bei Gesprächsbedarf den Kontakt zum Hausarzt. In diesem Zusammenhang hat sich Einbezogensein als das zentrale Phänomen herausgestellt. Abhängig von der Versorgungswahrnehmung, gegebenen Heimstrukturen, einem Verantwortungsfühl oder dem Wunsch der Bewohner sind private Bezugspersonen unterschiedlich stark in die medizinische Versorgung der Heimbewohner involviert.

\subsection{Stärken und Schwächen}

Die Stärken der vorliegenden Untersuchung liegen methodisch bedingt in der offenen Zugangsweise. Hierdurch konnten, im Rahmen des vorher festgelegten Forschungsinteresses, die individuellen Perspektiven der Bezugspersonen zur medizinischen Versorgung von Pflegeheimbewohnern mit den von ihnen gewählten Schwerpunkten ermittelt werden. Es wurde jedoch deutlich, dass die medizinische Versorgung der Heimbewohner mitunter nicht im Zentrum des Interesses der privaten Bezugspersonen lag, sondern häufig auch deren pflegerischere Versorgung und der Alltag im Pflegeheim.

Bei der Auswahl der Interviewteilnehmer wurde keine statistisch repräsentative, sondern eine inhaltlich repräsentative Stichprobe angestrebt (Merkens 1997). Die 
Auswahl gemäß theoretischem Sampling (Strauss und Corbin 1996), im Sinne einer gezielteren Rekrutierung im Verlauf, zur Überprüfung bestehender Konzepte -etwa durch Kontrastierung gestaltete sich jedoch problematisch, da die Zahl der potentiellen Teilnehmer begrenzt war. Dementsprechend wurde die Rekrutierung vornehmlich anhand der Einschlusskriterien (Hauptbezugsperson, mindestens ein Kontakt pro Monat zum Pflegeheimbewohner, unterschriebene Einverständniserklärung, Volljährigkeit, ausreichende Deutschkenntnisse) vorgenommen.

Die Durchführung der Erhebung in drei verschiedenen Standorten, sowohl in städtischer als auch in ländlicher Umgebung, ermöglichte eine größere Vielfalt teilnehmender Bezugspersonen. Dennoch wurde der überwiegende Teil der Teilnehmer aus städtischem Gebiet rekrutiert.

Etwaige Verzerrungen in der Rekrutierung könnten dadurch entstanden sein, dass die potentiellen Studienteilnehmer in vielen Fällen von den Heimleitungen vorgeschlagen wurden. So ist es vorstellbar, dass hier vor allem engagierte Angehörige berücksichtigt wurden, die sich häufig im Pflegeheim aufhalten (Selektionsbias). Dies lässt sich auch anhand der ermittelten Besuchshäufigkeit, von durchschnittlich 16 Tagen im Monat, vermuten. Ein solches Vorgehen bei der Rekrutierung wurde gewählt, um die Anzahl potentieller Interviewteilnehmer innerhalb der Einschlusskriterien zu vergrößern, ohne dabei die Integrität des Pflegeheims etwa durch gezielte Rekrutierung durch Mitarbeiter der Studie zu verletzen. So war es Interviewvoraussetzung, dass sich auch das Pflegeheim zur Teilnahme an der Studie bereit erklärte. Entsprechend könnte hier eine positive Selektion dahingehend stattgefunden haben, dass nur Heime eingeschlossen wurden, welche hinsichtlich Verbesserungen und Entwicklung gegenüber aufgeschlossen sind oder auch nur keine Bedenken gegenüber Forschungsanliegen und Befragungen im eigenen Haus haben.

Da den Interviewteilnehmern keinerlei finanzielle Anreize geboten wurden, ist zudem von einem gewissen Interesse beziehungsweise einer Empfänglichkeit für den Forschungsgegenstand auszugehen. 


\subsection{Einordnung in die themenbezogene Literatur}

Es zeigen sich einige Berührungspunkte zwischen gewonnenen Ergebnissen in unserer Erhebung und der Literatur.

\section{Erfahrungen mit der medizinischen Versorgung}

Im Hinblick auf die medizinische Versorgung ihrer pflegebedürftigen Angehörigen ist den von uns interviewten Bezugspersonen wichtig, dass regelmäßig Hausarztbesuche stattfinden und verlässlich eine ärztliche Betreuung erfolgt. Hausärzte sollten sich nach Angehörigenmeinung bei ihren Besuchen Zeit für den Bewohner nehmen und einen persönlichen Kontakt pflegen. Bereits in der Untersuchung von Bemmann und Klewer 2012 führen die Autoren die Unzufriedenheit der befragten Angehörigen mit der ärztlichen Versorgung im Pflegeheim auf die seltenen Besuche der Hausärzte im Heim zurück.

Zur medizinischen Versorgung von Pflegeheimbewohnern gab es in den letzen Jahren mehrere Studien, die auch die Häufigkeit stattgefundener Arztkontakte untersuchten. So ergab sich in einem GEK-Report annähernd ein Behandlungsfall beim Hausarzt pro Heimbewohner pro Quartal (Rothgang et al. 2008). Hieraus lässt sich jedoch nicht auf die Kontakthäufigkeit pro Fall schließen. In einer anderen Erhebung (4.481 Bewohner in 58 Pflegeheimen) hatten circa $90 \%$ der stationär versorgten Pflegebedürftigen in den letzten vier Wochen einen persönlichen Hausarztkontakt (Schäufele et al. 2009). Aus diesen Studien ließe sich eine regelmäßige hausärztliche Versorgung der Heimbewohner ableiten, zur Art oder Dauer eines solchen Kontaktes wurden jedoch keine Angaben gemacht. Auf die Themen, die in unseren Interviews als wichtig beschrieben wurden, das Zeit nehmen beim ärztlichen Bewohnerbesuch und ein persönlicher Kontakt, lassen sich hier keine Rückschlüsse ziehen. In einer weiteren Studie berichteten Pflegedienstleitungen deutscher Pflegeheime mehrfach von zeitlich sehr begrenzten ärztlichen Visiten, häufig ohne direkten Kontakt zum Bewohner (eqsInstitut 2008). Diese Punkte schildern Angehörige auch in unserer Erhebung und kritisieren diese Vorgehensweise.

In den von uns geführten Interviews wird deutlich, dass regelmäßige, feste Besuchszeiten auch für die Angehörigen den Kontakt zum Arzt ihrer 
pflegebedürftigen Familienmitglieder erleichtern. In den letzten Jahren gab es in Deutschland zahlreiche Modellprojekte, die unter anderem eben jene regelmäßige Versorgung zum Ziel hatten. Es gibt mehrere Projekte, in denen Verträge zur Integrierten Versorgung entsprechend §140a-d SGB V zwischen Krankenkassen und verschiedenen zur Versichertenversorgung ermächtigten Leistungsträgern abgeschlossen wurden (Sozialgesetzbuch V, §140a-d 2015). Die Vertragspartner sind hier neben den Krankenkassen etwa einzelne Ärzte oder Arztnetze, Pflegeheimträger und Krankenhäuser mit dem Ziel einer interdisziplinärenfachübergreifenden medizinischen Versichertenversorgung (AOK- Bundesverband o.J.). Beteiligte Ärzte verpflichten sich unter anderem zu regelmäßigen hausärztlichen Visiten. Die Zeiträume variieren dabei in den einzelnen Projekten von wöchentlichen Heimbesuchen bis zu mindestens zwei Heimbesuchen/ persönlichen Kontakten pro Quartal je Versicherten (AOK Nordost 2015; Kassenärztliche Vereinigung Mecklenburg-Vorpommern und AOK Nordost 2011; Kassenärztliche Vereinigung Berlin, AOK Nordost, IKK Brandenburg und Berlin, Bahn BKK, Siemens-Betriebskrankenkasse 2011, AOK Baden-Württemberg 2013, Kassenärztliche Vereinigung Westfalen-Lippe, AOK Nordwest, BKKLandesverband Nordwest, IKK classic, Sozialversicherung für Landwirtschaft, Forsten und Gartenbau, Knappschaft, Ersatzkassen 2014, Dahlhoff 2011). In diesem Zusammenhang wäre es, sofern von den Angehörigen gewollt, wünschenswert, sie auch regelmäßiger in die ärztlichen Besuche einzubeziehen oder mindestens eine regelmäßigen Informationsweitergabe sicherzustellen.

\section{$\underline{\text { Interaktion }}$}

In den von uns geführten Angehörigeninterviews zeigt sich, dass Angehörige es schätzen, wenn sie sich vom Hausarzt hinreichend informiert fühlen, auch wenn zum Teil sehr unterschiedlicher Informationsbedarf besteht. Zudem ist es den Bezugspersonen wichtig, dass, wenn Anliegen für die Versorgung ihres pflegebedürftigen Angehörigen ihrerseits bestehen, der Hausarzt auf diese auch eingeht.

Aus einer amerikanischen Studie von Teno et al. 2001 geht hervor, dass Angehörige den Ärzten eine Schlüsselrolle in der Kommunikation von Informationen innen gegenüber zuschreiben, hier allerdings vor allem auf die 
Situation von Pflegeheimbewohnern am Lebensende bezogen. Aus unserer Erhebung ergibt sich, dass der Austausch von Informationen über die medizinische Versorgung des Bewohners auch häufig über die Pflegekräfte stattfindet, da der Hausarzt in der Regel schwerer erreichbar ist. Darüber hinaus ist der Hausarzt mitunter jedoch Ansprechpartner in belastenden Situationen den Bewohner betreffend für die Angehörigen.

In mehreren amerikanischen Studien ist mangelnder Kontakt zu den Ärzten im Pflegeheim eine Quelle für Unzufriedenheit seitens der privaten Bezugspersonen (Levy-Storms und Miller-Martinez 2005, Shield et al. 2005, Teno et al. 2004). Auch in den von uns geführten Interviews wird deutlich, dass die Bezugspersonen die Hausärzte bisweilen noch nie im Pflegeheim angetroffen haben oder zumindest Eigeninitiative seitens der Angehörigen für einen Kontakt zum Hausarzt nötig ist. Erleichtert wird die Kommunikation jedoch nicht selten dadurch, dass Bezugsperson und pflegebedürftiger Angehöriger von demselben Hausarzt betreut werden.

Die Wünsche der von uns interviewten Angehörigen für ihren eigenen Kontakt zu den Hausärzten beziehen sich vor allem auf die Kommunikation. Dies impliziert einen Informationsaustausch vom Arzt ausgehend über geplante und stattgefunden Visiten sowie besser über die Medikation des Bewohners in Kenntnis gesetzt zu werden. Ähnliche Wünsche formulierten Bezugspersonen von pflegebedürttigen Angehörigen in deutschen Pflegeheimen bereits in einer Untersuchung von Bemmann und Klewer 2012.

In einer kanadischen Studie von Caron et al. 2005 wünschten sich Angehörige von Demenzkranken am Lebensende häufigere offizielle Treffen mit den Professionellen, ohne sich dabei auf eine spezielle Berufsgruppe (Ärzte, Pflege ect.) festzulegen. Aus Angehörigensicht sollten diese Treffen dazu beitragen, den Krankheitsprozess ihrer Familienmitglieder besser zu verstehen, Fragen stellen zu können, Zweifel auszuräumen und das Gefühl von Einbeziehung zu erhalten (Caron et al. 2005). In den von uns geführten Interviews wird auch der Wunsch nach einer Kommunikation zu dritt beziehungsweise zu viert zwischen Angehörigen, Pflegekräften, Hausärzten und gegebenenfalls Bewohnern formuliert. Die Angehörigen erhoffen sich hiervon einen besseren 
Informationsaustausch beziehungsweise die Möglichkeit von Ab- und Aussprachen die Versorgung des Heimbewohners betreffend.

\section{Aufgaben der Angehörigen/ Einbezogensein in die medizinische Versorgung}

Einbezogensein der Bezugspersonen hat sich in unserer Erhebung als zentrales Phänomen hinsichtlich der medizinischen Versorgung der Heimbewohner herauskristallisiert. Mitunter übernehmen die Angehörigen hier einige Aufgaben und suchen bisweilen bei Gesprächsbedarf den Kontakt zum Hausarzt. Besteht Unzufriedenheit mit der medizinischen Versorgung des Bewohners, wird zum Teil versucht Einfluss zu nehmen. Wird die Versorgung als gewährleistet angesehen, agieren die Angehörigen zurückhaltender.

Bei Port et al. 2005 waren Angehörige von betreut wohnenden Bewohnern, die entsprechend keine 24- stündige Betreuung bekamen, häufiger in die medizinische Versorgung der Bewohner involviert, als Angehörige von Pflegeheimbewohnern. Dem ähnelnd haben einige Angehörige in unserer Erhebung mitunter vor Heimeinzug etwa Aufgaben hinsichtlich der Medikamentenversorgung übernommen, die Verantwortung diesbezüglich jedoch bei Heimeintritt an das Heim abgegeben.

Aus der Erhebung von Struppek 2010 geht hervor, dass die interviewten Angehörigen von kognitiv nicht beeinträchtigten Pflegeheimbewohnern generell selten und dann eher zu Beginn des Heimaufenthaltes des Bewohners Kontakt mit deren Ärzten hatten. In ähnlicher Weise wird auch in den von uns geführten Interviews als Grund für weniger Einbezogensein der Angehörigen in die medizinische Versorgung eine noch vorhandene Selbstständigkeit der Bewohner angegeben.

Aus mehreren Studien lässt sich entnehmen, dass sich Angehörige von Pflegeheimbewohnern teilweise als Fürsprecher dieser verstehen, um für die beste Versorgung der Bewohner zu sorgen. In unserer Erhebung lag für $73 \%$ der Heimbewohner eine gesetzliche Betreuung, in den meisten Fällen durch die befragten Angehörigen, vor. Diese gesetzliche Betreuung ist in Deutschland durch das Betreuungsrecht geregelt und wird durch das Betreuungsgericht eingesetzt, wenn Volljährige aufgrund einer geistigen, körperlichen oder seelischen Behinderung, wozu etwa auch die geistigen Folgen des Altersabbaus gezählt 
werden, ihre Angelegenheiten ganz oder teilweise nicht mehr selbstständig erledigen können. In solchen Fällen können eingesetzten Betreuern Aufgaben im Bereich der Gesundheitssorge etwa bezüglich Entscheidungen hinsichtlich Untersuchung des Gesundheitszustandes, Heilbehandlung oder ärztlicher Eingriffe der betreuten Person übertragen werden. Eine gerichtliche Genehmigung ist nicht notwendig, solange zwischen Betreuer und behandelndem Arzt Einvernehmen darüber besteht, dem Willen des Betreuten zu entsprechen (Bundesministerium der Justiz und für Verbraucherschutz 2015).

Dieser Fürsorgerolle entsprechend fürchteten Angehörige in einer amerikanischen Studie, dass die Grundbedürfnisse der Bewohner vernachlässigt würden, wenn sie selbst nicht anwesend wären (Teno et al. 2001). Und auch in einer anderen Erhebung waren einige Bezugspersonen davon überzeugt, dass ihre Besuche einen Einfluss auf die Aufmerksamkeit des Personals für den Bewohner hätten (Tsai und Tsai 2012). Die hier beschriebenen Erhebungen beziehen sich dabei vor allem auf die pflegerische Versorgung. In den von uns geführten Interviews werden Zweifel, ob die Versorgung ohne Angehörigenpräsenz schlechter wäre, sowohl auf die pflegerische als auch auf die medizinische Versorgung bezogen, geäußert. Etwa werden Zweifel bekundet, ob Bewohner ohne Angehörige bei Bedarf immer eine ärztliche Versorgung erhalten würden.

Wetle et al. beschrieben 2005, dass Angehörige immer dann zu „Anwälten“ (Wetle et al. 2005 S. 646) ihrer umsorgten Pflegeheimbewohner wurden, wenn sie die pflegerische, aber auch die medizinische Versorgung als nicht zufriedenstellend empfanden. Diese Anwaltsfunktion beinhaltete etwa, dass Angehörige bei gesundheitlichen Veränderungen des Bewohners gegenüber dem Personal für eine Versorgung eintraten. In ähnlicher Weise werden auch bei Keefe und Fancey 2000 Familienmitglieder als Anwälte ihrer pflegebedürftigen Angehörigen bezeichnet, wenn diese die Interessen der Bewohner, hier eher bezogen auf die pflegerische Versorgung, vertraten. Sandberg et al. 2001 beschrieben ebenfalls hinsichtlich der pflegerischen Versorgung, dass Angehörige bei einer schlechten Versorgungswahrnehmung ihre Aufmerksamkeit diesbezüglich erhöhten. Auch in unserer Erhebung üben Angehörige bisweilen eine gewisse Kontrollfunktion aus. Dies ist jedoch nicht ausschließlich auf die Versorgungswahrnehmung zurückzuführen, sondern etwa auch auf ein Verantwortungsgefühl dem pflegebedürftigen Angehörigen gegenüber. Zudem erstreckt sich die 
Kontrollfunktion bisweilen auch auf die medizinische Versorgung. So versuchen Angehörige mitunter, einen Überblick über die Medikation des Bewohners zu behalten, drängen bei subjektivem Bedarf auf Krankenhauseinweisungen oder sind an Hausarztwechseln aufgrund einer schlechten Versorgungswahrnehmung beteiligt. Diese Kontrollfunktion möchten in unserer Erhebung aber längst nicht alle Angehörigen übernehmen. So gibt es Bezugspersonen, die bewusst zu ihrer Entlastung die Verantwortung bezüglich der medizinischen Versorgung an das Heim abgeben und beispielsweise nur noch eingeschränkt darüber informiert werden wollen.

Einen möglichen Zusammenhang zwischen einer schlechten Versorgung und vermehrter Anwesenheit von Angehörigen im Pflegeheim erwägen Grabowski und Mitchell 2009 in ihrer Studie. Ein solcher Zusammenhang lässt sich in unserer Erhebung nicht überprüfen. Dennoch involvieren sich die von uns interviewten Angehörigen bisweilen mehr, wenn dies subjektiv zu einer Verbesserung der medizinischen Versorgung beiträgt oder sie diese gefährdet sehen.

Seit 2002 können sich Angehörige auch aktiv im Heimbeirat für die Rechte der Pflegeheimbewohner einsetzen. Seit diesem Zeitpunkt dürfen sie Mitglieder in den Heimbeiräten sein und Themen wie Unterkunft, Aufenthaltsbedingungen, Heimordnung, Betreuung, Verpflegung und Freizeitgestaltung der Pflegeeinrichtung mitgestalten. Die Mitwirkung erstreckt sich zudem auf die Sicherstellung einer gebührenden Qualität der Betreuung im Heim und auf Verhandlungen zu Leistungs-, Qualitäts-, Vergütungs- und Prüfvereinbarungen (LQVP-Vereinbarungen) der Pflegeeinrichtung (Heimgesetz, § 10 Abs. 1, 5 2009). In einer Studie zur Heimmitwirkung wurden die Tätigkeitsbereiche der Heimbeiräte erfasst und festgestellt, dass diese vor allem bei der Alltags-, Freizeit und Verpflegungsgestaltung mitwirken. Über Verhandlungen zu LQVP-Vereinbarungen wurden die Heimbeiräte in 2/3 der Fälle zwar informiert, nahmen jedoch kaum selbst daran teil (Mingot et al. 2007). Um die Position der Angehörigen zu stärken, wäre es wünschenswert, das Tätigkeitsspektrum der Heimbeiräte weiter auszubauen. 


\subsection{Schlussfolgerungen und Ausblick}

In Deutschland hat sich seit 1999 die Zahl der in Pflegeheimen vollstationär betreuten Pflegebedürftigen um rund $36 \%$ erhöht (Statistisches Bundesamt 2015), sodass zum Jahresende 2013 laut Statistischem Bundesamt etwa 764.000 Menschen in Pflegeeinrichtungen versorgt wurden. Durch die zunehmende vollstationäre Versorgung der Pflegebedürftigen wächst auch die Gruppe der Angehörigen von Bewohnern im Pflegeheim. Diese stellen eine wichtige Personengruppe im Setting Pflegeheim dar, die sich häufig intensiv zum Wohle des Bewohners einsetzen.

Diese wesentliche Funktion sollte von den anderen Akteuren im Heim mehr Beachtung erfahren. Nicht zuletzt aufgrund der steigenden Anzahl von Bewohnern mit Demenz werden Angehörige voraussichtlich zunehmend zu Fürsprechern werden und Einfluss ausüben wollen. Die Einrichtung von organisatorischen Strukturen im Heim, z. B. durch die selbstverständliche Aufstellung eines Angehörigenkreises und die zunehmende unaufgeforderte Berücksichtigung von Angehörigen in medizinische Entscheidungen (bei Einverständnis des Bewohner) wäre wünschenswert. Es erscheint hier ebenso erstrebenswert, die Position von Angehörigen etwa in Heimbeiräten zu stärken sowie sie inhaltlich in Angehörigengruppen zu unterstützen. Die Akzeptanz und Einbeziehung durch die beteiligten Akteure und die Politik sollte zudem gefördert werden.

Bei der medizinischen Versorgung von Heimbewohnern übernehmen Angehörige viele Funktionen und fühlen sich oft emotional belastet. Entlastung kann beispielsweise erfolgen indem bewusst Verantwortung an das Heim abgegeben wird, wenn die medizinische Versorgung als angemessen eingestuft wird. Erst eine kürzlich erschienene Studie des Forsa Instituts weist auf, dass ihre Angehörige zuhause pflegende Personen zu einem Drittel schon an depressiven Symptomen gelitten haben (Compass Private Pflegeversicherung 2015). Zu Angehörigen von Pflegeheimbewohnern in Deutschland gibt es aktuell keine Zahlen. Unsere Ergebnisse lassen allerdings vermuten, dass eine stärkere belastende emotionale Einbeziehung bei einigen von innen ebenfalls vorliegt. Was es für sie selbst bedeutet, wenn ihr Angehöriger in das Heim zieht, haben wir in unserer Untersuchung nicht erfasst, da der Schwerpunkt mehr bei dem Blick auf die medizinische Versorgung des Bewohners lag. Es wäre sinnvoll, die Situation 
von Angehörigen vor, bei und nach dem Heimeinzug ihrer Angehörigen weiter zu untersuchen und die wahrscheinlich belastende Situation in der primärmedizinischen Versorgung zu berücksichtigen, etwa durch Beratungsangebote.

Aus unserer Erhebung geht zudem hervor, dass Angehörige von Pflegeheimbewohnern für diese eine patientenzentrierte medizinische Versorgung, bei der sich der Hausarzt Zeit für einen persönlichen Kontakt zu seinen Patienten nehmen kann, wünschen. Entsprechend erscheint es für die Zukunft erstrebenswert, dass Hausärzte gezielte Freiräume für die Betreuung von Pflegeheimbewohnern schaffen. Wünschenswert wären etwa feste Besuchs- bzw. Sprechzeiten in den Heimen, die auch für Angehörige den Kontakt zum Hausarzt erleichtern würden. Zudem scheint aus Angehörigenperspektive eine Zunahme von festen Anbindungen bestimmter Hausärzte an die Pflegeheime erstrebenswert, um eine kontinuierlichere Versorgung zu gewährleisten. Dies würde auch dem aus unserer Erhebung hervorgehenden Wunsch der Angehörigen nach einer regelmäßigen hausärztlichen Versorgung gerecht werden.

Diesbezüglich gab es in den letzten Jahren einige positive Entwicklungen mit einer Reihe bereits in der Diskussion erwähnter Modellprojekte. Diese Projekte sind jedoch bis dato nicht flächendeckend realisiert, weshalb für die Zukunft eine breitere Umsetzung solcher Versorgungsstrukturen mit regelmäßigen, festen Hausarztvisiten erstrebenswert erscheint. Folglich wäre es ein interessanter Ansatz für zukünftige Forschungen, von Pflegeheimbewohnern und Angehörigen zur Auswirkung regelmäßiger hausärztlicher Visiten auf die medizinische Versorgung und deren Wahrnehmung zu befragen.

Ein darüber hinaus wünschenswerter künftiger Forschungsansatz wäre eine quantitative Studie zur medizinischen Versorgung der Pflegeheimbewohner, die die Perspektive der Angehörigen und deren Einbezogensein in die medizinische Versorgung, möglicherweise sogar repräsentativ abbildet.

Angehörige sind eine wichtige Ressource im Pflegeheim, deren Wert aktuell noch nicht hinreichend erkannt zu sein scheint. Diese Doktorarbeit stellt diesen Wert dar und trägt hoffentlich dazu bei, die Relevanz der Angehörigenperspektiven und die Unterstützung durch Angehörige im Alltag des Heimes publik zu machen. Das 
übergeordnete Ziel wäre es, Angehörige so in die Heimarbeit einzubeziehen, dass letztlich der Bewohner hinsichtlich der medizinischen Versorgung profitiert. 


\section{$5 \quad$ Zusammenfassung}

Einleitung: In Deutschland wird die steigende Zahl pflegebedürftiger Menschen zunehmend in stationären Einrichtungen versorgt. Dieser Zuwachs bringt neue Herausforderungen, nicht zuletzt für die medizinische Versorgung der Pflegebedürftigen, mit sich. Qualitative Interviews mit beteiligten Akteuren können Erkenntnisse zum „Status quo“ liefern. Die vorliegende Arbeit beschäftigt sich mit der Perspektive der Angehörigen, da diese häufig in die medizinische Versorgung eingebunden sind und einen differenzierten Blick auf die Versorgungsqualität haben.

Fragestellung: Welche Erfahrungen haben Angehörige im Pflegeheim mit der medizinischen Versorgung gemacht und wie wird die Zusammenarbeit zwischen Ärzten und Pflegekräften wahrgenommen? Welche Aufgaben übernehmen Angehörige bei der medizinischen Versorgung?

Methoden: Die Arbeit ist eingebettet in die qualitative, multizentrische Studie „INTERPROF - Interprofessionelle Zusammenarbeit und Kommunikation im Pflegeheim". In drei Regionen Deutschlands wurden hier unter anderem 23 offene Leitfadeninterviews mit 27 privaten Bezugspersonen in 14 Pflegeheimen durchgeführt. Die Interviews wurden, angelehnt an die Methode der Grounded Theory, ausgewertet.

Ergebnisse: Als zentrales Phänomen wurde das „Einbezogensein“ der Angehörigen in die medizinische Versorgung erarbeitet. Angehörige versuchen, aktiv auf die medizinische Versorgung der Bewohner Einfluss zu nehmen. So werden neben der aktiven Hilfe bei der Pflege und Medikamentenbeschaffung zum Teil Hausarztbesuche initialisiert, Facharzttermine ausgemacht und Bewohner bei deren Praxisbesuchen begleitet. Darüber hinaus zeigt sich Einbezogensein durch den Kontakt der Angehörigen zum Hausarzt. Die Gründe hierfür sind vielfältig und lassen sich etwa auf ein Verantwortungsgefühl für den 
Bewohner oder auch den Wunsch des Bewohners nach Beteiligung der Angehörigen zurückführen. Angehörige üben zum Teil eine gewisse „Kontrollfunktion“ aus, dies führt mitunter zu emotionaler und zeitlicher Belastung. Es vermittelt andererseits eine gewisse Sicherheit, über Vorgänge informiert zu sein und diese beeinflussen zu können. Angehörige sind jedoch weniger „einbezogen“ oder wollen weniger „einbezogen“ werden, wenn sie die medizinische Versorgung gewährleistet sehen oder die Bewohner noch selbstständig sind. Folglich fühlen sie sich durch die bewusste Verantwortungsabgabe an die Pflegeeinrichtung entlastet.

Schlussfolgerungen: Angehörige von Pflegeheimbewohnern stellen eine wichtige Personengruppe im Pflegeheim dar und bringen sich häufig intensiv, auch hinsichtlich der medizinischen Versorgung, zum Wohle der Bewohner ein. Wesentliche Anliegen sind eine patientenzentrierte, regelmäßige medizinische Versorgung. Es erscheint erstrebenswert, die Akzeptanz und Einbeziehung der Angehörigen durch die beteiligten Akteure im Heim und die Politik zu fördern. Auch die Einrichtung von organisatorischen Strukturen im Heim, z. B. durch die selbstverständliche Aufstellung eines Angehörigenkreises und die zunehmende unaufgeforderte Berücksichtigung von Angehörigen in medizinischen Entscheidungen (bei Einverständnis des Bewohner) wäre wünschenswert. Zudem sollte ihre Position in Heimbeiräten gestärkt werden. Das übergeordnete Ziel wäre es, Angehörige so in die Heimarbeit einzubeziehen, dass letztlich der Bewohner hinsichtlich der medizinischen Versorgung profitiert. 


\subsection{Summary}

Introduction: The rising number of persons needing care is increasingly being catered for by means of in-patient facilities in Germany. This growth brings new challenges with it, not least where medical care for the residents of nursing homes is concerned. Qualitative interviews with the stakeholders can provide insights into the status quo. This dissertation deals with the perspective of the relatives, who are often involved in the medical care and have a differentiated viewpoint on the quality of care.

Questions: What are the experiences of the relatives of care home patients where medical care is concerned and how do they perceive the working partnership between physicians and care staff? Which of the tasks involved in medical care are performed by relatives?

Methods: The dissertation is part of the qualitative, multi-centric study "INTERPROF - Interprofessionelle Zusammenarbeit und Kommunikation im Pflegeheim" (Interprofessional Working Partnerships and Communication in Nursing Homes). As part of the dissertation, 23 open-ended, guided interviews were conducted with 27 private individual stakeholders in 14 nursing homes in three regions of Germany. The interviews were evaluated based on the grounded theory method.

Results: The involvement of relatives in medical care was deemed to be a key phenomenon. Relatives actively try to influence the medical care provided to residents. For example, they actively help with care and obtaining medicine and some of them initiate visits by family physicians, make appointments with consultants and accompany residents on their visits to practices. Involvement is also reflected in relatives' contact with family physicians. The reasons are varied and can be attributed to a feeling of responsibility for the resident or the resident's wish for the relatives to be involved, for example. Some relatives have a certain monitoring role which results inter alia in an emotional burden and can be very time consuming. It also conveys a certain reassurance that they have been 
informed of events and are able to influence them. Relatives are less involved, or want to be less involved, however, when they see for certain that medical care is being provided or the residents are still independent. They therefore feel that their load is reduced by their conscious decision to hand over responsibility to the care facility.

Conclusions: Relatives of nursing home residents are important stakeholders when it comes to nursing homes and are frequently intensely involved in ensuring the wellbeing of the residents, including with regard to medical care. Patientcentric, regular medical care is a major concern. It appears desirable for decisionmakers in the homes and in the political arena to promote the acceptance and involvement of relatives. Establishing organizational structures in the home, for example by setting up a relatives group as a matter of course and giving greater consideration to relatives when it comes to medical decisions without this needing to be requested (given the consent of the resident) would also be well received. Moreover, their position on nursing home councils should be strengthened. The overarching aim would be to involve relatives in the work performed in nursing homes so that, ultimately, residents see benefits in terms of their medical care. 


\section{Anhang}

\subsection{Anlage 1: Information für Bezugspersonen zur Teilnahme an der Studie}

\section{Interprofessionelle Zusammenarbeit und Kommunikation im Pflegeheim}

Projektleitung:

Prof. Dr. med. Eva Hummers-Pradier und Dr. med. Christiane Müller, MPH Institut für Allgemeinmedizin der Universitätsmedizin Göttingen

Humboldtallee 3 8

D-37073 Göttingen

Information für Bezugspersonen zur Teilnahme an der Studie

„Interprofessionelle Zusammenarbeit und Kommunikation im Pflegeheim“

Sehr geehrte Damen und Herren,

die medizinische Versorgung von Pflegeheimbewohnem wird von verschiedenen Berufsgruppen, hauptsächlich der Pflege und von Ärzten, geleistet. Wir wollen in unserer Studie mehr über die Sichtweisen und Bedürfnisse aller Beteiligten erfahren sowie untersuchen, wie sich die Zusammenarbeit und Kommunikation gestalten kann. Letztlich wollen wir einen Beitrag dazu leisten, die medizinische Versorgung von Pflegeheimbewohnem zu stärken.

Deshalb würden wir gerne mit Bewohnem, Bezugspersonen, Ärtten und Pflegekräften persönliche Gespräche (Interviews) führen. In diesen geht jeweils um ihre Perspektive und persönlichen Erfahrungen zur Zusammenarbeit der Berufsgruppen im Pflegeheim. Während des Interviews können Sie jederzeit eigene Themen ansprechen sowie Sie auch das Gespräch jederzeit unterbrechen oder abbrechen können. Das Interview findet an einem Ort und zu einer Zeit Ihrer Wahl statt und dauert bis zu einer Stunde. Die Teilnahme an dieser Studie ist freiwillig, eine Nichtteilnahme bringt keinedlej Nachteile mit sich. Da es sich um ein wissenschaftiches Forschungsprojekt handelt, werden Sie voraussichtlich keinen unmittelbaren Nutzen erfahren.

Das Interview wird auf Tonband aufgezeichnet, um es im Anschluss wissenschaftich auswerten zu können. Nach Beendigung der Studie wird es zwar archiviert, aber nicht für weitere Studien oder im Unterricht für die Studierenden verwendet. Die Auswertung des Interviews sowie des Kurzfragebogens erfolgt pseudonymisiert, d.h. ohne Verwendung Ihres Namens oder Ihrer persönlichen Daten; Rückschlüsse auf Ihre Person werden daher nicht mōglich sein.

Entsprechend den Regeln guter wissenschaftlicher Praxis werden Ihre Daten sowie die Aufnahme für einen Zeitraum von 10 Jahren unter den Bedingungen des Datenschutzes gespeichert, eine Weitergabe an Dritte (z.B. an ihren Vorgesetzten) ist ausgeschlossen Sie kōnnen Ihr einmal gegebenes Einverständnis jederzeit ohne Angabe von Grūnden zurūckziehen, ohne dass Ihnen dadurch irgendwelche Nachteile entstehen. Alle bis dahin erhobenen Daten (einschließlich der angefertigten Interviewtranskription) sowie die Audioaufnahmen im Original und der DVD als Sicherheitskopie würden in diesem Fal unverzüglich vernichtet werden.

Sofern Sie noch Fragen haben, beantworte ich diese gerne.

Dr. med. Christiane Müller, MPH

Wissenschaftliche Mitarbeiterin

Tel.: 0551/39226921 


\subsection{Anlage 2: Einverständniserklärung zur Teilnahme an der Studie}

\section{Demografische Angaben Bezugsperson}

Methode

$\square$
$\square$
$ㅁ$
$\square$
Interview
Teilnehmende Beobachtung
Fokusgruppe
Expertenworkshop

Datum:

\section{Einrichtungsträger akommunal/öffentlich afrei-gemeinnützig aprivat}

Größe der Einrichtung Plātze

1. Alter. Jahre

2. Geschlecht:
口 mānnlich
$\square$ weiblich

3. In welchem Verhältnis stehen Sie zum Bewohner?
- Sohn/Tochter
$\square$ Enkel/Enkelin
a Ehemann/Ehefrau
口 anderer Verwandtschaftsgrad, nāmlich

口 keinem Verwandtschaftsgrad, sondern

4. Existiert eine gesetzliche Betreuung für den Bewohner?
口 ja
a nein
$\square$ ist beantragt
falls ja

Sind Sie der gesetzliche Betreuer?

口 ja

a nein

5. Wie lange lebt der Bewohner bereits in dieser Einrichtung? Jahre

6. Wie häufig besuchen Sie den Bewohner pro Monat?

7. Wieviel Zeit verbringen Sie durchschnittlich im Monat beim Bewohner? Stunden

8. Welches ist lhr höchster Schulabschluss?

9. Welchen Beruf/Tätigkeit üben Sie aus?

10. Nur bei Beobachtungen: Was ist der Grund der heutigen Konsultation? 


\subsection{Anlage 3: Demografiebogen}

\section{Demografische Angaben Bezugsperson}

\section{interpro:}

\begin{tabular}{|c|c|c|c|}
\hline Methode & $\begin{array}{l}\square \\
\square \\
\square \\
\square\end{array}$ & $\begin{array}{l}\text { Interview } \\
\text { Teilnehmende Beobachtung } \\
\text { Fokusgruppe } \\
\text { Expertenworkshop }\end{array}$ & $\begin{array}{l}\text { Einrichtungsträger } \\
\text { qkommunal/öffentlich } \\
\text { qfrei-gemeinnützig } \\
\text { aprivat }\end{array}$ \\
\hline Datum: & & & $\begin{array}{r}\text { Größe der Einrichtung } \\
\text { Plātze }\end{array}$ \\
\hline 1. Alter: & Jahre & & $\begin{array}{l}\text { 口 Stadt ( }>5000 \text { Einwohner) } \\
\text { a Land (<5000 Einwohner) }\end{array}$ \\
\hline
\end{tabular}

2. Geschlecht:
c mānnlich
$\square$ weiblich

3. In welchem Verhältnis stehen Sie zum Bewohner?
口 Sohn/Tochter
口 Enkel/Enkelin
c anderer Verwandtschaftsgrad, nāmlich_
- keinem Verwandtschaftsgrad, sondern
口 ja
$\square$ nein
$\square$ ist beantragt
falls ja
Sind Sie der gesetzliche Betreuer?
口ja $\mathrm{ja}$ nein

口 Ehemann/Ehefrau

4. Existiert eine gesetzliche Betreuung für den Bewohner?

5. Wie lange lebt der Bewohner bereits in dieser Einrichtung? Jahre

6. Wie häufig besuchen Sie den Bewohner pro Monat?

7. Wieviel Zeit verbringen Sie durchschnittlich im Monat beim Bewohner? Stunden

8. Welches ist lhr höchster Schulabschluss?

9. Welchen Beruf/Tätigkeit üben Sie aus?

10. Nur bei Beobachtungen: Was ist der Grund der heutigen Konsultation? 


\subsection{Anlage 4: Interviewleitfaden}

Themenbereiche für die offenen Leitfadeninterviews Hauptbezugsperson

1. Erzählung eines typischen Besuchs im Pflegeheim

Uns interessieren Ihre eigenen Erfahrungen. Wie ist das denn so bei Ihren Besuchen im Pflegeheim üblicherweise? Erzählen Sie mal...

Was sind so typische Situationen, wenn Sie an die Pflege Ihres Angehörigen denken?

Wie ist das so mit den Pflegekräften? Wenn Sie da Kontakt haben oder etwas besprechen...?

Und was fällt Ihnen ein, wenn Sie die Kontinuität in der medizinischen Versorgung betrachten? Gibt es die? Wird Ihr Angehöriger beispielsweise noch von anderen Ärzten versorgt? Erzählen Sie...

Beschreiben Sie mal einen typischen Hausarztbesuch. Wie läuft der für gewöhnlich ab? Erzählen Sie mal...

Und können Sie sich auch an Hausbesuche erinnern, die ungewöhnlich waren?

Wie ist das üblicherweise, wenn Ihr Angehöriger mal einen Arzt braucht?

\section{Beschreibung des letzten Hausarztbesuchs}

Können Sie sich noch an den letzten Besuch des Hausarztes bei Ihrem Angehörigen erinnern?

2a) Hauptbezugsperson dabei gewesen

Wie war das denn so? Erzählen Sie doch mal...

Wie ist es eigentlich dazu gekommen, dass der Arzt ihn besucht hat?

Wie ist der Hausarztbesuch denn so abgelaufen? 
Wie haben Sie den Hausarztbesuch bei Ihrem Angehörigen erlebt?

Was passierte nach dem Hausbesuch? Wie ging es weiter?

2b) Hauptbezugsperson nicht dabei gewesen

\section{Was hat Ihnen Ihr Angehöriger darüber erzählt?}

Wie ist es eigentlich dazu gekommen, dass der Arzt ihn besucht hat? Was hat Ihr Angehöriger darüber berichtet?

Wie ist der Hausarztbesuch denn so abgelaufen? Was hat Ihnen Ihr Angehöriger darüber erzählt?

Was passierte nach dem Hausbesuch?

3. Erfahrungen mit Zuständigkeitsbereichen und Aufgabenverteilungen

Was machen im Pflegeheim Ihres Angehörigen denn so die Ärzte und was machen die Pflegekräfte? Wofür sind die denn so zuständig?

Welche Aufgaben übernimmt Ihr Angehöriger denn so selbstständig?

Welche Aufgaben übernehmen Sie dabei?

Wie arbeiten Ärzte und Pflegekräfte Ihrer Erfahrung nach zusammen?

4. Vorstellungen und Phantasien über die ideale Situation/Versorgung im Pflegeheim

Lassen Sie Ihrer Phantasie freien Lauf. Wie sähe in Ihren Augen das ideale Pflegeheim aus? Was würden Sie sich da wünschen?

Wie würden Sie sich die ideale Versorgung vorstellen? Erzählen Sie... 


\section{$7 \quad$ Literaturverzeichnis}

Balzer K, Butz S, Bentzel J, Boulkhemair D, Lühmann D: Beschreibung und Bewertung der fachärztlichen Versorgung von Pflegeheimbewohnern in Deutschland (Schriftenreihe Health Technology Assessment Band 125). 1. Auflage; o. Verl., Köln 2013

Bemmann R, Klewer J (2012): Angehörigenbefragung in einer stationären Altenpflegeeinrichtung. HBScience $\underline{3}, 126-130$

Berglund A (2007): Satisfaction with caring and living conditions in nursing homes: Views of elderly persons, next of kin and staff members. Int J Nurs Pract $\underline{13}, 46-51$

Böhm A: Theoretisches Codieren: Textanalyse in der Grounded Theory. In: Flick U, Kardorff E, Steinke I (Hrsg.): Qualitative Forschung - Ein Handbuch. Rowohlt Taschenbuch Verlag, Reinbek 2012, 475-485

Born A, Weigel R (2000): Was lässt sich verbessern? Handlungsoptionen auf der Basis fundierter Daten ermitteln und umsetzen. Altenheim $\underline{39}, 18-25$

Busch S, Weber P, Becker W: Projekt ZuBA. Zufriedenheit von Bewohnern und Angehörigen/Betreuern in stationären Altenpflegeeinrichtungen. Abschlussbericht. o.Verl., Hamburg 2006

Caron CD, Griffith J, Arcand M (2005): Decision Making at the End of Life in Dementia: How Family Caregivers Perceive Their Interactions With Health Care Providers in Long-Term-Care Settings. J Appl Gerontol 24, 231-247

Dahlhoff G: AOK- Pflegenetze: AOK Bayern - Die Gesundheitskasse. 12. Sitzung des Landesgesundheitsrates. Zitiert nach Inhaltsangabe des Vortrages (gehalten 06.06.2011, München)

Engels D, Pfeuffer F: Die Einbeziehung von Angehörigen und Freiwilligen in die Pflege und Betreuung in Einrichtungen. In: Schneekloth U, Wahl HW (Hrsg.): Integrierter Abschlussbericht. Möglichkeiten und Grenzen selbstständiger Lebensführung in stationären Einrichtungen (MuG IV) - Demenz, Angehörige und Freiwillige, Versorgungssituation, sowie Beispielen für „Good Practice“. o.Verl., München 2007, 233-299

Eskildsen M, Price T (2009): Nursing home care in the USA. Geriatr Gerontol Int $\underline{9}, 1-6$

Flick U, Kardorff E, Steinke I (Hrsg.): Qualitative Forschung - Ein Handbuch. Rowohlt Taschenbuch Verlag, Reinbek 2012

Friebertshäuser B, Prengel A (Hrsg.): Handbuch Qualitative Forschungsmethoden in der Erziehungswissenschaft. Juventa Verlag, Weinheim 1997 
Gasquet I, Dehé S, Gaudebout P, Falissard B (2003): Regular Visitors Are Not Good Substitutes For Assessment Of Elderly Patient Satisfaction With Nursing Home Care And Services. J Gerontol A-Biol $\underline{58 A}$, 1036-1041

Givens JL, Kiely DK, Carey K, Mitchell SL (2009): Healthcare Proxies of Nursing Home Residents with Advanced Dementia: Decisions They Confront and Their Satisfaction with Decision-Making. J Am Geriatr Soc 푸, 1149-1155

Givens JL, Lopez RP, Mazor KM, Mitchell SL (2012): Sources of Stress for Family Members of Nursing Home Residents With Advanced Dementia. Alzheimer Dis Assoc Disord 26, $254-259$

Grabowski DC, Mitchell SL (2009): Family oversight and the quality of nursing home care for residents with advanced dementia. Med Care $\underline{47}, 568-574$

Guba EG, Lincoln YS: Effective evaluation (Jossey-Bass social and behavioral science series). 1st ed; Jossey-Bass Publishers, San Francisco 1981

Hallauer JF, Bienstein C, Lehr U, Rönsch H: SÄVIP - Studie zur ärztlichen Versorgung in Pflegeheimen. VINCENTZ NETWORK Marketing Service, Hannover 2005

Hasseler M, Wolf-Ostermann K: Wissenschaftliche Evaluation zur Beurteilung der PflegeTransparenzvereinbarungen für den ambulanten (PTVA) und stationären (PTVS) Bereich. o.Verl., 0.O. 2010

Hildenbrand B: Anselm Strauss. In: Flick U, Kardorff E, Steinke I (Hrsg.): Qualitative Forschung - Ein Handbuch. Rowohlt Taschenbuch Verlag, Reinbek 2012, 32-37

Hoffmann PM, Korte MT: Betreuungsrechtliche Praxis in Einrichtungen der stationären Altenhilfe (Schriftenreihe des Bundesministeriums für Familie, Senioren, Frauen und Jugend Band 223). W. Kohlhammer, Stuttgart 2002

Hopf C (1978): Die Pseudo-Exploration: Überlegungen zur Technik qualitativer Interviews in der Sozialforschung. Z Soziol $\underline{7}, 97-115$

Hopf C: Qualitative Interviews - ein Überblick. In: Flick U, Kardorff E, Steinke I (Hrsg.): Qualitative Forschung - Ein Handbuch. Rowohlt Taschenbuch Verlag, Reinbek 2012, 355-357

Keefe J, Fancey P (2000): The Care Continues: Responsibility for Elderly Relatives before and after Admission to a Long Term Care Facility. Fam Relat $\underline{49}$, 235-244

Kelle U: Computergestützte Analyse qualitativer Daten. In: Flick U, Kardorff E, Steinke I (Hrsg.): Qualitative Forschung - Ein Handbuch. Rowohlt Taschenbuch Verlag, Reinbek 2012, 485-502

Kriz D, Schmidt J, Nübling R (2006): Zufriedenheit von Angehörigen mit der Versorgung in stationären Altenpflegeeinrichtungen. Entwicklung des Screening-Fragebogens ZUFA-7. Pflege $\underline{19}$, 88-96 
Lavizzo-Mourey RJ, Zinn J, Taylor L (1992): Ability of Surrogates To Represent Satisfaction of Nursing Home Residents with Quality of Care. J Am Geriatr Soc $\underline{40}$, 39-47

Legewie H: Vorwort zur deutschen Ausgabe. In: Strauss A, Corbin J (Hrsg.): Grounded Theory: Grundlagen Qualitativer Sozialforschung. Beltz Psychologie Verlags Union, Weinheim 1996, XII-XIII

Levy-Storms L, Miller-Martinez D (2005): Family Caregiver Involvement and Satisfaction With Institutional Care During the 1st Year After Admission. J Appl Gerontol 24, 160174

Merkens H: Stichproben bei qualitativen Studien. In: Friebertshäuser B, Prengel A (Hrsg.): Handbuch Qualitative Forschungsmethoden in der Erziehungswissenschaft. Juventa Verlag, Weinheim 1997, 97-106

Merton RK, Fiske M, Kendall PL: The Focused Interview. A manual of problems and procedures. 2nd ed; The Free Press, New York 1990

Mingot K, Ritter J, Stiehr K: Forschungsprojekt "Evaluation der Heimmitwirkung". Endbericht an das Bundesministerium für Familie, Senioren, Frauen und Jugend Referat 302. o.Verl., Bonn 2007

Müller CA, Tetzlaff B, Theile G, Fleischmann N, Cavazzini C, Geister C, Scherer M, Weyerer S, van den Bussche H, Hummers-Pradier E (2015): Interprofessional collaboration and communication in nursing homes: a qualitative exploration of problems in medical care for nursing home residents - study protocol. J Adv Nurs $\underline{71}$, $451-457$

Mutscher W, Prehm K, Wosegien A (2000): Aus Kundensicht. Eine Befragung von Zufriedenheit von Bewohnern und Angehörigen. Altenheim 1, 28-33

Nübling R, Kriz D, Kress G, Schremmp C, Löschmann C, Schmidt J (2004a): Angehörigenbefragungen - Potenziale für das interne Qualitätsmanagement in Altenpflegeeinrichtungen. Gesundheitswesen $\underline{66}, 380-386$

Nübling R, Schrempp C, Kress G, Löschmann C, Neubart R, Kuhlmey A (2004b): Qualitätssicherung und Qualitätsmanagement in der stationären Altenpflege. Bundesgesundheitsbla $\underline{47}, 133-140$

Port CL, Zimmerman S, Williams CS, Dobbs D, Preisser JS, Williams SW (2005): Families Filling the Gap: Comparing Family Involvement for Assisted Living and Nursing Home Residents with Dementia. Gerontologist $\underline{45}, 87-95$

Privatinstitut für Evaluation und Qualitätssicherung im Gesundheits- und Sozialwesen $\mathrm{GmbH}$ (eqs-Institut): Bericht zur schriftlichen Befragung der Pflegedienstleitungen zur ärztlichen Versorgung in den Einrichtungen. Manuskript, Hamburg 2008

Przyborski A, Wohlrab-Sahr M: Qualitative Sozialforschung. Ein Arbeitsbuch. 3. Auflage; Oldenbourg Verlag, München 2010 
Rothgang H, Borchert L, Müller R, Unger R: GEK-Pflegereport 2008. Schwerpunktthema: Medizinische Versorgung in Pflegeheimen (Schriftenreihe zur Gesundheitsanalyse Band 66). Asgard-Verlag, Schwäbisch Gmünd 2008

Rurup ML, Onwuteaka-Philipsen BD, Pasman HR, Ribbe MW, van der Wal G (2006):

Attitudes of physicians, nurses and relatives towards end-of-life decisions concerning nursing home patients with dementia. Patient Educ Couns $\underline{61}, 372-380$

Ryan A, Scullion HF (2000): Family and staff perceptions of the role of families in nursing homes. J Adv Nurs $\underline{32}, 626-634$

Sandberg J, Lundh U, Nolan MR (2001): Placing a spouse in a care home: the importance of keeping. J Clin Nurs $\underline{10}$, 406-416

Schäufele M, Köhler L, Lode S, Weyerer S: Menschen mit Demenz in stationären Pflegeeinrichtungen: aktuelle Lebens- und Versorgungssituation. In: Schneekloth U, Wahl HW (Hrsg.): Integrierter Abschlussbericht. Möglichkeiten und Grenzen selbstständiger Lebensführung in stationären Einrichtungen (MuG IV) - Demenz, Angehörige und Freiwillige, Versorgungssituation, sowie Beispielen für „Good Practice“. o.Verl., München 2007, 169-231

Schneekloth U, Von Törne I: Entwicklungstrends in der stationären Versorgung Ergebnisse der Infratest-Repräsentativerhebung. In: Schneekloth U, Wahl HW (Hrsg.): Integrierter Abschlussbericht. Möglichkeiten und Grenzen selbstständiger Lebensführung in stationären Einrichtungen (MuG IV) - Demenz, Angehörige und Freiwillige, Versorgungssituation, sowie Beispielen für „Good Practice“. o.Verl., München 2007, 53-168

Schneekloth U, Wahl HW (Hrsg.): Integrierter Abschlussbericht. Möglichkeiten und Grenzen selbstständiger Lebensführung in stationären Einrichtungen (MuG IV) Demenz, Angehörige und Freiwillige, Versorgungssituation, sowie Beispielen für „Good Practice“. o.Verl., München 2007

Schwartz AN, Vogel ME (1990): Nursing Home Staff and Residents' Families Role Expectations. Gerontologist 30, 49-53

Seidel J, Kelle U: Different functions of coding in the analysis of textual data. In: Kelle U (Hrsg.): Computer-aided qualitative data analysis. Theory, Methods and Practice. Sage Publications, London 1995, 52-61

Shield RR, Wetle T, Teno J, Miller SC, Welch L (2005): Physicians "Missing in Action": Family Perspectives on Physician and Staffing Problems in End-of-Life Care in the Nursing Home. J Am Geriatr Soc 53, 1651-1657

Shuttlesworth GE, Rubin A, Duffy M (1982): Families Versus Institutions: Incongruent Role Expectations in the Nursing Home. Gerontologist 22, 200-208

Sloane PD, Zimmerman S, Williams CS, Hanson LC (2008): Dying With Dementia in Long-Term Care. Gerontologist $\underline{48}, 741-751$ 
Strauss A, Corbin J: Grounded Theory: Grundlagen Qualitativer Sozialforschung. Beltz Psychologie Verlags Union, Weinheim 1996

Struppek D: Patientensouveränität im Pflegeheim: Möglichkeiten und Grenzen aus der Sicht von hochaltrigen, mehrfach erkrankten Pflegeheimbewohnern, ihren Ärzten, Pflegekräften und privaten Bezugspersonen. Phil. Diss. Berlin 2010

Teno JM, Casey VA, Welch LC, Edgman-Levitan S (2001): Patient-Focused, FamilyCentered End-of-Life Medical Care: Views of the Guidelines and Bereaved Family Members. J Pain Symptom Manage 22, 738-751

Teno JM, Clarridge BR, Casey V, Welch LC, Wetle T, Shield R, Mor V (2004): Familie Perspectives on End-of-Life Care at the Last Place of Care. JAMA 291, 88-93

Tsai HH, Tsai YF (2012): Family members' perceived meaning of visiting nursing home residents in Taiwan. J Adv Nurs $\underline{68}, 302-311$

van den Bussche H, Weyerer S, Schäufele M, Lübke N, Schröfel SC, Dietsche S (2009a): Die ärztliche Versorgung von Pflegeheimbewohnern in Deutschland - Eine kritische Würdigung der vorliegenden Studien. Z Allgemeinmed 85, 240-246

van den Bussche H, Schröfel SC, Löschmann C, Lübke N (2009b): Organisationsformen der hausärztlichen Versorgung von Pflegeheimbewohnern in Deutschland und im benachbarten Ausland. Z Allgemeinmed 도, 296-301

Vinsnes AG, Nakrem S, Harkless GE, Seim A (2012): Quality of care in Norwegian nursing homes - typology of family perceptions. J Clin Nurs $\underline{21}, 243-254$

Wetle T, Shield R, Teno J, Miller SC, Welch L (2005): Family Perspectives on End-of-Life Care: Experiences in Nursing Homes. Gerontologist 45, 642-650

Wingenfeld K: Studien zur Nutzerperspektive in der Pflege (Veröffentlichungsreihe des Instituts für Pflegewissenschaft an der Universität Bielefeld P03-124). o.Verl., Bielefeld 2003

Wollny A, Marx G (2009): Qualitative Sozialforschung - Ausgangspunkte und Ansätze für eine forschende Allgemeinmedizin. Z Allgemeinmed $\underline{85}, 467-476$

\subsection{Internetquellen}

AOK Baden-Württemberg: Integrierte Versorgung Pflegeheim (IVP). Vorstellung des Vertrages. Stuttgart 2013

http://www.aok-gesundheitspartner.de/imperia/md/gpp/bw/arztundpraxis/facharzt vertrag/bw_iv_pflegeheim_schulungsfolien_210613.pdf; Zugriff am 25.03.2016

AOK Nordost: Arzt-im-Pflegeheim-Programm. Careplus- das bedeutet eine verbesserte Zusammenarbeit zwischen Pflegekräften, Ärzten und Therapeuten. Potsdam 2015 http://www.aok-gesundheitspartner.de/imperia/md/gpp/bln/pflege/stationaer/care plus/careplus_kurzpraesentation_2015.pdf; Zugriff am 25.03.2016 
AOK-Bundesverband GbR: Verträge zur Integrierten Versorgung (§ 140 a-d SGB V). Berlin o.J.

http://www.aok-gesundheitspartner.de/bund/arztundpraxis/vertraege/index _09729.html; Zugriff am 25.03.2016

Bundesministerium für Justiz und Verbraucherschutz: Heimgesetz in der Fassung der Bekanntmachung vom 5. November 2001 (BGBI. I S. 2970), das zuletzt durch Artikel 3 Satz 2 des Gesetzes vom 29. Juli 2009 (BGBI. I S. 2319) geändert worden ist. Berlin 2009

http://www.gesetze-im-internet.de/bundesrecht/heimg/gesamt.pdf; Zugriff am 25.03.2016

Bundesministerium der Justiz und für Verbraucherschutz: Betreuungsrecht. Mit ausführlichen Informationen zur Vorsorgevollmacht. Berlin 2015 http://www.bmjv.de/SharedDocs/Publikationen/DE/FokusKarussell/Betreuungsrecht.p df?_blob=publicationFile\&v=2; Zugriff am 25.03.2016

Bundesministerium für Justiz und Verbraucherschutz: Das Fünfte Buch Sozialgesetzbuch (SGB) - Gesetzliche Krankenversicherung - (Artikel 1 des Gesetzes v. 20. Dezember 1988, BGBI. I S. 2477, 2482), das zuletzt durch Artikel 2 Absatz 12 des Gesetzes vom 17. Februar 2016 (BGBI. I S. 203) geändert worden ist. Berlin 2016 http://www.gesetze-im-internet.de/sgb_5/BJNR024820988.html\#BJNR02482098 8BJNG008301308; Zugriff am 25.03.2016

Compass Private Pflegeberatung: COMPASS-Befragung. Befragungsergebnisse Pflegeberatung und Belastung Angehöriger. Grafikband. Köln 2015 http://www.compass-pflegeberatung.de/fileadmin/user_upload/Kurzauswertung _Forsa-Befragung_20150715.pdf; Zugriff am 25.03.2016

Internetredaktion des Bundesministeriums für Familie, Senioren, Frauen und Jugend: Erster Bericht des Bundesministeriums für Familie, Senioren, Frauen und Jugend über die Situation der Heime und die Betreuung der Bewohnerinnen und Bewohner. Berlin 2006 http://www.bmfsfj.de/doku/Publikationen/heimbericht/01-Redaktion/PDF-Anlagen/ gesamtdokument,property\%3Dpdf,bereich\%3Dheimbericht,sprache\%3Dde,rwb\%3Dtr ue.pdf; Zugriff am 25.03.2016

Kassenärztliche Vereinigung Mecklenburg-Vorpommern, AOK Nordost: Gemeinsame Medieninformation Kassenärztliche Vereinigung Mecklenburg-Vorpommern AOK Nordost. Erfolgsmodell "Pflegeheim Plus" 50. Pflegeheim in fester Betreuung eines Hausarztteams. Schwerin 2011 http://www.kvmv.info/aerzte/Archiv/Presseinformationen/Presseinformationen_2011/ GemeinsameMedieninformation_PflegeheimPLUS_27092011.pdf; Zugriff am 25.03.2016 
Kassenärztliche Vereinigung Westfalen-Lippe, AOK Nordwest, BKK-Landesverband Nordwest, IKK classic, Sozialversicherung für Landwirtschaft, Forsten und Gartenbau, Knappschaft, Ersatzkassen: - BARMER GEK, Techniker Krankenkasse, DAK-Gesundheit, Kaufmännische Krankenkasse, Hanseatische Krankenkasse, hkk: Vertrag gemäß § 73a SGBV zur Weiterentwicklung der Strukturen bei der Versorgung von Pflegeheimbewohnern in den Regionen Bünde, Lippe, Marl, Münster und Unna. Dortmund 2014

http://www.aok-gesundheitspartner.de/imperia/md/gpp/wl/iv/vertraege/wl_iv_struk turvertrag_mgepa.pdf; Zugriff am 25.03.2016

Kassenärztlichen Vereinigung Berlin, AOK Nordost- Die Gesundheitskasse, IKK Brandenburg und Berlin, Bahn BKK, der Siemens-Betriebskrankenkasse: BerlinerProjekt- Die Pflege mit dem Plus. Vertrag nach § 73c SGB V zwischen der Kassenärztlichen Vereinigung Berlin (KV Berlin) und der AOK Nordost- Die Gesundheitskasse, der IKK Brandenburg und Berlin, der Bahn BKK, der SiemensBetriebskrankenkasse (SBK) (Krankenkassen). Berlin 2011 https://www.kvberlin.de/20praxis/60vertrag/10vertraege/krankenheim/vertrag_pflegepl us.pdf; Zugriff am 25.03.2016

Medizinischer Dienst des Spitzenverbandes Bund der Krankenkassen e. V. (2012): 3. Bericht des MDS nach § 114a Abs. 6 SGB XI. Qualität in der ambulanten und stationären Pflege. Essen 2012

http://www.mdk.de/media/pdf/MDS_Dritter_Pflege_Qualitaetsbericht_Endfassung.pdf; Zugriff am 25.03.2016

Statistische Ämter des Bundes und der Länder: Demografischer Wandel in Deutschland. Auswirkungen auf Krankenhausbehandlungen und Pflegebedürftige im Bund und in den Ländern. Wiesbaden 2010 https://www.destatis.de/DE/Publikationen/Thematisch/Bevoelkerung/Demografischer Wandel/KrankenhausbehandlungPflegebeduerftige5871102109004.pdf?_blob=publi cationFile; Zugriff am 25.03.2016

Statistisches Bundesamt: Pflegestatistik 2013. Pflege im Rahmen der Pflegeversicherung Deutschlandergebnisse. Wiesbaden 2015 https://www.destatis.de/DE/Publikationen/Thematisch/Gesundheit/Pflege/PflegeDeuts chlandergebnisse5224001139004.pdf?_blob=publicationFile; Zugriff am 25.03.2016 


\section{Danksagung}

Für die freundliche Überlassung des Themas der Dissertation sowie die stetige Unterstützung und konstruktive Betreuung danke ich Frau Prof. Dr. HummersPradier.

Mein besonderer Dank gilt Frau Dr. med. Christiane Müller für Ihre engagierte und intensive Betreuung meiner Arbeit sowie für die vielen unentbehrlichen Anregungen.

Für Ihre Unterstützung auf dem Gebiet der qualitativen Forschung danke ich Frau Prof. Dr. Christina Geister.

Des Weiteren bedanke ich mich bei allen Angehörigen, die sich so freundlich zur Teilnahme an der Studie bereit erklärt haben. 